Elsevier Editorial System(tm) for NeuroImage Manuscript Draft

Manuscript Number: NIMG-17-3264R2

Title: Microstructural Imaging of the Human Brain with A 'Super-Scanner': 10 Key Advantages of Ultra-Strong Gradients for Diffusion MRI

Article Type: SI:Microstructural Imaging

Section/Category: fMRI Acquisition/Physics

Corresponding Author: Professor Derek K. Jones, Ph.D.

Corresponding Author's Institution: Cardiff University

First Author: Derek K Jones

Order of Authors: Derek K Jones; Derek K. Jones, Ph.D.; Daniel C Alexander; Richard Bowtell; Mara Cercignani; Flavio dell'Acqua; Damien M McHugh; Karla L Miller; Marco Palombo; Geoffrey J Parker; Umesh Rudrapatna; Chantal Tax

Abstract: The key component of a microstructural diffusion MRI 'superscanner' is a dedicated high-strength gradient system that enables stronger diffusion weightings per unit time compared to conventional gradient designs. This can, in turn, drastically shorten the time needed for diffusion encoding, increase the signal-to-noise ratio, and facilitate measurements at shorter diffusion times. This review, written from the perspective of the National Facility for In Vivo MR Imaging of Human Tissue Microstructure, an initiative to establish a shared 300 $\mathrm{mT} / \mathrm{m}$-gradient facility amongst the microstructural imaging community, describes ten advantages of ultra-strong gradients for microstructural imaging. Specifically, we will discuss how the increase of the accessible measurement space compared to a lower-gradient systems (in terms of $\Delta$, bvalue, and $\mathrm{TE}$ ) can accelerate developments in the areas of 1) axon diameter distribution mapping; 2) microstructural parameter estimation; 3) mapping micro- vs macroscopic anisotropy features with gradient waveforms beyond a single pair of pulsed-gradients; 4) multi-contrast experiments, e.g. diffusion-relaxometry; 5) tractography and highresolution imaging in vivo and 6) post mortem; 7) diffusion-weighted spectroscopy of metabolites other than water; 8) tumour characterisation; 9) functional diffusion MRI; and 10) quality enhancement of images acquired on lower-gradient systems. We finally discuss practical barriers in the use of ultra-strong gradients, and provide an outlook on the next generation of 'super-scanners'. 


\section{Dear Editor}

Thank you for the feedback on our revised manuscript.

The reviewer comments were as follows (with our response in BLOCK CAPITALS)

Reviewer \#1: The authors have largely addressed my concerns. A few touchups that the authors can do on their own during final preparation of the manuscript.

1) Figures with very small text size in the axis or scale bars: Fig 1,2,3 well.. pretty much all of them have some tiny text that is barely readable when the PDF is zoomed in... Some figures. like Fig. 10 were clearly cut from slides. This does not work well. I can not read the text within the figure even if I zoom the PDF way in...

THANK YOU. WE AGREE AND HAVE REVISED THESE FIGURES. THEY ARE NOW ALL HIGHER RESOLUTION AND THE WRITING IS LEGIBLE.

Reviewer \#3: The revision generally satisfied my concerns. I have just two comments. First, please check figure 1, as it was cropped in the pdf file that I got to review. Second, I wonder about the following difference in terminology in the introduction and whether it was intentionally made different: "improvements in measuring axon diameters" and later "characterisation of tumour cellularity and other microstructural features". Why not use the verb "characterise" in both settings? Finally: Thanks for contributing with a well written paper!

THANK YOU. WE HAVE REVISED THE FIGURES, MADE THEM HIGHER QUALITY - AND MADE THE WRITING LEGIBLE. WE ALSO TOOK ON BOARD THE REVIEWER SUGGESTION AND USED THE WORD 'CHARACTERISE' 


\section{HIGHLIGHTS}

We discuss 10 benefits of ultra-strong gradients for microstructure imaging. Examples include:

Improved estimation of axon diameter in vivo. Better fitting of complex microstructure models Improved characterisation of tumours

Facilitation of multi-contrast MRI experiments 
The reviewer comments were as follows (with our response in BLOCK CAPITALS)

Reviewer \#1: The authors have largely addressed my concerns. A few touchups that the authors can do on their own during final preparation of the manuscript.

1) Figures with very small text size in the axis or scale bars: Fig 1, 2,3 well.. pretty much all of them have some tiny text that is barely readable when the PDF is zoomed in... Some figures. like Fig. 10 were clearly cut from slides. This does not work well. I can not read the text within the figure even if I zoom the PDF way in...

> THANK YOU. WE AGREE AND HAVE REVISED THESE FIGURES. THEY ARE NOW ALL HIGHER RESOLUTION AND THE WRITING IS LEGIBLE.

Reviewer \#3: The revision generally satisfied my concerns. I have just two comments. First, please check figure 1, as it was cropped in the pdf file that I got to review. Second, I wonder about the following difference in terminology in the introduction and whether it was intentionally made different: "improvements in measuring axon diameters" and later "characterisation of tumour cellularity and other microstructural features". Why not use the verb "characterise" in both settings? Finally: Thanks for contributing with a well written paper!

\section{THANK YOU. WE HAVE REVISED THE FIGURES, MADE THEM HIGHER QUALITY - AND MADE THE WRITING LEGIBLE. WE ALSO TOOK ON BOARD THE REVIEWER SUGGESTION AND USED THE WORD 'CHARACTERISE'}




\title{
Microstructural Imaging of the Human Brain with A 'Super-Scanner': 10 Key Advantages of Ultra-Strong Gradients for Diffusion MRI
}

\author{
The PIs and Colleagues of the
}

National Facility for In Vivo MR Imaging of Human Tissue Microstructure

*Jones $\mathrm{DK}^{1,2}$, Alexander $\mathrm{DC}^{3,4}$, Bowtell $\mathrm{R}^{5}$, Cercignani $\mathrm{M}^{6}$, Dell'Acqua $\mathrm{F}^{7}, \mathrm{McHugh}_{\mathrm{DJ}} \mathrm{J}^{8,9}$, Miller KL ${ }^{10}$, Palombo $\mathrm{M}^{3}$, Parker GJM ${ }^{8,9,11}$, Rudrapatna US ${ }^{1}$, Tax $\mathrm{CMW}^{1}$

\section{AFFILIATIONS}

1. Cardiff University Brain Research Imaging Centre (CUBRIC), School of Psychology, Cardiff University, Maindy Road, Cardiff, CF24 4HQ, UK

2. School of Psychology, Faculty of Health Sciences, Australian Catholic University, Melbourne, Victoria 3065, Australia

3. Centre for Medical Image Computing (CMIC), Department of Computer Science, UCL (University College London), Gower Street, London, UK;

4. Clinical Imaging Research Centre, National University of Singapore, Singapore

5. Sir Peter Mansfield Magnetic Resonance Centre, School of Physics and Astronomy, University of Nottingham, University Park, Nottingham, United Kingdom.

6. Department of Psychiatry, Brighton and Sussex Medical School, Brighton, UK.

7. Natbrainlab, Department of Neuroimaging, King's College London, London, SE5 8AF, UK

8. Division of Informatics, Imaging and Data Sciences, The University of Manchester, Manchester, UK.

9. CRUK and EPSRC Cancer Imaging Centre in Cambridge and Manchester, Cambridge and Manchester, UK.

10. Oxford Centre for Functional MRI of the Brain, University of Oxford, Oxford, United Kingdom.

11. Bioxydyn Ltd., Manchester, UK.

*Corresponding author

Email: jonesd27@cf.ac.uk; Phone: +44-29-2087-9412 


\section{ABSTRACT}

The key component of a microstructural diffusion MRI 'super-scanner' is a dedicated high-strength gradient system that enables stronger diffusion weightings per unit time compared to conventional gradient designs. This can, in turn, drastically shorten the time needed for diffusion encoding, increase the signal-tonoise ratio, and facilitate measurements at shorter diffusion times. This review, written from the perspective of the National Facility for In Vivo MR Imaging of Human Tissue Microstructure, an initiative to establish a shared $300 \mathrm{mT} / \mathrm{m}$-gradient facility amongst the microstructural imaging community, describes ten advantages of ultra-strong gradients for microstructural imaging. Specifically, we will discuss how the increase of the accessible measurement space compared to a lower-gradient systems (in terms of $\Delta, b$ value, and TE) can accelerate developments in the areas of 1) axon diameter distribution mapping; 2) microstructural parameter estimation; 3) mapping micro-vs macroscopic anisotropy features with gradient waveforms beyond a single pair of pulsed-gradients; 4) multi-contrast experiments, e.g. diffusionrelaxometry; 5) tractography and high-resolution imaging in vivo and 6) post mortem; 7) diffusion-weighted spectroscopy of metabolites other than water; 8) tumour characterisation; 9) functional diffusion MRI; and 10) quality enhancement of images acquired on lower-gradient systems. We finally discuss practical barriers in the use of ultra-strong gradients, and provide an outlook on the next generation of 'superscanners'.

\section{INTRODUCTION}

This article reviews the benefits of incorporating an ultra-strong gradient system (Setsompop et al. 2013), optimised for diffusion magnetic resonance imaging (dMRI), into an MRI scanner for imaging the human brain. It is written from the perspective of the National Facility for In Vivo MR Imaging of Human Tissue Microstructure, an initiative in the United Kingdom to establish an MRI system with ultra-strong (300 $\mathrm{mT} / \mathrm{m}$ ) gradients as a shared facility amongst the microstructural imaging community. We have selected ten key areas where we believe ultra-strong gradients can advance the field of microstructural imaging, beginning each section with motivation for advancing the field in each area, reviewing what has been achieved to date with more commonly-available gradient amplitudes, and then discussing the potential benefits and opportunities afforded by having access to ultra-strong gradients. In some cases, this is in providing marked improvements to measurements that are already attainable at lower gradient amplitude, and in others, it facilitates new measurements that are simply impractical at lower gradient amplitude. We 
then discuss practical challenges in making use of such as system, including issues of safety and engineering aspects, attempt to highlight which limits are fundamental, and which just require engineering.

The main advantage is, of course, in providing a higher q-value/shorter echo time (TE) for a given b-value, and a higher signal-to-noise ratio (SNR) per unit b-value (see Figure 1a). Shorter diffusion time acquisitions also become more practical as higher $b$-values can be achieved, and a wider range of $b$-values can be maintained across all diffusion times $(\Delta)$ (Figure $1 b$ ).

\section{[FIGURE 1 NEAR HERE]}

FIGURE 1: Increase of the accessible parameter space with ultra-strong gradients. (a) b-value vs minimum achievable echo time (TE) for different maximum gradient amplitudes of $40 \mathrm{mT} / \mathrm{m}, 80 \mathrm{mT} / \mathrm{m}$ and $300 \mathrm{mT} / \mathrm{m}$ (see also Setsompop et al., 2013). For each point, the colour coding gives the achieved $\Delta-\delta / 3$ [ms] (colour coding according to $\Delta$ results in a similar figure with the colourbar ranging from $19 \mathrm{~ms}-97 \mathrm{~ms}$ ). These curves were obtained by simulation on the system with a Stejskal-Tanner EPI sequence, Voxel size $=\left[\begin{array}{ll}2.5 & 2.52 .5\end{array}\right] \mathrm{mm}$, no partial Fourier, GRAPPA =2, Multiband $=1$. (b) PGSE $b$-value (colours) plotted as a function of $G$ and $\triangle$ for $G \max =80$ $\mathrm{mT} / \mathrm{m}$ (left two panels) and $300 \mathrm{mT} / \mathrm{m}$ (right panel). Differences in rise times for Gmax $=80$ and $300 \mathrm{mT} / \mathrm{m}(0.4 \mathrm{~ms}$ and $3.6 \mathrm{~ms}$, respectively) mean that lower $\partial$ values are achievable with $G \mathrm{max}=80 \mathrm{mT} / \mathrm{m}$ (e.g. $\partial=5 \mathrm{~ms}$ as shown in the left-most panel), resulting in shorter and longer achievable $\Delta$, although $b$-values are low (note the difference in colour scale between the $\partial=5 \mathrm{~ms}$ panel and the others). For $\operatorname{Gmax}=80 \mathrm{mT} / \mathrm{m}$ and $\partial=11 \mathrm{~ms}$, the range of $\Delta$ values matches the range available with the shortest $\partial$ possible at $300 \mathrm{mT} / \mathrm{m}$ ( $\partial=8 \mathrm{~ms}$, to the nearest integer). All calculations assume $T E=100 \mathrm{~ms}$.

Making measurements on such kit allows us to develop a truly translational pipeline, i.e., enabling measurements in humans that we could only previously make, in vivo, in animals on preclinical systems. Here, we firstly explore how stronger gradients provide improvements in characterising axon diameter, and microstructural parameters in general. Next we discuss the benefits for getting higher resolution characterisation of brain structure both in vivo and post mortem. We then discuss how, by looking at metabolites other than water, diffusion-weighted spectroscopy can provide deeper insights into tissue microstructure, and how ultra-strong gradients can accelerate developments in this area. While microstructural imaging offers huge potential benefits in a range of diseases, we select as an exemplar application the characterisation of tumours, and explore how stronger gradients may improve characterisation of tumour cellularity and other microstructural features. In addition to characterisation of structure, dMRI has been used to characterise brain function, with the study of rapid temporal changes in the microstructural milieu being an interesting application. Given the disparity of results in the literature on this topic, here we consider how ultra-strong gradients may help to cast further light on the relationship between neural activity and diffusion. 
Despite the many potential advantages of ultra-strong gradients, we recognise that access to ultra-strong gradient systems is not likely to be wide-spread in the immediate future, and so we consider how having a small number of such systems, together with machine learning approaches, might still facilitate deeper insight into tissue microstructure beyond the immediate environs of the scanners themselves. We then discuss practical barriers to making full use of such as system, considering the interplay of hardware and physiological safety limits on deployment of such gradient magnitudes, and consider how some of these issues may be addressed in future generations of 'super-scanners'.

\section{MEASURING AXON DIAMETER}

\section{THE CHALLENGE AND ITS IMPORTANCE}

One of the key features of the diffusion-weighted MR signal is its sensitivity to the size of pores with walls that are either fully restricting, or semi-permeable. The longer the characteristic length scale of the pores, the greater the mean displacement, and the lower the diffusion-weighted signal will be. In the brain, this capability underpins the tantalising prospect of estimating and mapping the distribution of axon diameters in each image voxel, since at least in white matter the axonal walls are the primary geometric structures affecting diffusion in the tissue. The axon diameter distribution is one of the key structural features of tissue related to function, as axon diameter relates directly to transmission speed: larger axons transmit signals more quickly (Hursh 1939; Ritchie, 1995; Richardson, McIntyre, \& Grill, 2000). Differences in axon diameter offer a potential early disease indicator in conjunction with loss of axon density, which can arise in a much wider range of conditions. Finally, measurements of axon diameter have potential utility in brain connectivity mapping as a distinguishing feature of particular fascicles with which to resolve ambiguities, such as crossing versus kissing configurations (Girard et al., 2017; Sherbondy, Rowe, \& Alexander, 2010), and can be combined into the estimation of fibre trajectories, where uniformity of microstructural parameters can be assumed, to provide more robust estimates of connections in approaches such as COMMIT (Daducci, Dal Palù, Lemkaddem, \& Thiran, 2015b).

\section{BACKGROUND}

Several groups of researchers have made attempts to estimate and map the axon diameter distribution in nervous tissue. Stanisz et al. (Stanisz, Szafer, Wright, \& Henkelman, 1997) designed a mathematical model 
of the signal from nerve tissue comprising separate signals from axonal water, glial water, and extra-cellular water, and assumed that there is restricted diffusion in an ellipsoidal geometry in the axonal compartment. They acquired a rich spectroscopic data set from fixed nerve-tissue samples with a wide range of b-values and diffusion times. The parameters of this model that best fit the data provide estimates of the volume fractions and dimensions of the various tissue components - in particular, fitting the model to the data identifies dimensions of the ellipsoidal geometry, the minor axis of which is an estimate of the axon diameter. Assaf et al. (Assaf, Blumenfeld-Katzir, Yovel, \& Basser, 2008) later presented the AxCaliber technique, which uses a similar model to Stanisz, but assumes cylindrical axonal geometry with a gamma distribution of diameters; these authors also simplify the model by assuming no glial cell contribution and no exchange among compartments. They show a similar spectroscopic experiment to Stanisz using two nerve samples (sciatic, which has relatively large axons, and optic, with smaller diameters) and show a good match between the estimated axon diameter distribution (parameters of the fitted gamma distribution) and the histogram of diameters measured on histology of the same samples. Later work by the same group (Barazany, Basser, \& Assaf, 2009) combined AxCaliber with imaging to map the axon diameter distribution over the corpus callosum of a live rat and again show good agreement with histological analysis of the axon diameter distribution in sagittal sections of the mid-line (see Figure 2a).

A key assumption in the techniques above is that the fibre orientation is fixed, and perpendicular to the direction of the applied diffusion encoding gradient. While this condition can be satisfied for limited portions of white matter, extending the approach to the whole brain clearly requires a generalisation of the method, to account for:

(i) different orientations of a single bundle;

(ii) multiple distinct fibre bundles passing through the same voxel; and

(iii) intra-voxel orientational dispersion of a given fibre bundle.

ActiveAx (Alexander et al., 2010) addressed issue (i), by combining high angular resolution diffusion imaging (HARDI) with a simplified model designed to minimise complexity. In this way, they provide an orientationally invariant estimate of a single axon diameter index (approximately a volume-weighted mean (Alexander et al., 2010; Benjamini, Komlosh, Holtzclaw, Nevo, \& Basser, 2016); but also see (Burcaw, Fieremans, \& Novikov, 2015) for a detailed discussion). Experiments with fixed monkey brains recover the low-high-low trend in axon diameter across the mid-sagittal corpus callosum (known from histology to persist across mammalian species (Aboitiz and Montiel, 2003; Olivares et al. 2001) with high reproducibility, and preliminary results from human volunteers show similar trends albeit more weakly (see Figure 2b). AxCaliber3D (Barazany, Jones and Assaf, 2011) addressed issues (i) and (ii) above (i.e., recovering distinct axon diameter distributions for multiple fibres within the same voxel), by acquiring CHARMED (Assaf \& Basser, 2005) data sets at multiple diffusion times. Once the CHARMED model is fitted, it is possible to simulate the diffusion-time dependence of the signal at arbitrary orientations and therefore perpendicular to any fibre component within the voxel. These data are inputted into the AxCaliber 
framework to recover an apparent axon diameter distribution for that fibre population. Refinements to ActiveAx address issues (ii) and (iii): within-voxel fibre crossings (Zhang, Dyrby, \& Alexander, 2011a), as well as within-voxel fibre-orientation dispersion (Zhang, Hubbard, Parker, \& Alexander, 2011b). These advances in modeling extend the portion of white matter over which we can obtain sensible axon-diameter indices. More recent advances in model-based techniques use refined models, which e.g. capture the timedependence of extra-cellular diffusion (De Santis, Jones, \& Roebroeck, 2016; Sepehrband, Alexander, Kurniawan, Reutens, \& Yang, 2016) and non-standard pulse sequences, such as oscillating gradients (Kakkar et al., 2017; Siow, Drobnjak, Chatterjee, Lythgoe, \& Alexander, 2012; Xu et al., 2014; 2016), double diffusion encoding (Benjamini et al., 2016) and generalized waveforms (Drobnjak, Siow, \& Alexander, 2010; Drobnjak, Zhang, Hall, \& Alexander, 2011). Alternative estimation approaches include a q-space formulation (Ong et al., 2008; Ong \& Wehrli, 2010), convex optimization (Daducci, Dal Palù, Lemkaddem, \& Thiran, 2015b), integration with fibre-tracking (Sherbondy, Rowe \& Alexander, 2010; Daducci, Dal Palù, Lemkaddem, \& Thiran, 2015b; Girard et al., 2017) and dictionary matching (Sepehrband et al., 2016).

\section{THE PROMISE}

One of the fundamental limits to axon-diameter estimation is the so-called "resolution limit" of the diffusion experiment. The diffusion-weighted MR signal is sensitive only to a window of restriction lengths and the bounds of that window depend strongly on the available gradient strength. Moreover, most axon diameters lie below the lower bound of the window (the resolution limit) given the available gradient strength in most currently available MRI scanners. Dyrby et al. (Dyrby, Søgaard, Hall, Ptito, \& Alexander, 2012; Dyrby et al., 2012) demonstrate empirically the advantages of increasing gradient strength in axon diameter mapping using simulations and ex vivo measurements on a monkey corpus callosum using a small-bore scanner with $400 \mathrm{mT} / \mathrm{m}$ gradients (see Figure 2c). Drobnjak et al. (Drobnjak, Zhang, lanuş, Kaden, \& Alexander, 2016) identified the resolution limit numerically for combinations of pulsed gradient spin echo (PGSE) and oscillating gradient spin echo (OGSE) measurements, and showed that in the idealised experiment where the gradients are perpendicular to straight, parallel, impermeable cylinders, the standard PGSE experiment gives the greatest sensitivity and that the resolution limit on axon diameter is in the range 4.5-7um (depending on available SNR) with $60 \mathrm{mT} / \mathrm{m}$, but reduces to $2-3$ um with $300 \mathrm{mT} / \mathrm{m}$. In the more realistic situation for brain tissue, where the magnetic field gradient is not quite perpendicular to the fibres and/or the fibres have dispersed orientations, the resolution limit increases, although using OGSE instead of PGSE mitigates this increase to some extent. For example, with the gradient 10 degrees from perpendicular, or with moderate orientational dispersion (characterised by a Watson distribution with a dispersion parameter of 16), using OGSE, the resolution limit increases by about 1um (see Figure $2 \mathrm{~d}$ ).

FIGURE 2 NEAR HERE

FIGURE 2-Various results estimating axon diameter distribution from the literature (see text). 
(a) results from Barazany et al. (2009) comparing electron microscopy data and in vivo estimates of axon diameter distribution in the rat brain; (b) Axon diameter index maps in fixed monkey brains showing a characteristic lowhigh-low trend in axon diameter across the mid-sagittal corpus callosum from Alexander et al 2010; (c) Comparison of axon diameter index maps at increasing (top to bottom) maximum gradient strength using ex vivo measurements on a monkey corpus callosum from a small-bore scanner with $400 \mathrm{mT} / \mathrm{m}$ gradients from Dyrby et al. (2012); (d) demonstration of benefit of higher SNR and gradient amplitude for minimum resolvable diameter ( $y$ axis) (from Nilsson et al. 2017) both with no fibre orientation dispersion (left) and with fibre orientation dispersion (right).

Nilsson et al. (Nilsson, Lasič, Drobnjak, Topgaard, \& Westin, 2017) confirmed these findings analytically. They showed, for perfectly straight parallel cylinders and with the gradient perfectly perpendicular to them, (i.e., with very little attenuation in the intra-axonal water signal), that for a PGSE sequence: $d_{\min }=$ $\left(1 / \sqrt{G_{\max }}\right)\left(1 / \delta^{\frac{1}{4}}\right)\left(\bar{\sigma} D_{0}\right)^{\frac{1}{4}}\left(768 / 7 \gamma^{2}\right)^{\frac{1}{4}}$, where $D_{0}$ is the free diffusivity of the intra-axonal water, $\mathrm{G}_{\max }$ is the maximum gradient amplitude, $\delta$ is their pulsed duration, equal to the diffusion time, $\Delta$, and $\bar{\sigma}$ is the minimal detectable percentage change in the signal when the gradients are applied (i.e., the equation holds at a particular SNR that is assumed unaffected by, e.g., the choice of $\delta$ ). Critically, the resolution limit depends on $\mathrm{G}_{\max }{ }^{-1 / 2}$ as well as $\mathrm{D}_{0}{ }^{1 / 4}$, for any shape of gradient waveform. In addition to strong gradients, this also calls for a high signal to noise ratio, which may be obtained by more efficient readout schemes (such as spiral read-out to shorten the echo time), more sensitive receiver coils, or indeed by increasing the static $\left(B_{0}\right)$ field strength.

The resolution limit raises the question of whether diffusion MRI can provide any useful features of the axon diameter distribution at all, since histological measurements of most axons are 1 um or less - at least a factor of 2 below our best-case resolution limit. However, a few further considerations may renew hope, particularly with the benefits of $300 \mathrm{mT} / \mathrm{m}$ gradient systems. First, histological preparation typically causes tissue shrinkage and in-situ and in vivo axons may be somewhat larger in diameter than histological measurements suggest. The degree of shrinkage is hard to establish, but estimates range between 0-65\% (Alexander, Dyrby, Nilsson, \& Zhang, 2017a). Second, although large axons are a tiny minority in terms of number of axons, they are much more significant in terms of the MRI signal they contribute, because of the relative volume of water they contain. This means that variation in the large-diameter tail of the axon diameter distribution has a disproportionate influence on the MR signal contrast. Figure 3 highlights the practical importance of this observation - although less than $10 \%$ of the axons in this example have diameter over 3um, those axons provide $50 \%$ of the intra-axonal signal. Moreover, as the resolution limit decreases (due to gradient amplitude increasing from $60 \mathrm{mT} / \mathrm{m}$ to $300 \mathrm{mT} / \mathrm{m}$ ), the proportion of axons above the resolution limit changes slowly from about $5 \%$ to about $20 \%$. However, the proportion of the signal that comes from these axons increases much more - from about 35\% to over $70 \%$ for the same increase in gradient strength. This suggests that diffusion MR experiments may hold strong sensitivity at 
least to variability in the existence and size of large axons. It further highlights that $300 \mathrm{mT} / \mathrm{m}$ gradients offer a major benefit in recovering information from the axon diameter distribution; early results in the brain (with and without multiple sclerosis pathology) (Huang et al., 2015; Huang et al., 2016) and in the spinal cord (Duval et al., 2017) show promise in exploiting these advantages. Given the fact that there is a differential sensitivity of axons, according their diameter, in diseases such as multiple sclerosis (DeLuca et al., 2004), the potential gains from being able to map apparent axon diameter indices are clear.

\section{FIGURE 3 NEAR HERE}

Figure 3. Illustration of the benefit of gradient strength on recovering features of the axon diameter distribution. Panel (a) shows a histogram of axon diameters from the corpus callosum of a post-mortem human brain, compiled from the data in (Aboitiz, Scheibel, Fisher, \& Zaidel, 1992), scaled by the inverse of a typical shrinkage factor of 1.5. The red curve is the cumulative distribution. Panel (b) shows the same histogram as in (a) but now weighted by axonal volume - the histogram of space occupied by axons of each diameter, which corresponds to the proportion of signal contributed by axons in each size bin; again the red line is the cumulative distribution. In panel (c), the graph shows the proportion of axons (in blue) that are above the resolution limit, as a function of available gradient strength, as well as the proportion of intra-axonal signal (in red) that comes from such axons. Error bars show how that proportion varies for histological shrinkage factors over the range: $[1,2]$. The simulation accounts for the limitations on rise time imposed by physiological safety constraints (see Section 11).

The resolution limit is not the only challenge for axon diameter mapping. Major challenges remain in identifying models that link the MR signal to underlying tissue parameters sufficiently well to isolate contrast specifically arising from the axon diameter distribution. A range of other effects can confound the estimation. For example, although the original work on ActiveAx (Alexander et al., 2010) showed trends in axon diameter consistent with expectations from histology (Aboitiz et al., 1992), there was nevertheless a substantial overestimation of the axon diameter index in both in vivo and ex vivo experiments. The authors suggest orientation dispersion as one possible explanation. However, even when accounting for orientational dispersion (Zhang et al., 2011b) there is still substantial, albeit reduced, overestimation. This could, in part, be attributable to the definition of 'orientational dispersion': Zhang et al. (Zhang et al., 2011b) primarily consider only macroscopic dispersion, i.e., variation in orientation among axons, each of which is assumed to be straight. However, microscopic orientation dispersion, where individual axons vary in orientation over the extent of an image voxel (e.g., axonal undulation), can have a similar confounding effect leading to the characteristic length scale being driven by the amplitude of the undulation as well as axon diameter (Nilsson, Lätt, Ståhlberg, van Westen, \& Hagslätt, 2012). Exchange between the intra-axonal and extracellular space due to non-zero cell membrane permeability can also potentially lead to overestimation. While such exchange can be incorporated into the modeling process as a parameter of 
interest in its own right, see e.g. (Nedjati-Gilani et al 2017), strong gradients resulting in shorter echo times can alternatively help to reduce the influence of exchange. Other effects such as complexity of the extracellular diffusion process (Burcaw et al., 2015) and differences in the relaxation parameters of different water pool , (see Section 4), can also affect the signal significantly, but are often ignored in models used for axon diameter estimation; a study by De Santis et al. (De Santis et al., 2016c) reports a reduction of estimation bias in axon diameter estimates when modeling time-dependence of the extra-axonal diffusivity.

Despite this bias in estimates of features of the axon diameter, Horowitz et al. (Horowitz et al., 2014) showed correlation between estimates of apparent axon diameter distribution and inter-hemispheric apparent conduction velocity, inferred from electrophysiological measurements, suggesting that these measurements might explain individual differences in brain function. This result suggests that even with a lack of accuracy in characterising the absolute axonal distribution it may be possible to define useful imaging biomarkers that characterise disease and relate strongly to axon diameter. Criticism of the work (Innocenti, Caminiti, \& Aboitiz, 2015) focuses on the resolution limit and potential bias in estimates of the axon diameter distribution from diffusion MRI. Of course, if the bias (e.g., over-estimation) in axon diameter estimate is approximately uniform, or even monotonic, sufficient correlation may remain to infer apparent conduction velocity from axon diameter distribution estimates. Whether we can predict actual conduction velocity from microstructural measurements in vivo is an altogether different question.

In summary, although it is a compelling application of diffusion MRI, major challenges remain in obtaining trusted measurements and maps of the axon diameter distribution from MRI. However, even having access to apparent axon diameter distributions might give enhanced sensitivity to white matter disease, earlier response to intervention, and increased prognostic value, when compared to other techniques that ignore differences in axon diameter, or treat them as invariant. The range of confounding effects mean that we are yet to be certain about the exact sensitivity to axon diameter distributions, or the range of effects to include in models that enables us to isolate that sensitivity. Nevertheless, in the words of Nilsson et al (Nilsson et al., 2017): “... the diffraction limit in optical microscopy, introduced in 1873, was recently broken. It took 135 years. Perhaps breaking the resolution limit in diffusion MRI can be done a little faster?"

\section{PARAMETER ESTIMATION}

\section{THE CHALLENGE AND ITS IMPORTANCE}


To describe the complex diffusion process in tissue, a variety of approaches have been proposed that relate the signal to diffusion features or tissue microstructure, of which some have been described in the previous section (see: Panagiotaki et al., 2012; Assemlal et al., 2011; Tournier et al., 2011; and Novikov et al. 2018) for further discussion). The subsequent step of 'fitting' such models or representations to the dMRI data brings up additional challenges. In this process, one tries to find the values of the model parameters that could have generated the observed diffusion weighted signals. Solving this 'inverse problem' generally involves the estimation of more than one free parameter. It is the considerable number of parameters associated with most dMRI models, together with the typically low SNR in diffusion MRI, that make robust microstructural parameter estimation quite challenging. Moreover, the non-Gaussian distribution of the RF noise in the dMRI signal (Aja-Fernandez 2016), as well as measurement errors from further signal perturbations such as motion and distortion (Le Bihan et al. 2006; Pierpaoli 2010; Tax et al. 2016), further complicate the recovery of accurate and precise microstructural estimates from models of tissue microstructure. Even in the context of diffusion tensor MRI (DT-MRI) (Basser \& Jones, 2002), with its relatively 'conventional' b-value (e.g., b $1000 \mathrm{~s} / \mathrm{mm}^{2}$ ) acquisition, parameter estimation is not straightforward: there is a vast body of literature focusing on DT-MRI estimation procedures such as leastsquares (Koay, Carew, Alexander, Basser, \& Meyerand, 2006; Veraart et al., 2012; Veraart, Sijbers, Sunaert, Leemans, \& Jeurissen, 2013) (linear, weighted linear, nonlinear) and maximum likelihood (Fillard, Pennec, Arsigny, \& Ayache, 2007; Landman, Bazin, \& Prince, 2007; Liu, Gasbarra, \& Railavo, 2016); as well as on the incorporation of different constraints, regularizations, re-parameterizations, and outlier rejection strategies(Chang et al. 2005; Chang et al. 2012; Parker et al. 2013; Collier et al. 2015; Tax et al. 2015; Parker and Jones 2017)

For the parameter estimation of multi-compartment biophysical models, non-linear estimation procedures are commonly employed. Nonlinear fitting methods try to find an optimum by gradually moving around in a 'nonlinear parameter landscape', which is time-consuming and prone to getting stuck in local optima.

\section{BACKGROUND}

To address some of the issues in nonlinear fitting on currently available data, the parameter search space can be reduced by decoupling the estimation of the number and orientation of fascicles, from the microstructural parameters of each fascicle. Daducci et al. (Daducci, Canales-Rodríguez, Zhang, Dyrby, Alexander, et al., 2015a) proposed the AMICO framework that linearizes the problem and drastically speeds up the fitting.

Alternatively, the fitting of multi-compartment parameters can be facilitated by first obtaining some main characteristics of the signal. For example, a rotationally invariant representation of the signal can be derived prior to fitting, e.g. the powder average (also called the orientational average, spherical mean, 
directional mean, isotropic mean) (Topgaard and Söderman 2002; Kaden, Kruggel, \& Alexander, 2016; Jespersen, Lundell, Sønderby, \& Dyrby, 2013; Lasič, Szczepankiewicz, Eriksson, Nilsson, \& Topgaard, 2014), possibly accompanied by higher order invariants (Mirzaalian et al. 2015; Novikov, Veraart, Jelescu \& Fieremans, 2018). From this rotationally-invariant signal, the voxel-averaged microscopic properties can subsequently be derived.

Fieremans et al. (Fieremans, Jensen, \& Helpern, 2011) first fit the DKI representation and derive microstructural parameters through analytical relationships with kurtosis parameters. Fick et al. (Fick, Wassermann, Caruyer, \& Deriche, 2016) first fit a regularized version of the mean apparent propagator (MAP) method (Özarslan et al. 2013) and then extrapolate the signal as a pre-processing step for multicompartment model fitting. The advantage is that the multi-compartment estimation can benefit from advanced estimation methods that have already been developed for these other techniques, in addition to the inherent regularisation they provide. Different configurations of multi-compartment models have been evaluated in preclinical acquisitions (Panagiotaki et al., 2012) and in in vivo human studies (Bourne et al., 2014;Ferizi et al., 2014). In group studies, multi-compartment models have revealed microstructural changes in a range of neurological and psychiatric disorders, e.g. (De Santis et al., 2017; Kamagata et al., 2017; Schneider et al., 2014; Slattery et al., 2017);

Despite recent efforts in parameter estimation, it has become increasingly apparent that the ultimate aim of being unambiguously specific to the relevant microstructural characteristics can hardly be achieved with the gradient hardware currently available on clinical MRI systems. Fixing certain microstructural model parameters to a priori values that are assumed to be accurate for the living human brain is a common approach to alleviating the problems with non-linear fitting. However, fixing parameters can generate bias in the other estimated parameters, calling into question their 'true specificity' to the biological reality (Jelescu, Veraart, Fieremans, \& Novikov, 2016).

The parameter landscape and fitting has been extensively studied for an often used multicompartment dMRI model - sometimes referred to as the 'standard model' - in which each compartment can be described by an anisotropic Gaussian function (i.e. a tensor) (Kroenke et al., 2004; Jespersen et al, 2007,2010), an extension of the model developed by Stanisz et al (Stanisz et al., 1997) and refined by Assaf et al. (Assaf \& Basser, 2005). In this model, the measurement is assumed to be beyond the resolution limit described in Section 1 and as such the intra-neurite space is represented as sticks (tensors with zero perpendicular diffusivity). The compartment configuration where the intra-neurite space has perpendicular diffusivity equal to zero, has been used in various approaches such as NODDI (Zhang, Schneider, WheelerKingshott, \& Alexander, 2012) and the ball and stick model (Behrens et al., 2003). The extra-neurite space is described by a prolate tensor with nonzero parallel- and perpendicular diffusivities. For reliable fitting of this model, it is often assumed that parameters in the model exhibit the same value (e.g., the parallel intra- 
and extra-neurite diffusivities are the same), or are fixed to some known value. In the work of Jelescu et al. (Jelescu et al., 2016) all parameters were allowed to be independent of each other. It was found empirically (Jelescu et al., 2016), and analytically (Novikov, Veraart, Jelescu \& Fieremans, 2017), that the optimization landscape is very flat, and that the fitting is intrinsically degenerate with two solution 'branches' that are equally biologically plausible: parallel intra-neurite diffusivity is larger than parallel extra-neurite diffusivity, or vice versa. For acquisitions up to typical 'clinical' b-values ( $2500 \mathrm{~s} / \mathrm{mm}^{2}$ ), it was shown that it impossible to determine which branch is correct, even in the simplest case of straight parallel fibres. Experiments without fixed parameters (Lampinen et al. 2017) indicate that the assumption of equal parallel diffusivities does not seem to hold in WM; neither does the widely employed tortuosity assumption .

\section{THE PROMISE}

For the 'standard' model described above, high b-value measurements with sufficient SNR hold the potential to determine which is the correct solution branch to adopt, allowing estimation of all the parameters unambiguously, without having to fix them a priori (Jelescu et al., 2016). Preliminary results with b-values up to $10,000 \mathrm{~s} / \mathrm{mm} 2$ from an $80 \mathrm{mT} / \mathrm{m}$ gradient system conjecture that the parallel intraneurite diffusivity is larger in WM, but noise still challenges the estimation and branch selection remains an open question.. Compared to standard hardware, ultra-strong gradients allow an increased SNR per unit time can be obtained for high b-values, so that the high b-value parameter space can be studied into more detail and the findings at lower gradient strengths can be validated.

In the case of ambiguities / degeneracies, i.e., where different microstructural configurations lead to the same diffusion-weighted signal (such as the solution 'branches 'discussed above), 'orthogonal' measures that bring new information to the problem are crucial (Novikov et al. 2016; Lampinen et al. 2017). The potential widespred benefit of the super-scanner, therefore, is to provide measurements that are not normally available, but which can inform on parameters in the measurements that we can normally obtain. This provides a more informed strategy for choosing fixed values or priors to stabilize models we fit to routinely-acquired data.

\section{MACROSTRUCTURAL vs MICROSTRUCTURAL ANISOTROPY}


The majority of approaches developed to extract diffusion- or microstructural features from dMRI data, are built on the Stejskal-Tanner sequence (Stejskal \& Tanner, 1965) (or 'single diffusion encoding', SDE). Diffusion tensor MRI (Basser, Mattiello, \& LeBihan, 1994), still being the most commonly applied SDE-based method to showcase sensitivity of dMRI to pathology, merely provides information on the average diffusion process within a voxel. Macroscopic DTI features such as fractional anisotropy (FA) and mean diffusivity (MD) concomitantly give an indication of the average orientation, shape, and size of the diffusion process on the voxel-scale. In reality, however, tissue is more accurately described as being composed of different compartments with distinct characteristics: a distribution of microenvironments (represented by a sum of tensors under certain assumptions (Szczepankiewicz et al., 2015)). See Figure 4a

FIGURE 4a,b NEAR HERE

Figure 4.

a. The difference between microscopic and macroscopic anisotropy, and impact of size variance and orientation coherence on macroscopic anisotropy. $V_{I}=$ isotropic volume fraction; $V_{A}=$ anisotropic volume fraction; $F A=$ (macroscopic) fractional anisotropy; $\mu F A=$ microscopic fractional anisotropy. Despite having the same $\mu F A$, the different substrates in the right hand column (for example) have difference macroscopic FA values, purely due to orientational dispersion. (Taken from the Ph.D. thesis of Filip Szczepankiewicz, Lund University, with permission) b. Comparison of $\mu F A$ and FA in the same healthy (age $=45$ years) participant. Note the areas of low FA (dark) where different pathways cross / merge, while the $\mu F A$ is relatively uniform in white matter.

Consequently, the macroscopic FA cannot provide an interpretable marker other than a vague concept of 'tissue integrity' (Jones, Knösche, \& Turner, 2013); the voxel-scale diffusion tensor entangles the information about the orientation, shape, and size of the microenvironments. It can therefore not distinguish between different configurations of orientation incoherence, microscopic anisotropy, and isotropic heterogeneity.

Many alternative strategies, based on SDE, have been proposed that aim to resolve the limitations of DTI and to distinguish between different configurations. Orientation incoherence can for example be accounted for by explicitly modeling crossing or dispersing microenvironments as for example in spherical deconvolution-based frameworks (e.g.Tournier et al. 2004, 2007; Jeurissen et al. 2014), Lätt et al. 2003) or in NODDI (Zhang et al. 2012), or by factoring out the orientational dependency (Basser \& Pierpaoli, 1998; Edén, 2003; Kaden et al., 2016a, 2016b). However, a common underlying assumption is that the kernel (or response function, or single-fiber profile (Tax, Jeurissen, Vos, Viergever, \& Leemans, 2014)) is the same along every orientation. Obtaining such information on the average shapes and sizes of the microenvironments that constitute the kernel has already turned out to be challenging based on SDE measurements alone (e.g., an intra- and extra-neurite compartment) - as discussed in Section 2. In 
addition, the assumption of a single kernel per voxel may not always hold, e.g. in the case of pathology affecting one of multiple crossing fascicles.

SDE-based approaches that aim at quantifying the heterogeneity of diffusivities include statistical approaches (Yablonskiy \& Sukstanskii, 2010), q-space approaches (King et al. 1994; Assaf and Cohen 2000; Cohen and Assaf 2002; Assaf et al. 2002; Lätt et al. 2008), and diffusion kurtosis imaging (Jensen, 2015; Szczepankiewicz et al., 2015). Statistical approaches, for example, describe the signal as a sum of signals from spin packets that originate from different positions within a voxel (Scherrer et al., 2016; Yablonskiy \& Sukstanskii, 2010). These spin packets interfere with different microenvironments and their contribution to the total signal can be described as a distribution of apparent diffusivities. However, if a voxel with a low macroscopic fractional anisotropy has a broad distribution of apparent diffusivities, it is impossible to tell if this is due to multiple isotropic compartments with variable diffusivity (isotropic heterogeneity), or due to multiple anisotropic compartments with variable orientation (microscopic anisotropy and orientation incoherence), or both.

Taken together, while many strategies have been proposed to overcome the shortcomings of DTI, SDEbased techniques are inherently limited in that they cannot disentangle variations in size, shape, and orientation without prior knowledge or assumptions about the compartmentalisation, that have to be explicitly incorporated as constraints in signal modeling. Alternative acquisition strategies beyond SDE are essential to get a more complete picture of the distribution of microenvironments in the tissue.

\section{BACKGROUND}

Instead of using a single pair of diffusion pulses to encode the signal as in SDE, multiple periods of diffusion encoding can be employed to establish displacement correlations of spins. Double diffusion encoding (DDE, (Mitra, 1995; Cory, Garroway, Miller, 1990; Shemesh and Cohen 2008) and multiple diffusion encoding (MDE) sequences have two or more diffusion encoding periods possibly at an angle ('angular DDE'), and can distinguish the scenarios of spherical compartments from incoherently oriented anisotropic compartments while relaxing modeling constraints (Lawrenz \& Finsterbusch, 2013). As such, it becomes possible to derive microscopic anisotropy measures like the micro-FA that, in analogy to FA (or macro-FA) is related to the variance of the eigenvalues of the microenvironment diffusion tensors. In the absence of orientation incoherence, the micro-FA and macro-FA are the same. DDE and MDE measurements are ideally designed to be rotationally invariant (Jespersen et al., 2013), so that the measured micro-FA does not depend on the orientational configuration of the tissue under investigation.

In addition to pulsed field gradients, more arbitrary time varying gradient waveforms have been proposed (Drobnjak \& Alexander, 2011; Lasič et al., 2014; Lundell et al. 2015; Westin et al., 2016) that can have a 
higher encoding efficiency than DDE to facilitate imaging in a clinical setting. The general time-varying waveforms describe a trajectory in q-space, referred to as Q-space trajectory encoding (QTE) (Westin et al., 2016). In this framework, the q-space trajectory can be characterised by its B-tensor (or second-order moment). The B-tensor is a natural extension of the $b$-value (with the b-value being its trace) and has previously been adopted to take into account cross-terms related to the imaging gradients (Karlicek \& Lowe, 1980; Mattiello, Basser, \& Le Bihan, 1997; Mattiello, Basser, \& LeBihan, 1994). SDE and angular DDE acquisitions can in this framework be described by 'linear' B-tensors of rank 1 and 'planar' B-tensors of rank 2, respectively. 'Spherical' B-tensors (or 'isotropic encodings') have been used to obtain fast trace-weighted images (Mori \& van Zijl, 1995; Eriksson, Lasič, \& Topgaard, 2013; Wong, Cox, \& Song, 1995).

Microscopic diffusion anisotropy has been measured with clinical-gradient systems using DDE (Hui \& Jensen, 2015; Lawrenz, Brassen, \& Finsterbusch, 2016) and QTE (Lasič et al., 2014). Several analysis frameworks have been developed that aim to extract additional features from measurements beyond SDE (Hui \& Jensen, 2015; Jespersen et al., 2013; Westin et al., 2016) on clinical systems. If it is assumed that the tissue can be represented by a distribution of diffusion tensors, then, by exploiting the cumulant expansion of the QTE signal (Westin et al., 2016), information on the covariance of the microenvironment tensors can be obtained. Significant differences in such features were found between a small population of healthy controls and schizophrenia patients. Alternatively, the DIVIDE framework (Szczepankiewicz et al., 2015) uses a parameterisation of the distribution (e.g. a Gamma distribution) and relates this to the powderaveraged signal obtained from varying B-tensor shapes. In this way, isotropic heterogeneity and microscopic anisotropy could be disentangled and the isotropic variance component could be studied in vivo. This approach has been used to study the heterogeneity in meningiomas and gliomas, and it was shown that the dominant type of variance (isotropic or anisotropic) was different in both tumours, and that this correlated with microscopy findings (Szczepankiewicz et al. 2016).

Measurements beyond SDE have also been used in conjunction with biophysical tissue models that had originally been developed and evaluated based on SDE measurements. DDE and QTE measurements provide invaluable additional information to validate commonly adopted constraints (Lampinen et al, 2017) and to substantiate which compartments to include (Benjamini \& Basser, 2016). Spherical tensor encoding has been used to investigate whether a still water compartment (or 'dot' compartment), which could represent relatively immobile water in small spherical structures such as cells, indeed explains the better fit in WM previously observed with SDE data.

\section{THE PROMISE}


Methods that extract features of the distribution of tensors (assuming to represent the distribution of microenvironments) in the in vivo human brain commonly make some assumptions about the shape of the distribution. In physical chemistry, unconstrained inversion is a method that is commonly employed to find solutions for the distribution of microenvironments in a variety of samples (Kim, Doyle, Wisnowski, Kim, \& Haldar, 2017; Veraart, Novikov, \& Fieremans, 2017), e.g. liquid crystals or rock samples. However, these methods are generally ill-conditioned and very sensitive to noise, and require the signal to be sampled over a wide range of b-values with sufficient SNR. On clinical systems with $40 \mathrm{mT} / \mathrm{m}$ gradients, performing an unconstrained inversion to obtain solutions for the full tensor distribution is therefore likely less stable.

Using ultra-strong gradients, it becomes feasible to access a larger part of the b-value space. Preliminary results (Tax et al., ISMRM 2018) show that unconstrained inversion yields results in agreement with known anatomy. Even though non-standard encoding with strong gradients raises challenges (e.g. gradient nonlinearities, Section 11), it is without question that with ongoing developments to better understand and reduce the influence of such confounds, the fidelity of the inversion will be improved by the increased SNR and larger b-value range that strong gradients provide. Arbitrary waveforms can be combined with hybrid experiments (Section 4) to obtain a comprehensive description of the chemical composition, density, size, shape, and orientation of in vivo human brain tissue. The unconstrained inversion can be used to explore healthy and pathological tissues in which it is unclear which constituents are present and to find suitable constraints.

DDE and arbitrary waveforms often require a relatively long TE. Ultra-strong gradients in combination with optimised waveforms (Drobnjak et al. 2010; Drobnjak et al. 2011; Sjolund et al. 2015) can significantly reduce the TE in such experiments. For example, the signal fraction of a still water compartment could be underestimated at the long TE obtainable on clinical systems if its T2 is shorter than that of other compartments (Veraart et al., 2017; Wilm et al., 2017). Sampling the signal at shorter TE allows to investigate this possible confound. Moreover, the shorter TE afforded by the stronger gradients results in an improvement in SNR of approximately 50\% compared to that achievable on a system with $80 \mathrm{mT} / \mathrm{m}$ gradients.

\section{MULTI-CONTRAST EXPERIMENTS}




\section{THE CHALLENGE AND ITS IMPORTANCE}

A variety of MRI contrasts have been used to characterize tissue beyond dMRI, such as relaxometry, susceptibility, and magnetization transfer. Multi-compartment models have become particularly popular in the attempt to provide a suitable description for each of these contrasts. Most commonly, such multicompartment models are fitted to the signal from each MRI modality separately, and the joint and complementary information between contrasts therefore remains unexploited. In addition, important challenges in the multi-compartment modeling of each of the contrasts hampers an unequivocal characterization of the tissue microstructure.

There are challenges that are inherent to the mathematical formulation of these models, however: in dMRI and relaxometry, for example, the signal attenuation arising from the putative compartments is commonly described by a sum of exponentials. In Section 2, fitting challenges in dMRI were already highlighted for the case of a commonly used model with Gaussian compartments. The mathematical challenge of decomposing the multi-exponential decay into its constituents in the presence of noise is a fundamental problem that has persistently attracted attention in the past two centuries. Its ill-posed nature (Istratov \& Vyvenko, 1999) means that there exist an infinite number of probability distributions of exponential components that could result in the same measured signal decay. To select a single solution from all the possible ones it is necessary to introduce appropriate prior information (i.e., regularisation) into the inversion, such as positivity, smoothness, or a predetermined form of the solution. One example is restricting the number of compartments but this does not always give an unambiguous answer (e.g. the discussion about fitting two compartments in Section 2).

A challenge specific to dMRI in the brain is that while it has marked sensitivity to differences in axon morphology (see Section 1), it has lower sensitivity to myelin (Beaulieu \& Allen, 1994) (Beaulieu, 2002). For example, the fractional anisotropy in the corpus callosum of the Shiverer mouse (with no myelination) is only $10 \%$ lower than in wild type mice (Nair et al., 2005). Diffusing species within the myelin itself will be largely invisible at the TEs typical of a diffusion-weighted experiment, since the macromolecules have ultrashort T2 (Fischer, Rinck, Van Haverbeke, \& Muller, 1990; Fischer, Van Haverbeke, Schmitz-Feuerhake, \& Muller, 1989), while water trapped within myelin has T2s lower than $40 \mathrm{~ms}$ (MacKay et al., 1994). Overall, the complexity of the white matter is such that one MRI contrast is unlikely to be sufficient to provide a complete characterisation of its microstructure. One might argue that understanding the precise origin of the signal change is not important, as long as we have access to tools that are sensitive to such a change. However, disentangling the contributions from myelin, axons and glial cells is essential to understand the pathophysiology of neurological disorders and therefore to develop treatments. In addition to disease (Bodini \& Ciccarelli, 2014; Lim \& Helpern, 2002; White \& Lim, 2010) (Lim \& Helpern, 2002), measuring white matter changes is also relevant for understanding the mechanisms underpinning plastic changes 
occurring to the brain as a consequence of maturation (Hüppi, 2010; Minati, Grisoli, \& Bruzzone, 2007; Sullivan \& Pfefferbaum, 2010) and training (Johansen-Berg, 2017) and lifestyle (Marks, Katz, Styner, \& Smith, 2011; Ritchie et al., 2017).

The lack of sensitivity to myelin of dMRI can be compensated by combining it with other techniques, such as multi-component relaxometry (MacKay et al., 1994), magnetization transfer (Henkelman, Stanisz, \& Graham, 2001; Sled et al., 2004; Wolff \& Balaban, 1989) and susceptibility imaging (Duyn, 2013; 2017; Duyn \& Schenck, 2017; Shmueli et al., 2009). While, in principle, the modalities of interest can be acquired independently and combined at the processing stage, the idea of manipulating more than one source of contrast within the same experiments is attractive. This type of "hybrid" experiment could be more time efficient, and minimise any source of bias, such as geometrical differences between acquisitions, and misregistration between voxels in the image (which might compromise the identification of sub-voxel compartments). This approach is also conceptually more straightforward from a processing perspective, because it enables the fitting of a single equation to the data.

The ideal hybrid experiment aims at reconstructing an $\mathrm{N}$-dimensional spectrum, where the $\mathrm{N}$ dimensions represent any combination of MR parameters of interest such as (T1, T2), ( $T 2$, Diffusion), ( $T 1$, Diffusion) in the case of $\mathrm{N}=2$ (Callaghan, Godefroy, \& Ryland, 2003), and potentially other combinations (e.g. diffusion and quantitative susceptibility mapping (Kaden et al. 2018). The complementarity of these MRI modalities implies that the separation of distinct environments can be improved by exploring their multidimensional correlation, compared with any of the 1D spectra (Celik, Bouhrara, Reiter, Fishbein, \& Spencer, 2013). While several multimodal experiments can be conceived to characterize the brain microstructure, here we will focus on those combining diffusion and relaxometry. This is partly justified by the abundance of examples in the literature, but also by the fact that this type of experiment is likely to benefit substantially from the use of ultra-high gradients. The popularity of the diffusion-relaxometry framework can be explained by the complementary nature of the information available with these techniques.

\section{BACKGROUND}

The first examples of "hybrid experiments" in biological tissue date back to the late 90 s, when attempts were made to establish the relationship between T2-species and diffusion behaviour (Peled, Cory, Raymond, Kirschner, \& Jolesz, 1999; Stanisz et al., 1997). The interest was prompted by the observation of multi-exponential behaviour for T2 and the TE dependency of some diffusion parameters, suggesting the presence of several compartments. Those experiments were mostly performed ex vivo, using some variant of the diffusion-weighted (DW) Carr-Purcell-Meiboom-Gill (CPMG) sequence, to assess whether compartments with shorter T2 (potentially associated with myelin) had diffusion properties consistent with this hypothesis. This acquisition consists of a standard pulsed-gradient spin-echo (PGSE) preparation (van 
Dusschoten, Moonen, de Jager, \& Van As, 1996) followed by a CPMG train of $180^{\circ}$ pulses. The T2-decay can thus be measured under variable diffusion-weighting conditions, enabling the apparent diffusion coefficient (ADC) of the water compartments corresponding to each $\mathrm{T} 2$ peak to be studied. Alternatively, the TE dependence could point to exchange with myelin water, which would make the TE dependence a potential marker for the degree of myelination (Lin et al., ISMRM 2017).

T1 spectra in single fibre populations have been reported as multi-exponential less consistently than T2, which has often thought to be caused by inter-compartmental mixing of water during the typically long measurement times. Nevertheless, multi-exponential T1 decay has been described e.g. (Does, Beaulieu, Allen, \& Snyder, 1998). Hybrid correlation experiments have shown to increase the potential of disentangling unique T1 values per compartment (e.g., T1-T2 relaxometry) (Deoni, Rutt, Arun, Pierpaoli, \& Jones, 2008). T1 compartmentalisation was also studied in combination with diffusion by adding inversion recovery (IR) to the DW-CPMG experiment (Andrews, Osborne, \& Does, 2006). Recent work by Benjamini et al. (Benjamini \& Basser, 2017) enabled the identification of 7 separate peaks by looking at the T2-Diffusion and T1-Diffusion space from ferret's spinal cord, each associated with a separate type of tissue ranging from glial to axonal, neuronal and myelin water. The difficulty of implementation (hampered by the long scan times and the SAR constraints) limited the pursuit of this type of experiment until recently, when the availability of better hardware and faster sequences, as well as of methods for optimising data sampling (Benjamini \& Basser, 2016) have made hybrid experiments feasible in vivo. De Santis et al., (De Santis, Assaf, Jeurissen, Jones, \& Roebroeck, 2016a; De Santis, Barazany, Jones, \& Assaf, 2016b) combine inversion recovery (IR) with dMRI for the purpose of mapping T1 (exploiting the IR preparation) along specific white matter tracts crossing within a single voxel (exploiting dMRI), assuming a mono-exponential T1 behaviour per fibre population. The feasibility of this approach in vivo was demonstrated by varying the inversion time (TI) for a moderate b-value of $1000 \mathrm{~s} / \mathrm{mm}^{2}$

\section{THE PROMISE}

One major limiting factor in current in vivo acquisitions is the low SNR. The SNR fundamentally limits the resolution of distinguishing exponentials with different decay rates (Bertero, Brianzi, \& Pike, 1999), and the resolution limit can therefore be expected to be worse for in vivo data having typically lower SNR than NMR data. Fortunately, there are several numerical procedures available that have shown to be able to reach the fundamental limit of distinguishing different exponentials (Istratov \& Vyvenko, 1999).

The lower SNR of in vivo data further challenges the exponential analysis as the stability of numerical methods and their sensitivity to noise becomes an increasingly important issue. While averaging of signals improves the SNR, this is obviously at the cost of an increased acquisition time. Regularisation is commonly employed in NMR correlation experiments to make the inversion less ill-conditioned, with Tikhonov 
regularization being a popular method (Venkataramanan, Yi-Qiao Song, \& Hurlimann, 2002)). Recent efforts to further optimise and regularise the estimation procedure include the use of compressed sensing (Bai, Cloninger, Czaja, \& Basser, 2015) (Cloninger, Czaja, Bai, \& Basser, 2014), marginal distributions (Benjamini $\&$ Basser, 2016) or spatial smoothness and constrained dictionary-based optimisation (Kim et al., 2017).

For in vivo human brain experiments, choosing a predefined form by explicitly modeling the contributions of different compartments is commonly performed to regularise the fit in diffusion microstructural imaging, and an extension of this to hybrid experiments is promising for the disentanglements of these compartments in terms of their diffusion and transverse relaxation properties (Veraart et al., 2017). However, recent findings show a correlation between parameters when the 'standard' model for diffusion without constraints (Section 2) was extended and applied in D-T2 hybrid experiments with limited b-values (up to $4000 \mathrm{~s} / \mathrm{mm}^{2}$ ) (Veraart et al., 2017). This raises concerns about the number of independent parameters that can be extracted from the available data (Reisert, Kellner, Dhital, Hennig, \& Kiselev, 2017; Lampinen et al. 2017). When performing such D-T2 experiments on clinical systems, higher b-values can only be achieved at the expense of long TEs, thereby significantly limiting the shortest TE and therefore SNR that can be achieved.

Depending on the specific MR contrasts one aims to exploit, ultra-high magnetic field gradients have the ability to provide a better SNR. In T2-D experiments, it is possible to maintain a relatively short TE for a given diffusion sensitisation, improving the SNR. In IR-PGSE sequences for T1-D correlations a similar reasoning applies. At the same time, ultra-strong gradients provide the unique opportunity to sample a much larger part of the multidimensional experimental parameter space than has previously been possible in vivo. For T2-diffusion experiments, ultra-high magnetic field gradients can be used to sample higher bvalues at shorter TEs (Figures 1 and 5). (Tax et al., 2017; Fan et al., 2017). Higher b-values could resolve the correlations between parameters (Veraart et al., 2017). Preliminary results show that high b-values are beneficial when trying to disentangle compartments (Tax et al., 2017) without fixing parameters to a priori values.

Reaching short TE is particularly important when attempting to capture myelin water contributions with very short T2 (MacKay et al., 1994) to the signal. Ultra-strong gradients combined with fast readout techniques, such as those mentioned in the Section 5 on high resolution, provide a promising outlook for characterising myelin water, thereby aiming at a more complete picture than has previously been possible with diffusion experiments. The wider TE range that can be sampled, however, requires a revisit of the signal modeling: if myelin is significantly contributing to the signal it should be included as an extra compartment in the model, and the role of exchange has to be re-examined. In addition, higher b-values 
with sufficient SNR in IR-PGSE experiments for D-T1, or even D-T1-T2 experiments enables a more detailed investigation of multi-component $\mathrm{T} 1$ relaxation per fibre population in vivo.

Future work will be directed towards carefully analysing the added information content in this previously inaccessible space, with the ultimate aim of developing joint tissue models. However, such an extensive sampling of the parameter space goes hand in hand with longer acquisition times, which makes it currently impractical for clinical translation. Compressive sensing (Bilgic et al., 2012; Doneva et al., 2010; Lustig, Donoho, Santos, \& Pauly, 2008; Lustig, Donoho, \& Pauly, 2007; Ning et al., 2015), “MR Fingerprinting” (Ma et al., 2013; Ma, Wright, Seiberlich, Gulani, \& Griswold, 2014) and other techniques have the potential to capture the essential information while further reducing acquisition times.

FIGURE 5 NEAR HERE

FIGURE 5: b-value vs echo time (TE) experiment for maximum gradient amplitudes of $300 \mathrm{mT} / \mathrm{m}$, allowing for high b-values (up to $7000 \mathrm{~s} / \mathrm{mm}^{\wedge} 2$ ) and low TE (down to $47 \mathrm{~ms}$ ). As a comparison, for the maximum b-value of 7000 $\mathrm{s} / \mathrm{mm}^{\wedge 2}$ used here, the minimum TE would be $75 \mathrm{~ms}$ on a $80 \mathrm{mT} / \mathrm{m}$ system and $105 \mathrm{~ms}$ on a $40 \mathrm{mT} / \mathrm{m}$ system, as can be derived from Figure 1. (a) shows the raw signal and (b) the logarithm of the signal, to illustrate that there is still signal at the highest $b$-value and TE.

\section{HIGH RESOLUTION DIFFUSION IMAGING AND TRACTOGRAPHY}

\section{THE CHALLENGE AND ITS IMPORTANCE}

Until now, diffusion imaging methods, applied to the study of the living human brain, have been significantly limited by their own low spatial resolution. However, there are several important applications that could benefit from high resolution acquisitions. E.g. it has been shown that important cortical changes at the macro-scale level can occur in numerous psychiatric, neurodevelopmental and neurodegenerative disorders, (Goldman et al. 2009; Zielinski et al 2014, D. S. Andrews et al., 2017; D’Anna et al. 2016; Ecker et al., 2016), but it has never been possible to show the corresponding micro-structural attributes, and especially in which specific laminae any changes occur. Enabling ultra-high resolution dMRI in this manner could yield new insights into the pathophysiology of a wide range of disorders. At the same time, better 
spatial resolution not only can benefit the quantification of microstructure diffusion properties, but could also significantly improve the accuracy of tractography reconstructions. By increasing the spatial resolution, different fibre pathways can be better disentangled and new minor white matter connections will emerge besides the major white matter fasciculi (Heidemann, Anwander, Feiweier, Knösche, \& Turner, 2012). Intralobar connections and u-shaped fibres (Catani et al., 2012; Catani et al. 2017) are essential short range connections that play an important role in distributing information across whole brain networks, but are currently significantly underestimated by most tractography and connectomics approaches.

\section{BACKGROUND}

Two of the main reasons for the low resolution of diffusion data are the intrinsic low SNR of MR diffusion data and the prevalence of single-shot acquisitions, selected due to the need to constrain total acquisition time, and to minimise motion artefacts (Turner, 1998; Turner et al., 1990). Although new post processing methods like super-resolution (e.g., Van Steenkiste et al., 2016), data interpolation (e.g.,Dyrby et al., 2014) and image quality transfer techniques (see Section 10) are today emerging as alternative ways to mitigate the lack of spatial resolution, improving data quality during acquisition remains of critical importance. Recent attempts have tried to overcome these limitations by combining the benefits of higher spatial resolution and SNR of 7T data with stronger diffusion weightings from 3T data (Sotiropoulos et al., 2016; Sotiropoulos et al., 2017). The use of ultra-strong gradients could potentially allow the same highresolution high-bvalue data to be collected, but using only one scanner.

\section{THE PROMISE}

Indeed, with the use of powerful gradient systems it is now possible to achieve a significant reduction of TE per unit b-value (Setsompop et al., 2013) and, if combined with field cameras (Wilm et al., 2017; 2015) and more echo-time efficient acquisition strategies (e.g. spiral readout), SNR can be further maximised and geometrical distortion significantly reduced. Compared to the use of weaker gradients at higher field (e.g., $70 \mathrm{mT} / \mathrm{m}$ at 7 Tesla), stronger gradients at lower field (e.g., $300 \mathrm{mT} / \mathrm{m}$ at 3 Tesla) allow the advantages of the longer T2, less $\mathrm{T} 2 *$ blurring and lower susceptibility distortion to be exploited. This in turn allows for higher spatial resolution and more anatomically accurate imaging and tractography reconstructions. Fan et al. (2017) recently proposed an interesting approach, dubbed 'HIBRID' (HIgh b-value and high Resolution Integrated Diffusion), that combines high resolution and high b-value data (collected with Connectom gradients), that allowed characterization of diffusion in the cortex without compromising white matter fiber information.

The adoption of new head RF-coils equipped with a high number of transmit and receiver channels can enable the use of high acceleration factors or the combination of multiple, advanced imaging techniques like multi-band (e.g., Larkman et al., 2001), parallel imaging methods (e.g. Larkman and Nunes, 2007), 
segmented-EPI (e.g. Frost et al., 2014); super-resolution (Van Steenkiste et al., 2016) and zoomed imaging e.g. Heidemann et al. 2012). One example of this new generation of imaging strategies is the Generalised Slice Dither Enhanced Resolution with Simultaneous Multislice ('gSlider-SMS') acquisition method (Setsompop, Ning \& Rathi, 2016; Setsompop et al., 2017). This technique combines multi-band acceleration with slice-dithering acceleration, as well as parallel and zoomed imaging, which has allowed the acquisition of full brain in vivo diffusion datasets at isotropic resolution of 750um within 50 minutes with a $b=1500$ $\mathrm{s} / \mathrm{mm}^{2}$ and 128 directions. At this resolution, cortical regions that are normally heavily affected by partial voluming with CSF or subcortical white matter are better resolved and the whole human cortex can be characterised with a thickness of several voxels. This can allow practical and more meaningful quantification of the cortical diffusion properties compared to previous studies where diffusion measures were inevitably limited by cortical regions of only one or two voxels width. If in the near future this technique will be also combined with microstructure imaging this will potentially enable the analysis in the living human brain of individual cortical layers with a more direct interpretation of neurological and psychiatric disorders.

An example is shown in Figure 6, with isotropic resolution data of 780 um, showing both the direction encoded fibre orientation (Pajevic and Pierpaoli, 1999) and the 'anisotropic power' obtained by taking the sum of the angular power spectrum of each spherical harmonic of even order I $\geq 2$ (Dell'Acqua et al. 2014)

\section{FIGURE 6 NEAR HERE}

Figure 6: Data obtained with the g-SLIDER technique (Setsompop et al., 2016; Setsompop et al., 2017 ) at 780 um. The left panel shows the direction-encoded colour map using standard convention (Pavevic and Pierpaoli 1999), with the lower row showing zoomed-in versions of the areas highlighted in yellow. The right hand panel shows the anisotropic power, processed according to Dell'Acqua et al. (Proc ISMRM 2014; Anisotropic Power Maps: A diffusion contrast to reveal low anisotropy tissues from HARDI data. p.6396), obtained from the sum of the angular power spectrum of each spherical harmonic of even order I $\geq 2$, resulting from spherical deconvolution of the HARDI signal

Furthermore, hybrid acquisitions can also help the integration of high resolution tractography data with microstructure modeling, potentially improving both tractography reconstructions by reducing confounding factor and allowing more refined "tractometry" (Bells et al. 2011) measurements based on-tract specific (Dell'Acqua, Simmons, Williams, \& Catani, 2013b; Raffelt et al., 2012) and other microstructure indices.

Together with high-resolution acquisitions, low anatomical distortions can also make anatomical regions normally precluded by susceptibility distortion (e.g. orbital-frontal and temporal regions) accessible, and therefore offer more accurate tractography reconstructions.

We caution that strong gradients per se are not a panacea for all the problems of tractography. While tractography today is a very powerful tool to segment the major white matter pathways there are still important limitations that prevent a reliable characterisation of their cortical connectivity. For example, Reveley et al. (2015) have pointed out with high resolution dMRI as much as "50\% of the cortical surface 
was effectively inaccessible for long-range diffusion tracking because of dense white matter zones just beneath the infragranular layers of the cortex". Stronger gradients neither do they directly solve the issue raised by Maier-Hein et al (2017), that "the geometry of many junctions in the simulated data set is too complex to be resolved by current tractography algorithms, even when given a perfect ground truth field of orientations".

Nevertheless, stronger gradients can indeed simplify the tractography problem. By pushing the spatial resolution of in vivo diffusion imaging below 1-mm voxel resolution and boosting SNR per unit time, this will doubtless lead to improvements in the fidelity of tractography reconstructions. Finally, high resolution in-vivo diffusion Imaging performed with a ultra gradient system has the potential to generate data that can be directly compared with equivalent high b-value experiments performed, until now, only on animal models or ex-vivo tissues in preclinical systems. E.g. the characterisation of the laminar organization of the cortex or deep gray matter done on ex-vivo samples (Dell'Acqua et al. 2013b, Leuze et al. 2014, Bastiani et al. 2016) could be translated to map the cortical, subcortical and cerebellar organisation of the living human brain and provide a bridge to help the interpretation of clinical and research results obtained on conventional clinical scanner at much lower resolutions.

\section{POST-MORTEM DIFFUSION IMAGING}

\section{THE CHALLENGE AND ITS IMPORTANCE}

Post mortem MRI, and in particular diffusion imaging of whole brain specimens, has attracted interest in recent years for several reasons. Diffusion MRI has tremendous potential to contribute to our knowledge of neuroanatomy, including macroscopic connectivity, local fibre architecture, and patterns of microstructural features across the brain. However, in vivo anatomical investigations with dMRI are limited by achievable spatial resolution and diffusion contrast, with improvements in both domains incurring reduced SNR. Postmortem MRI has potential to overcome this SNR challenge by enabling very long scan times (e.g. overnight or even several days). Indeed, dMRI with spatial resolution as high as $0.5 \times 0.5 \times 0.5 \mathrm{~mm}^{3}$ has been demonstrated in whole, post mortem human brains (Foxley et al. 2016). A second motivation for scanning post mortem brains is the ability to directly compare dMRI data to microscopy in the same tissue. Diffusion modeling generally assumes a relationship between underlying fibre architecture and diffusion signal, but all too often these assumptions are not validated (Dell'Acqua, Bodi, Slater, Catani, \& Modo, 2013a). Microscopy can be used to map these relationships explicitly, including the contribution of specific tissue compartments (Budde and Frank 2012; Schilling et al., 2016; Mollink et al., 2017). Post-mortem dMRI has been used in neurosurgical applications, both to validate tractography against histological stains (Mollink et al., 2016) and to support contraindications for surgical procedures (McNab et al., 2009). A more unusual, 
but no less important, motivation for post mortem imaging is the ability to study unusual specimens. Neuroanatomical studies are generally limited to a very narrow range of species (certain rodents and a few primates). Nevertheless, post mortem brain collections host a plethora of species that could not conceivably be studied in a laboratory environment, many of which are too rare to dissect. Post mortem MRI enables non-invasive study of these specimens not only in terms of gross anatomy, but also in terms of connectivity, which is increasingly of interest in comparative anatomy (Mars et al., 2014). For example, a recent study demonstrated the ability to reconstruct major white matter pathways from 100 -year-old brains of an extinct species, the Tasmanian tiger (Berns \& Ashwell, 2017), and another presented novel evidence for the pathways underpinning echolocation in dolphins (Berns et al., 2015).

In order to realize these goals, however, a number of challenges need to be overcome. As with other sections of this article, the primary challenge in realizing high spatial resolution is achieving high SNR. Although one can perform much longer scans than are possible in vivo, this in itself is insufficient to achieve major gains in spatial resolution. As with all MRI, improvements in spatial resolution come at an SNR cost that scales with the cube of voxel dimensions, while SNR scales with the square root of scan time. For example, if we aim to achieve $0.5 \times 0.5 \times 0.5 \mathrm{~mm}^{3}$ resolution without loss in SNR relative to a protocol achieving $1 \times 1 \times 1 \mathrm{~mm}^{3}$ spatial resolution in 1 hour, we would have to scan for 64 hours (nearly 3 days).

There are additional challenges presented by post mortem tissue. MR-relevant properties change in unfavourable ways in post-mortem tissue due to death and fixation, with $\mathrm{T} 1, \mathrm{~T} 2$ and diffusion coefficients all being significantly reduced (Shepherd, Thelwall, Stanisz, \& Blackband, 2009). T2 is reduced to 50-76\% of in vivo values (Pfefferbaum et al, 2004; Miller et al., 2011; Foxley et al., 2014), with the loss of T2 generally increasing with field strength from 1.5 to $7 \mathrm{~T}$. Reduced $\mathrm{T} 2$ is particularly problematic for conventional diffusion-weighted spin-echo (DW-SE) measurements, which require long TEs in order to achieve substantial contrast (b-value) (Miller et al., 2011). In addition, the diffusion coefficient is reduced to $20-50 \%$ of in vivo values in perfusion-fixed animal tissue (Sun et al, 2005; D'Arceuil et al., 2007), although measurements down to $10 \%$ of in vivo values have been reported in human brains with long post-mortem intervals (Miller et al., 2011). These reductions in ADC dictate that a higher b-value is needed to obtain contrast equivalent to current in vivo protocols, requiring even longer TEs and reducing signal even more. T1 values are less impacted (40-60\% of in vivo values) and in fact can improve image quality by enabling reduced repetition times. In general, these effects are heavily dependent on tissue preparation (Shepherd et al, 2009) and compounded by the long post-mortem interval between death and fixation in human brain specimens (Shepherd et al, 2009; D'Arceuil 2007b). These effects result in a net loss of SNR in post-mortem compared to in-vivo tissue (Fig, 7, left). 


\section{BACKGROUND}

There are a number of different approaches that have been taken to address these challenges. Small brains and tissue samples can be accommodated in small-bore scanners that are designed for imaging rodents (D'Arceuil, Westmoreland, \& de Crespigny, 2007; Dyrby et al., 2011). These systems benefit from stronger gradients than conventional clinical scanners, enabling short TEs to combat reduced T2. Moreover, small tissue samples can be soaked in buffer solution to wash out fixative, which is very effective in raising T2 values (which is predominantly caused by the short T2 of fixative itself) (Shepherd et al., 2009). Additional benefit can be made by doping the buffer to also alter T1 values (D'Arceuil et al., 2007). Unfortunately, fluid takes weeks to months to fully permeate human brains, and incomplete penetration of buffer leads to problematic hydration boundaries that obscure the intended contrast (Miller et al., 2011). In general, these approaches are only feasible for whole brains up to the size of a macaque (D'Arceuil et al., 2007) or pig (Dyrby et al., 2011). Early proof-of-concept for diffusion imaging of whole post-mortem human brains highlighted the potential to provide a direct validation against histopathology in disease, but generally suffered from modest image quality (Larsson et al 2004; Englund et al 2004; Pfefferbaum et al 2004).

It has been demonstrated that diffusion imaging of whole human brains can be achieved with minor modifications to a DW-SE sequence (in particular, using a 3D segmented readout) (Miller et al., 2011). However, it has also been demonstrated that significant gains in SNR and contrast can be obtained by moving away from conventional DW-SE sequences. One approach that has been explored in depth is a variant of steady-state free precession (SSFP), which has been demonstrated to provide substantial improvements in quality of tractography (Miller, McNab, Jbabdi, \& Douaud, 2012). In theory, DW-SSFP achieves greater efficiency than any other technique by re-using the signal created in one excitation over several acquisition blocks, decoupling contrast from TE. Unfortunately, DW-SSFP is also extremely gradient intensive, leading to heating constraints. Moreover, the signal has a complicated dependence on T1, T2, diffusion coefficient and acquisition parameters, and does not have a well-defined diffusion time (McNab \& Miller, 2010). Another alternative that has recently been revisited for post mortem imaging is diffusionweighted stimulated echoes with EPI readout (DW-STE) (Rane, Nair and Duong, 2010; Lundell, Alexander, \& Dyrby, 2014; Merboldt, Hänicke, \& Frahm, 1991). This sequence has many of the benefits of DW-SSFP, including decoupling of the b-value from the TE, while retaining a well-defined diffusion time and simple 
signal dependence. The main disadvantage of DW-STE is loss of $50 \%$ of the signal in the formation of the echo. Finally, ultra-high field strength (7 Tesla and above) has been demonstrated to provide further improvements for both DW-SSFP (Foxley et al., 2014) and DW-STE (Fritz 2017), although RF transmit inhomogeneity presents a further challenge at these field strengths.

\section{THE PROMISE}

As outlined above, the primary need for alternate acquisition approaches in post mortem brain imaging relates to the limitations of current clinical scanners, most specifically the relatively low maximum gradient strength. In pre-clinical (rodent) scanners, the high available gradient strength (typically $300-1000 \mathrm{mT} / \mathrm{m}$ ) enables the use of DW-SE with high SNR. This enables the acquisition of data that are maximally compatible with in vivo approaches, avoids the $50 \%$ signal loss of DW-STE and has a simple signal dependence with well-defined diffusion time. The availability of similar gradient strength in a scanner large enough to accommodate whole, human brains could enable anatomical investigations of unprecedented quality, providing high spatial resolution across the entire brain and in larger cohorts than can be studied with other methods. Figure 7 shows predicted improvements in SNR for post-mortem dMRI using spin-echo acquisition with $300 \mathrm{mT} / \mathrm{m}$ compared to $80 \mathrm{mT} / \mathrm{m}$.

\section{FIGURE 7 NEAR HERE}

Figure 7. Predicted improvements for post-mortem dMRI using spin-echo acquisition with $300 \mathrm{mT} / \mathrm{m}$ compared to $80 \mathrm{mT} / \mathrm{m}$. The horizontal axis plots signal contrast in terms of $b D$, matching in-vivo and post-mortem conditions for differences in diffusion coefficients (assuming $D=1 \mu \mathrm{m}^{2} / \mathrm{ms}$ and $0.3 \mu \mathrm{m}^{2} / \mathrm{ms}$ for in-vivo and post-mortem tissue, respectively). Left: SNR efficiency predictions at $3 T$ for post-mortem and in-vivo tissue for optimized protocols with different gradient maxima. The overall lower SNR efficiency for post-mortem tissue is driven by reduced $T 2$ and the need for longer TE to achieve equivalent contrast (assuming T2=80 and $45 \mathrm{~ms}$ for in-vivo and post-mortem tissue, respectively). Right: To match SNR efficiency in post-mortem tissue, one can take advantage of the ability to scan for longer periods than in living subjects. For $80 \mathrm{mT} / \mathrm{m}$ gradients, the fractional increase ranges from a factor of 511 over this range, while $300 \mathrm{mT} / \mathrm{m}$ gradients can achieve the same with more modest increases in scan time of only 2-3. Many post-mortem protocols scan for 12-24 hours, where a typical in-vivo protocol might scan for 30 minutes, thus far exceeding these fractional increases.

By overcoming the primary challenges associated with SNR, these scanners should also enable investigation of more subtle diffusion-based contrast in post-mortem brains, such as the microstructural features. This enables validation of these features against direct measurements based on microscopy (Mollink et al., 2017), comparison between different microstructural models with a gold standard for truth (Schilling et al., 2018), and critical assessment of the current shortcomings in dMRI modeling (Reveley et al. 2015). Finally, the very nature of such datasets with unusually high quality in multiple dimensions (spatial resolution, contrast and linkage to microscopy) has promise to provide novel findings. In recent years, post- 
mortem dMRI has enabled new insights into neurology (McNab et al., 2009; Kolasinski et al., 2013; Modo et al., 2016); demonstrated the ability to visualize and segment structures in human brain that are currently inaccessible in vivo (Augustinack et al., 2010; Aggarwal et al., 2013; Augustinack et al., 2014); provided the ability to detect gray matter microarchitecture features (Leuze et al. 2012; Kleinnijenhuis et al., 2012; Aggarwal et al., 2015; Kleinnijenhuis et al., 2015); and established a unique toolkit for comparative anatomy of a broad range of species (Jbabdi et al., 2013; Mars et al., 2014; Berns et al., 2015; Berns \& Ashwell, 2017). With improvements in both hardware and the approaches outlined above, post-mortem MRI could provide unique and rich insight into neuroanatomy.

\section{DIFFUSION-WEIGHTED MRS}

\section{THE CHALLENGE AND ITS IMPORTANCE}

While dMRI has predominantly focused on the diffusion of water molecules in tissue, the problem is that water is ubiquitous in tissue and exists in all compartments (e.g, intra-cellular and extra-cellular). It is this fundamental fact that makes it difficult to disentangle different tissue subcompartments (see Sections 1 and 2). Diffusion-weighted Magnetic Resonance Spectroscopy (DW-MRS) uniquely enables specific characterization of tissues such as the brain in vivo by quantifying the diffusion properties of MRobservable metabolites. Many brain metabolites are predominantly intracellular, and some of them are preferentially localized in specific brain cell populations (e.g., neurons and glia). Since many developmental processes, for instance plasticity and aging, or pathological processes, such as neurological diseases, are characterized by modulations of specific cellular types and their microstructures, and since water signals are not necessarily representative of any specific compartment, metabolite signals can serve as biomarkers with enhanced specificity (Palombo, Shemesh, Ronen, \& Valette, 2017b). However, DW-MRS measurements are extremely challenging, from the acquisition to the analysis and quantification stages. In vivo DW-MRS pulse sequences must achieve several goals simultaneously: (1) adequate diffusion weighting; (2) efficient and accurate signal localization to the volume of interest (i.e., MRS voxel, Figure 8a); and (3) robust acquisition of a free induction decay (FID) to obtain the chemical shift spectral dimension.

Here, we briefly discuss the importance of addressing point (1) by developing ultra-high gradient systems, and therefore the applications that would be made possible in humans in vivo. Since metabolite apparent diffusion coefficient, or ADC, is about 5-10 times smaller than water $\left(0.15-0.25 \mu \mathrm{m}^{2} / \mathrm{ms}\right.$ versus $0.7-1.5$ $\mu \mathrm{m}^{2} / \mathrm{ms}$ for water in tissue (Ellegood, Hanstock, \& Beaulieu, 2011)), larger b-values are required for metabolite signals in order to attenuate the signal ( $\exp [-b \mathrm{ADC}])$ to the same extent. For a conventional experiment, like the measurement of the ADCs of metabolites, which requires 'moderate' $b$ values, it is 
necessary to have a gradient strength at least 2-3 times stronger than that typically used for water (i.e. at b 1000 s/mm²). Moreover, recent preclinical studies (Assaf \& Cohen, 1999; Cohen \& Assaf, 2002; Ellegood, McKay, Hanstock, \& Beaulieu, 2007; Kroenke, Ackerman, \& Yablonskiy, 2004; Ligneul, Palombo, \& Valette, 2017; Palombo, Ligneul, Hernandez-Garzon, \& Valette, 2017a; Yablonskiy \& Sukstanskii, 2010) have shown that when the ultra-high b-value domain (i.e., $b>5,000 \mathrm{~s} / \mathrm{mm}^{2}$ ) or ultra-short diffusion time domain (i.e. < $5 \mathrm{~ms}$ ) are accessible, DW-MRS techniques offer huge potential for the non-invasive estimation of brain cellspecific microstructural properties (e.g., microscopic anisotropy, size and surface-to-volume ratio of neuronal and glial cell projections) that are out of reach of existing MRI techniques that are based solely on water diffusion.

\section{BACKGROUND}

In the early days of DW-MRS, due to the relatively low maximal gradient strength available on MRI scanners, the stimulated echo-based DW-STEAM pulse sequence (called for simplicity PGSTE) (Moonen, van Zijl, Le Bihan, \& DesPres, 1990; Posse, Cuenod, \& Le Bihan, 1993) (Figure 8-b) was the most convenient way to reach sufficiently high b-values in vivo (Ellegood et al., 2011) without excessive signal loss due to transverse relaxation, since during the mixing time, magnetization relaxes according to $T_{1}$ ( typically 1-1.5 s for metabolites at 3 T (Mlynárik, Gruber, \& Moser, 2001)). While this approach is advantageous for many reasons (Cao \& Wu, 2017; Nicolay, Braun, Graaf, Dijkhuizen, \& Kruiskamp, 2001; Palombo, Shemesh, Ronen, \& Valette, 2017b; Ronen \& Valette, 2007), the most significant drawback at the moderate magnetic field strength of clinical scanners (typically $3 \mathrm{~T}$ ) is the loss of half the magnetization (see also Section 6). Therefore, a spin echo sequence might be preferred, as sensitivity is one of the major issues of MRS in general, and of DW-MRS in particular. Nevertheless, in the spin echo sequence, diffusion time and TE are coupled. This, together with signal loss due to $T_{2}$ relaxation (typically 150-300 ms for metabolites at $3 \mathrm{~T}$ (Mlynárik et al., 2001)) occurring during the TE, imposes an upper limit on the longest diffusion time achievable. Hence, in order to translate advanced DW-MRS techniques to in vivo measurements in humans using clinical 3 T scanners, and to achieve good sensitivity and very high b-values, it is necessary to have access to a gradient strength that is 5-10 times stronger than that typically used for water.

The first spin echo DW-MRS sequences were based on PRESS (point-resolved spectroscopy), and were performed on preclinical systems with gradient strengths sufficient to reach sufficiently high b-values (Dijkhuizen, de Graaf, Tulleken, \& Nicolay, 1999; van der Toorn, Dijkhuizen, Tulleken, \& Nicolay, 1996). The sequence was subsequently modified for clinical implementation at $1.5 \mathrm{~T}$ by applying a bipolar gradient scheme (Kroenke et al., 2004), thus taking advantage of the twice-refocused spin echo sequence to increase maximal achievable diffusion-weighting. As with DT-MRI, it is also possible to assess the macroscopic anisotropy of metabolite diffusion in the brain tissue by using a minimum of six diffusion gradient directions. This technique is called diffusion tensor spectroscopy (DT-MRS, also known as DTS), 
and permits the estimation of the elements of the macroscopic diffusion tensor of typical MRS-visible metabolites, such as NAA ( $\mathrm{N}$-acetyl-aspartate), tCr (total Creatine) and tCho (total Choline). A DW-PRESS sequence with a specific gradient scheme has also been proposed to provide single-shot isotropic diffusionweighting (i.e., weighting by the trace of diffusion tensor) (de Graaf, Braun, \& Nicolay, 2001).

Several metabolite ADCs have been measured in the healthy human brain (Ellegood et al., 2011; Ellegood, Hanstock, \& Beaulieu, 2006; Posse et al., 1993) with ADC values for NAA, tCr and tCho being around 0.15$0.20 \mu \mathrm{m}^{2} / \mathrm{ms}$. DT-MRS has also been used to assess the anisotropy of metabolite diffusion in human brain (Ellegood et al., 2006; Upadhyay, Hallock, Ducros, Kim, \& Ronen, 2008). FA values of 0.48-0.72 were reported for NAA (Ellegood et al., 2006; Upadhyay et al., 2008), 0.56-0.73 for tCr (Ellegood et al., 2006) and 0.59-0.74 for tCho (Ellegood et al., 2006) in white matter regions such as the corpus callosum, corticospinal tract and arcuate fasciculus. A first examination of cortical gray matter (GM) yielded an unexpectedly high degree of anisotropy $(F A=0.53-0.79)$ for all three metabolites, similar to those seen in white matter (Ellegood et al., 2006). However, a later study by Ellegood et al. (Ellegood et al., 2011), showed that the high FA measured in GM was an artefact caused by the poor two-point slope estimation of the signal intensity versus b-value when using sub optimal b-values. Using a maximum b-value of $\sim 5 \mathrm{~ms} / \mu$ ? $\mathrm{m}^{2}$, instead of the much lower $1.8 \mathrm{~ms} / \mu$ ? $\mathrm{m}^{2}$ used in (Ellegood et al., 2006), lower FA values of $0.25,0.30$ and 0.28 for NAA, tCr and tCho, respectively, were indeed estimated in human occipital GM.

Numerous valuable studies have been recently using DW-MRS on clinical scanners (mostly at $3 \mathrm{~T}$ and $7 \mathrm{~T}$, and maximum gradient strength of $20-60 \mathrm{mT} / \mathrm{m}$ ) to measure metabolites ADC and DT, and to provide complementary information to various MRI metrics (DTI metrics, $T_{1}, T_{2}, M T R$ ) for an improved diagnosis and prognosis of different brain pathologies, (for a more comprehensive review, we refer the reader to (Cao \& Wu, 2017; Nicolay et al., 2001; Palombo, Shemesh, Ronen, \& Valette, 2017b; Ronen \& Valette, 2007)). So far, DW-MRS has been applied to study brain activity (Branzoli, Techawiboonwong, Kan, Webb, \& Ronen, 2013), cerebral ischemia (Harada et al., 2002; Zheng, Liu, Fang, Wang, \& Zhang, 2012), cerebral tumors (Zheng et al., 2012), normal aging (Zheng et al., 2012) (Branzoli et al., 2013), schizophrenia (Du et al., 2013) and multiple sclerosis (Wood et al., 2017; 2012) in human. For example, metabolite DT-MRS was used together with water-based DT-MRI to investigate axonal damage and to distinguish axonopathy from other processes such as inflammation, edema, demyelination and gliosis in multiple sclerosis (Du et al., 2013). Glial and axonal changes in neuropsychiatric systemic lupus erythematosus were assessed using metabolite DW-MRS, showing that intracellular alterations, and in particular changes in glia, as evidenced by an increase in the $\mathrm{ADC}$ of tCho and $\mathrm{tCr}$, correlate with systemic lupus erythematosus activity (Ercan et al., 2016).

\section{THE PROMISE}


The possibility of employing stronger gradient systems on human scanners (McNab et al., 2013; Setsompop et al. 2013), would help to improve DW-MRS measurements and enable the translation to humans of cutting-edge techniques that, at the moment, are limited to preclinical applications due to the weaker gradients available on clinical systems. It has been shown that metabolites ADC and DT metrics can already be estimated in vivo in human brain using clinical scanners with weaker gradients ( 40-60 mT/m). However, in order to have robust and reliable estimates, reduced artefacts and good sensitivity at moderate magnetic fields ( $3 \mathrm{~T}$ ), it may be extremely advantageous to have strong gradient systems to reach high $\mathrm{b}$ values (> $3000 \mathrm{~s} / \mathrm{mm}^{2}$ ), while keeping the TE and the gradient duration as short as possible (Ellegood et al., 2011; Wood et al., 2015). Moreover, stronger gradient systems enable shorter diffusion times, which in turn reduces the variability of the measurement due to fluctuations in the signal due to the heartbeat (i.e., blood flow peaks during the diffusion time), which can become significant for $b>3000 \mathrm{~s} / \mathrm{mm}^{2}$. While cardiac gating could, in principle, be used, it becomes ineffective for very long diffusion times (more than hundreds of $\mathrm{ms}$ ).

While ADC and DT derived metrics from non-water metabolites can be very useful in some clinical applications, they are only indirect reporters of the underpinning tissue microstructure (see Section 3 for discussion on the lack of specificity). Earlier strategies for more directly quantifying tissue microstructure from metabolite DW-MRS have involved q-space MRS (Assaf \& Cohen, 1998; Cohen \& Assaf, 2002), the quantification of the average propagator and some of its time-dependent properties. Recent work has described a first attempt to model metabolites diffusion at high $\mathrm{q} / \mathrm{b}$ values, taking into account the cellular structure, and to estimate the microscopic anisotropy and diameter of cellular projections (Kroenke et al., 2004; Palombo, Ligneul, \& Valette, 2016a; Yablonskiy \& Sukstanskii, 2010). These preliminary studies involved ultra-high diffusion weightings, obtained by employing ultra-strong diffusion gradient systems, and so to date have only been performed in vivo in preclinical applications. Nevertheless, these pioneering studies at ultra-high $\mathrm{q} / \mathrm{b}$ values have paved the way for some exciting possibilities enabled by implementing DW-MRS with ultra-strong diffusion gradients. Recent in vivo studies in mouse brain (Lignuel et al. 2016; Ligneul et al. 2017) and numerical simulations (Palombo, Ligneul, Hernandez-Garzon, \& Valette, 2017a) showed that measurements of metabolites' diffusion-weighted signal involving high gradient strength (i.e., PGSTE at $b>5,000 \mathrm{~s} / \mathrm{mm}^{2}$ and oscillating-gradients spin echo, OGSE, at high frequencies and high $b$ values: $f$ $>100 \mathrm{~Hz}$ and $b>1500 . \mathrm{s} / \mathrm{mm}^{2}$ ) help to address some of the limitations of water-based DW-MRI techniques (Figure 8c). These limitations include the lack of cell-specificity, and poor sensitivity to fine cellular microstructure, such as the density of small cell fibres (diameter $<3 \mu \mathrm{m}$ ) or neuronal spines. DW-MRS at high gradient strength would be a first necessary step towards the definition of new cell-specific biomarkers of the alteration of tissue microstructure due to neurodegeneration. Preliminary in vivo work using the APP/PS1/tauP301L mouse model of Alzheimer's disease (Ligneul et al. 2016), and the ciliary neurotrophic factor induced mouse model (Ligneul et al. 2017) showed that it is possible to use DW-MRS at 
ultra-high b-values to measure changes in the size of neuropils due to the induced neurodegenerative process in a cell-specific fashion, distinguishing between glial and neuronal cell alterations. Combining high $b$ values PGSTE and high frequency OGSE measurements, it would also be possible to measure changes in cellular projections' surface-to-volume ratio and dendritic spine density (Palombo, Ligneul, HernandezGarzon, \& Valette, 2017a), which may be extremely useful in the diagnosis and prognosis of pathologies such as autism (Hutsler \& Zhang, 2010) and essential tremor (Louis et al., 2014). As shown in Figure 8c, the Connectom scanner's ultra-strong gradient hardware would help filling the gap between preclinical and clinical scanners, opening a unique possibility to start investigating, in vivo in humans, a region at high $b$ values and high frequencies (green areas in Figure 8

c), currently inaccessible using clinical scanner and similar PGSTE or OGSE protocols.

\section{FIGURE 8 NEAR HERE}

\section{Figure 8}

Example of advanced DW-MRS experiments currently achievable on preclinical scanners only, due to a necessary maximum gradient strength, $G_{\max }>100 \mathrm{mT} / \mathrm{m}$. (a) Typical size of the MRS voxel $\left(6 \times 2.5 \times 5 \mathrm{~mm}^{3}\right)$ required to reach adequate signal-to-noise ratio of the diffusion-weighted signal of the relevant brain metabolites, like NAA and Myoinositol. (b) Advanced DW-MRS experiments require optimized PGSTE and OGSE sequences and ultra-strong gradient hardware in order to reach high $b$ values $\left(>5,000 \mathrm{~s} / \mathrm{mm}^{2}\right)$ and high frequencies $(w>100 \mathrm{~Hz})$. Adapted from Reynaud (2017). (c) Examples of DW-MRS signal decays as a function of $b$ and ADC as a function of $w^{-1 / 2}$ for two different metabolites: NAA (mostly compartmentalized in neurons) and Myo-inositol (mostly compartmentalized in glia), obtained by using PGSTE and OGSE sequences with $G_{\max } \sim 800 \mathrm{mT} / \mathrm{m}$ and respectively $T E / b_{\max }=32 \mathrm{~ms} / 60,000$ $\mathrm{s} / \mathrm{mm}^{2}$ and TE $/ b_{\max }=60 \mathrm{~ms} / 1,200 \mathrm{~s} / \mathrm{mm}^{2}$ (adapted from [Palombo et al. (2017a)). Coloured areas represent the regions accessible by current clinical scanners with $G_{\max }=80 \mathrm{mT} / \mathrm{m}$ (red areas); the Connectom scanner with $G_{\max }=300 \mathrm{mT} / \mathrm{m}$ (green areas) and a preclinical scanner with $G_{\max }=800 \mathrm{mT} / \mathrm{m}$ (blue areas), using the same $T E / b_{\max }$ values reported in (b).

Although much of the development of DW-MRS has been done on preclinical systems, it is clear that the technique may become a very powerful and useful clinical diagnostic tool. Systems operating at moderate static magnetic field (e.g. 3 T), equipped with strong gradient units $(>100 \mathrm{mT} / \mathrm{m})$ are ideal for investigating short-range features of brain tissue microstructure using DW-MRS at intermediate diffusion times (<200 $\mathrm{ms}$ ) and high $\mathrm{b}$ values $\left(>5,000 \mathrm{~s} / \mathrm{mm}^{2}\right)$, or ultra-short diffusion times $(<5 \mathrm{~ms})$ and moderate $\mathrm{b}$ values (b>1500 s/mm ${ }^{2}$ ) (Figure 8-c). As previously discussed, this would provide enough sensitivity to quantify changes in cellular features, like the diameter and surface-to-volume ratio of cellular projections, and improve the early diagnosis of neurodegenerative disease (Valette et al. 2018). On the other hand, an important constraint arising from the low concentration of metabolites, compared to water $\left(\sim 10^{4}\right.$ times 
less), is that the spatial resolution i.e., the voxel sizes, and acquisition time are generally much larger than those of DW-MRI (Figure 8-a), representing a major hurdle to the clinical applicability of DW-MRS. Higher static magnetic fields (e.g., $7 \mathrm{~T}$ ) provide improved spatial resolution and reduced acquisition time, facilitating the characterisation of long-range features of brain tissue microstructure using DW-MRS at long diffusion times (>200 ms) and moderate b values $\left(<5000 \mathrm{~s} / \mathrm{mm}^{2}\right)$ (Najac, Branzoli, Ronen, \& Valette, 2016; Palombo et al., 2016b). However, the weaker gradients available on these systems ( $<100 \mathrm{mT} / \mathrm{m})$, and the challenges of achieving a well-shimmed homogenous field, remain an obstacle to performing high b-value investigations to measure changes in the cellular short-range morphology.

Hence, there is still much to be done in terms of acquisition methodology. The critical steps towards the full realisation of the potential of DW-MRS in the clinic are the development of: 1 ) better and stronger gradient systems; 2 ) more sensitive RF coils; 3 ) higher static magnetic fields.

\section{CANCER IMAGING / TUMOUR CHARACTERIZATION}

\section{THE CHALLENGE AND ITS IMPORTANCE}

The dMRI signal is potentially sensitive to a number of features of the tumour microenvironment that are relevant to diagnosis and assessment of treatment response. The promise of specific measurements of such properties is that they provide direct insight into key aspects of pathology that are only indirectly and, therefore potentially ambiguously, reflected using heuristic parameterisations such as ADC or kurtosis (e.g., Bourne, 2015; K. M. O'Connor, Barest, Moritani, Sakai, \& Mian, 2013). Getting access to such information over the whole organ of interest, (rather than measurements available from a needle biopsy, for example), would pay huge dividends for diagnosis, longitudinal monitoring and evaluation of treatment response.

\section{BACKGROUND}

As illustrated in Figure 9, different models and acquisitions have been used to estimate or evaluate the sensitivity to: cell size and intracellular volume fraction (Panagiotaki et al., 2014, 2015; Reynaud et al., 2016; Jiang et al., 2017), nuclear-to-cell volume ratio (Xu et al., 2009), water exchange (Lampinen et al., 2017b), and vascular volume fraction (Panagiotaki et al, 2014, 2015). Intra- and extra-cellular diffusion coefficients have been either estimated (Reynaud et al., 2016; Jiang et al., 2017) or fixed (Panagiotaki et al, 
$2014,2015)$, and the pseudo-diffusion coefficient associated with blood flow has been estimated (Panagiotaki et al, 2014).

\section{FIGURE 9 NEAR HERE}

Figure 9. Literature examples of microstructural imaging in cancer. (a) VERDICT yields estimates of compartment volume fractions, cell size, and the pseudo-diffusion coefficient in preclinical colorectal xenografts, demonstrating sensitivity to treatment-induced changes (Panagiotaki et al., 2014). (b) IMPULSED combines PGSE and OGSE measurements to estimate cell size, and compartment volume fractions and diffusivities in preclinical colorectal xenografts, with dMRI-derived cellularities correlating with histology (Jiang et al., 2017). (c) POMACE models the time-dependent diffusivity from PGSE and OGSE data to estimate microstructural properties in preclinical gliomas, with cell sizes consistent with independent measurements (Reynaud et al., 2017.). (d) FEXI shows differences in the apparent exchange rate between meningiomas and astrocytomas (Lampinen et al., 2017b).

Such additional pathological specificity then has the potential to provide robust biomarkers $\left(\mathrm{O}^{\prime}\right.$ Connor et al., 2017) for a range of potential applications, including:

- Distinguishing tumour/non-tumour tissue: Often tumours exhibit alterations in cell packing that can potentially be identified using dMRI. Vasculature is also typically abnormal in cancer.

- Distinguishing between tumours of different grades and characterizing tumour extent: As tumours progress, they exhibit phenomena such as infiltration of surrounding tissue, leading to a mixture of healthy and tumour cell types at the tumour margins, in addition to internal heterogeneity of cellularity and vascularity.

- Predicting treatment response: Microstructural characterisation of pre-treatment tumours may aid interpretation of, or offer improved sensitivity over, the observation of higher baseline ADC being linked to poorer treatment response (Tam et al., 2013), for example by providing a means of attributing differences in $A D C$ to differences in necrosis or cellularity

- Early assessment of response: Cell stress occurring early during treatment induces changes in the flux of water across cell membranes, leading to changes in cell size and packing density. As cell death ensues, further changes in these parameters may occur in advance of gross tumour size change.

- Distinguishing pseudo-progression from true progression: Apparent tumour growth (typically in the context of brain tumours) following treatment can be associated with changes in contrast enhancement caused by damage to the tumour tissue and potentially to surrounding non-tumourous tissue. This increase in apparent tumour volume would classically be associated with tumour progression but in reality is a sign of response to treatment. The associated cell death, however, is reflected in changes in cell packing, which should be discernible with dMRI.

\section{THE PROMISE}

The above measurements require the fitting of models of water compartmentalisation and exchange to data obtained at a range of diffusion times and gradient strengths. As noted several times already, current 
clinical MRI systems lack the gradient performance to enable broad coverage of the $G-\Delta$ space (see Figure 1b), leading to model parameter imprecision, poor model fitting and the use of overly simplistic models.

As is the case for all microstructure imaging using dMRI, access to higher gradient strengths enables higher $\mathrm{SNR} /$ shorter TE for a given $b$-value, as well as larger $b$-values for shorter diffusion times. Although differences in rise times for $80 \mathrm{mT} / \mathrm{m}$ and $300 \mathrm{mT} / \mathrm{m}$ gradients mean that a wider range of $\Delta$ values are in principle achievable with the former, the lower $\Delta$ values suffer from very low $b$-values (e.g., $187 \mathrm{~s} / \mathrm{mm}^{2}$ for $\partial=5 \mathrm{~ms}, \Delta=18 \mathrm{~ms}, \mathrm{G}=80 \mathrm{mT} / \mathrm{m}$, rise time $=0.4 \mathrm{~ms}$; see Figure $1 \mathrm{~b}) .300 \mathrm{mT} / \mathrm{m}$ gradients make shorter diffusion times ( $\Delta \sim 24 \mathrm{~ms}$ ) more practical, as large b-values are still achievable (e.g. $8693 \mathrm{~s} / \mathrm{mm}^{2}$ for $\partial=8 \mathrm{~ms}, \Delta=24 \mathrm{~ms}$, $\mathrm{G}=300 \mathrm{mT} / \mathrm{m}$, rise time=3.6 ms; see Figure 9). As the cell sizes in cancer are typically greater than the sizes of axonal features of interest in most neurological dMRI, with the significant exception of increased SNR, the advantages of higher $G$ are not necessarily as clear for modeling of basic cell dimensions. However, heuristic quantification of the dMRI signal in cancer can also benefit from better gradient performance. For example, recent investigation of diffusion time-dependent ADC in prostate tumours suggests shorter $\Delta$ values yield greater contrast between high- and low-grade tumours (Lemberskiy et al., 2017).

\section{Cellular and sub-cellular structure}

Combining stronger gradients with OGSE gradient waveforms increases sensitivity to differences in the ratio of nuclear volume to cell volume (Xu, Does, \& Gore, 2009), the relevance of which is that nuclear size is related to tumour grade, and can change with therapy. However, the rise time requirements imposed in order to meet safety constraints (see Section 11 below) limits the OGSE frequencies which can be practically achieved ( $\sim 0 \mathrm{~Hz}$ for a $3.6 \mathrm{~ms}$ rise time). This suggests that the main benefit of stronger gradients for OGSE will be the ability to achieve higher b-values for low frequencies, as opposed to providing access to higher frequencies. Alternative gradient waveforms, including those used for spherical tensor encoding (Eriksson et al., 2013; Lasič et al., 2014) can also provide deeper insight into tissue than DTMRI, characterizing differences in microscopic anisotropy (Szczepankiewicz et al., 2015; 2016). The relatively long duration of the waveforms required to play out a spherical tensor encoding mean that SNR is compromised on commonly-available hardware, but implementation with stronger gradients reduces the duration for which each 'lobe' needs to be played out. In turn, this conveys a reduction in TE and a concomitant boost in SNR (approximately $50 \%$ at $300 \mathrm{mT} / \mathrm{m}$ compared to $80 \mathrm{mT} / \mathrm{m}$ ).

\section{Beyond cellularity - vasculature and other microstructure}

Flow and vascular volume fraction have been estimated using model-based approaches for interrogating the dMRI signal in tumours. The simplest approach is to employ the intra-voxel incoherent motion (IVIM) model (lima \& Le Bihan, 2016; Koh, Collins, \& Orton, 2011; Le Bihan et al., 1986; Marzi, Piludu, \& Vidiri, 2013), although more sophisticated approaches such as VERDICT (Panagiotaki et al., 2014) offer the 
promise of greater specificity of signal characterisation in terms of the relative contribution of different cellular architecture and vasculature. An alternative pragmatic approach is to identify the contribution of the vasculature by combining flow- and non-flow-compensated acquisitions (Wetscherek et al., 2015; Ahlgren et al., 2016). Finally, beyond characterising the intracellular and vascular spaces, dMRI can provide information about extracellular matrix components such as collagen1 (Col1) fibres, which facilitate macromolecular transport within tissue and dissemination of cancer cells. It has already been shown that metrics derived from DT-MRI (FA / MD) are sensitive to differences in Col1 fibres (Kakkad et al., 2016).

\section{Measurement validation}

Measurement validation is obviously an essential step in the development of imaging biomarkers for cancer (O'Connor et al., 2017). Ultra-strong gradients are not currently available to the clinical cancer imaging community, so methods that work only with access to such systems are unlikely to generate useful biomarkers (although see Section 10 on Image Quality Transfer). As such, the benefits of increased gradient strengths for clinical cancer imaging are currently likely to be indirect, derived from using such high performance systems to provide validation of methods that can be implemented on lower-performance systems. Indeed, this may be the only way to demonstrate equivalence between biomarker information available on human MRI systems, and on preclinical scanners. With preclinical systems, although gradient strength is high, the field strength is typically also larger, and there are differences in coils, relaxation times, and resolution. The ultra-strong gradient system, coupled with a $3 T$ human system, allows us to match other (non-diffusion parameters) to clinical situations, which is useful particularly when histological validation is unavailable or inadequate. Finally, we recognize of course that ultra-strong gradient hardware is not yet widely available, so the immediate benefits for cancer care wil be indirect. However, in Section 10 , we speculate as to how measurements on such systems can inform more 'routine' clinical assessments on more standard hardware.

\section{FUNCTIONAL DIFFUSION IMAGING}

\section{THE CHALLENGE AND ITS IMPORTANCE}

Measuring neuronal activity non-invasively is fundamental to understanding the brain and its dysfunction occurring in disease. Direct measurements of the effects of neuronal current can be achieved with neurophysiological techniques, such as electro-encephalography (EEG) and magneto-encephalography (MEG). These methods, however, suffer from limited spatial resolution, and their sensitivity is typically restricted to cortical activity. Functional MRI (fMRI) based on blood-oxygenation level-dependent (BOLD) 
contrast on the other hand provides a very indirect estimation of neural activation through related haemodynamic and metabolic changes (neurovascular coupling) (Logothetis \& Wandell, 2004). One of the consequences is that the BOLD signal originates from both capillaries close to the site of neuronal activity, and larger vessels or distant draining veins (Lai, Glover, \& Haacke, 2000; Olman, Inati, \& Heeger, 2007; Turner, 2001; 2002) thus limiting the spatial specificity of BOLD. In addition, the BOLD response will depend on the vascular density, which varies between brain areas (Vigneau-Roy, Bernier, Descoteaux, \& Whittingstall, 2013), and might be compromised in conditions such as stroke. The neurovascular coupling also imposes constraints on the temporal resolution of the BOLD signal. The haemodynamic response function (HRF) that defines BOLD signal change is characterised by 1-2 second delay between the onset of neural activity and the time required for blood to travel to the active region, and a time to peak of approximately 6-12 seconds, with large inter-subject variations. A method able to combine the high spatial resolution of MRI with a more direct measurement of brain activity would offer attractive advantages. As cell swelling has been reported as one of the physiological processes accompanying neuronal activation (Tasaki \& Iwasa, 1982b; 1982a), diffusion-weighted (DW) MRI has been proposed as a potential candidate for detecting temporal patterns in cell physiology (Aso et al., 2009; Latour, Hasegawa, Formato, Fisher, \& Sotak, 1994; Le Bihan et al., 2012; Le Bihan, Urayama, Aso, Hanakawa, \& Fukuyama, 2006).

\section{BACKGROUND}

The idea that DW MRI could be sensitive to functional activation has been mooted for several years (le Bihan et al. 2012). Initial attempts to exploit this sensitivity built upon the concept of intravoxel incoherent motion (IVIM) reflecting the displacement of water molecules in the pseudo-randomly orientated capillary network at low b-values (less than 100-200 s/ $\mathrm{mm}^{2}$ ) (Turner et al., 1990). The observation of a transient reduction in $A D C$ of the occipital cortex during visual stimulation at high b value $\left(\sim 1400 \mathrm{smm}^{-2}\right)$, however, pointed at a different mechanism (Darquié, Poline, Poupon, Saint-Jalmes, \& Le Bihan, 2001). This was initially interpreted as a consequence of cortical cell swelling resulting in increased tortuosity of the extracellular space. In 2006, LeBihan et al (Le Bihan et al., 2006) explored the changes in ADC occurring at a range of $b$-values, demonstrating that the effect is enlarged at large $b$-values $\left(>1500 \mathrm{smm}^{-2}\right)$ and seems to precede the haemodynamic response measured by blood oxygenation level dependent (BOLD) contrast. Based on this observation they advanced the hypothesis that changes in DW MRI reflect early biophysical events associated with neuronal activation and thus provide a more direct measure than BOLD that, as discussed above, has a large contribution from large and distant draining veins.

Le Bihan et al (2006) proposed a quantitative interpretation by means of the biphasic model of water diffusion in the gray matter: according to such model, the empirically observed slow (SDP) and fast (FDP) diffusion pools correspond to both, extra and intracellular spaces, with SDP originating from molecules 
hindered by their close proximity to cell membranes (Le Bihan et al., 2006). Swelling of the cell membrane may thus result in the expansion of the SDP volume fraction (and a consequent reduction in the FDP volume fraction) resulting in a decrease in water diffusion. Fitting this model to the data from 6 participants yielded a constant increase of approximately $0.6 \%$ in the SDP volume fraction. This model, however, is in conflict with the extremely fast exchange rates expected between these water fractions (Cowan et al., 2005; Jungwirth, 2011).

The hypothesis that the diffusion $\mathrm{fMRI}$ signal originates solely from neuronal activity has, been challenged by other authors. Miller et al. (Miller et al., 2007) used hypercapnia, a condition known to induce vascular changes measurable with BOLD, in the absence of neuronal signal. They showed that the amplitude of the change in diffusion fMRI was comparable to that obtained with a visual stimulation, and failed to replicate the precedence of the diffusion $\mathrm{FMRI}$ response to stimulation compared to BOLD. Other studies have also failed to replicate the b-factor dependency (Autio \& Roberts, 2014). Finally, a certain degree of anisotropy of the hypercapnia-induced signal changes has been reported, which makes it less likely to be related to cell swelling in the grey matter (Miller et al., 2007). As an alternative explanation, Pampel et al (Pampel, Jochimsen, \& Möller, 2010) have suggested that background gradients resulting from the difference in magnetic susceptibility between water and blood might interact with the diffusion gradients thus causing the observed changes in ADC.

\section{THE PROMISE}

The exact source of diffusion $\mathrm{fMRI}$ contrast has yet to be determined. Part of the difficulty in designing appropriate experiments lies in the inherently lowSNR of the diffusion fMRI signal, which limits the range of feasible experimental manipulations. Such a low SNR is one of the biggest concerns about diffusion fMRI, as even the simplest experiment requires the acquisition of very large datasets (R. J. Williams, Reutens, \& Hocking, 2016) The advent of faster sequences such as multiband imaging, aka simultaneous multi-slice (Moeller et al., 2010), can help, but a significant boost in SNR is the only way to make these experiments practical. Moving to higher field strength increases the $b=0$ signal, but due to the shortening of $T 2$ yields a limited benefit at high b-values. A real improvement can only be achieved with strategies that allow shorter TEs to be used with heavily diffusion-weighted sequences, thus mitigating the signal loss due to T2 decay. Multiple channel receiver coils (e.g., 128-channels), with their better SNR compared to 20- or 32-channel coils, could help, especially if combined with parallel imaging schemes that reduce the TE. However, the acceleration factor achievable is limited (Larkman and Nunes, 2007). The use of ultra-large gradients is thus an efficient way of increasing the SNR in DW-MRI. In terms of clarifying the source of the diffusion fMRI signal change, a high gradient performance-scanner can help by addressing 4 fundamental issues: 


\section{Is the diffusion signal change associated with functional activation dependent on the $b$-value?}

Le Bihan et al. (Le Bihan et al., 2006) reported a linear increase of the raw signal change with $b$, for $b$ ranging from 600 to $2400 \mathrm{~s} / \mathrm{mm}^{2}$. They argued this was compatible with the biphasic model, as higher $\mathrm{b}$ values would supress more of the FDP signal and therefore result in the signal being more heavily weighted by SDP. As discussed in Section 4 on multi-contrast experiments, ultra-high gradients allow much higher $b$ values than possible on standard MRI scanners, while keeping the TE relatively short. Based on Fig 1, the same $b$ value of $4000 \mathrm{smm}^{-2}$ (which is close to the optimum recommended for diffusion $\mathrm{fMRI}$ ) can be achieved with a TE of $70 \mathrm{~ms}$ on a "super-scanner", compared to a TE of $120 \mathrm{~ms}$ on a system with maximum gradient strength of $80 \mathrm{mTm}^{-1}$. This results in 2.3 times large SNR for a T2 of $60 \mathrm{~ms}$. Using the theoretical relationship between temporal SNR and number of points needed (Murphy et al., 2007), we can infer that, in order to detect the expected effect size of $0.16 \%$ (Le Bihan et al., 2006), the number of sampling points can be reduced by a factor of 12 when using ultra-strong gradients. The faster gradients also allow efficient implementations of high b-value twice-refocused spin echo sequences (Reese, Heid, Weisskoff, \& Wedeen, 2003), which should mitigate the impact of BOLD background gradient contributions (Pampel et al., 2010) to the diffusion $\mathrm{fMRI}$ signal.

\section{Is the diffusion signal change associated with functional activation dependent on the TE?}

Another interesting question is whether the change observed with activation in the diffusion fMRI raw signal is caused not by a change in the SDP compartment size, as expected if the effect was related to cell swelling, but by a change in the $\mathrm{T} 2$ of the diffusion coefficient of either compartment. Miller et al hypothesised that veins and capillaries might have different T2 BOLD effects (Miller et al., 2007) because of their differing degree of oxygenation, which might explain the changes in the raw signal.

Access to ultra-high magnetic field gradients allows large b-values to be obtained at relatively short TEs, and therefore the effects of mapping the TE dependency of the raw signal (see also Section 4 on multicontrast experiments).

\section{Is the diffusion signal change associated with functional activation isotropic?}

If the effect observed with diffusion $\mathrm{fMRI}$ is caused by swelling of the neuronal cell bodies, it would be expected to be fairly isotropic, i.e., independent of the direction of the diffusion gradients. However, some anisotropy has been observed (Miller et al., 2007) potentially attributable to signal coming from structures with ordered geometry, such as vessels in the cortex. Higher resolution scans, achievable through the SNR enhancement granted by ultra-high gradients (see Section 5) could help to clarify the origin of this observed anisotropy. Realistically, however, the estimated SNR advantage of 2.3 estimated for T2=60ms at $\mathrm{b}=400 \mathrm{~s} / \mathrm{mm}^{2}$ allows a reduction in isotropic voxel size of approximately 0.75 .

\section{Is the diffusion signal change associated with functional activation more spatially specific than BOLD?}

One of the observations that support the neuronal origin of the diffusion $\mathrm{fMRI}$ signal is the more precise localisation of activations within the cortical ribbon, compared to BOLD (Le Bihan et al., 2006). However, 
due to the extremely low SNR of diffusion $\mathrm{fMRI}$, smoothing is necessary to enable the measurement of any activation at all, thus compromising the spatial resolution (Williams et al., 2016).

As ultra-high magnetic field gradients enable diffusion measurements with short TE, the resulting enhanced SNR could be spent on investigating the spatial specificity of diffusion fMRI. Ideally, the profiles of diffusion and BOLD fMRI could be compared between different cortical layers (Williams et al., 2016).

\section{IMAGE QUALITY TRANSFER}

\section{THE CHALLENGE AND ITS IMPORTANCE}

In this article we have identified clearly the gains to be made by investing in ultra-strong diffusion gradient technology. However, as devices with this technology are not mass-produced, they incur considerable cost and are not currently widely available. A reasonable question, when reading an article such as this would be: "That's all very well, if you have access to such high performance equipment. However, most clinical/ research imaging facilities do not. How then, can such technology be of widespread benefit?"

One approach is to establish regional centres that are willing to accept patients / host research visits from parties outside of the host institution (this is the model employed in establishing the National Facility for In Vivo MR Imaging of Human Tissue Microstructure in the UK). However, this still necessitates the establishment of sufficient regional centres to provide access. Another approach is to 'transfer' the benefits of ultra-strong gradient technology to systems with lower gradient performance. The idea of image quality transfer (IQT) is to use modern machine learning techniques to construct a mapping from "low quality" to "high quality" images from matched pairs. We can then use the learned mapping to approximate the high quality equivalent of a low quality image, i.e., estimate the image we would have obtained from more time-expensive protocol or hard-to-access imaging device using only data from easily accessible imaging hardware and software.

Through this technology, we can potentially bridge the gap between rare and experimental systems, such as ultra-strong gradient or ultra-high-field MRI scanners, and widely available commercial systems and bring the power of tomorrow's imaging technology into today's clinical applications. The existence of oneoff bespoke devices such as the microstructure scanner enables this kind of approach. The ambitious potential is for a new medical-imaging "paradigm exploiting coupled design of a) bespoke high-powered devices to capture databases of high quality images, and b) widely deployed cheap and/or low-power devices designed specifically to exploit the rich information from (a)" via IQT (Alexander et al. 2017).

\section{BACKGROUND}


Early demonstrations of the idea show good promise. Alexander et al. (Alexander, Zikic, Ghosh, Tanno, Wottschel, et al., 2017b; Alexander, Zikic, Zhang, Zhang, \& Criminisi, 2014) show two potential applications in dMRI: i) resolution enhancement of low-resolution diffusion-parameter maps to high resolution; and ii) estimation of microstructural maps from diffusion tensor images. The resolution enhancement from learned mappings shows substantial improvements over standard techniques, such as interpolation. One key result demonstrates how the operation enables tractography to recover thin white matter fibre pathways, previously recoverable only at $1.25 \mathrm{~mm}$ isotropic resolution (unobtainable in standard clinical protocols) but from data acquired at $2.5 \mathrm{~mm}$ isotropic resolution (see Figure 10a).

\section{FIGURE 10 NEAR HERE}

Figure 10. Examples of image quality transfer from the literature. a. recovering thin white matter fibre pathways, previously recoverable only at $1.25 \mathrm{~mm}$ isotropic resolution (unobtainable in standard clinical protocols) from data acquired at $2.5 \mathrm{~mm}$ isotropic resolution (Alexander et al. 2017b); b. Constructing a mapping from $3 T$ structural images to 7 T equivalents (Bahrami et al. 2016)

Moreover, the operation shows remarkable generalizability beyond training data - even mappings learned from fixed ex vivo monkey brain images enhance in vivo human brain images substantially better than standard methods. The second operation - estimating microstructural maps from diffusion tensor imaging - is challenging, because microstructural maps, e.g. from NODDI (Zhang et al., 2012) and spherical mean technique (Kaden et al., 2016) typically require multi-shell acquisition protocols whereas DT-MRI requires a much less rich, and cheaper, acquisition protocol over only one shell. The potential of IQT is to estimate these microstructural maps from historical single-shell data sets, from which it would normally be impossible to compute such maps. However, although the estimated microstructural maps in (Alexander, Zikic, Ghosh, Tanno, Wottschel, et al., 2017b) broadly match ground truth from multi-shell data sets, significant detail is lost and the technology likely needs further development before the idea will find useful application. Golkov et al. (Golkov et al., 2016) used a similar idea to learn a prior on the structure of NODDI maps that enables reconstruction from sparse data sets, although still involving multiple b-values. Bahrami et al. (Bahrami et al. 2016) aim to construct a mapping from 3T structural images to 7T equivalents again showing promising results (see Figure 10b). The original implementation of Alexander's work uses patchbased, random-forest regression; Bahrami's 7T-3T mappings use canonical correlation analysis. A more recent implementation for diffusion resolution enhancement (Tanno et al., 2017) exploits the power of deep learning to gain substantial performance enhancements. Bahrami's more recent work also explores deep neural networks for mapping 3T to 7T (Bahrami et al. 2016b).

\section{THE PROMISE}


Image quality transfer remains in its infancy and substantial further development and validation is necessary to support widespread adoption. Key challenges in development include: finding matched pairs, and handling and quantifying uncertainty in mappings. One way to find matched pairs is to acquire separate low and high-quality images (Bahrami et al., 2016). However, this approach leads to issues of image registration, as alignment is never perfect, while learning approaches typically assume it is. Future efforts may consider embedding misalignment implicitly in the learning process. Another approach, (Alexander, Zikic, Ghosh, Tanno, Wottschel, et al., 2017b), is to simulate the low quality image from the high quality image through a sub-sampling procedure. While this, by definition, produces pairs of images that are in perfect alignment, the artificial construction of the low-quality image can reduce the accuracy of the learned mapping. Quantification of uncertainty is relatively straightforward with traditional learning algorithms, such as random forests, and Tanno et al. (Tanno et al., 2016) showed compelling results in error prediction from IQT through a Bayesian random forest implementation. Uncertainty estimation with deep neural networks is more challenging. Although efforts to do so show promise (Tanno et al., 2017), this is still an area for significant future research.

\section{PRACTICAL ISSUES FOR THE USE OF ULTRA-STRONG GRADIENTS}

The advantages of ultra-strong gradients for diffusion-based microstructure imaging have been clearly outlined in the previous sections. Here, we discuss the practical issues that set limits on the exploitation of ultra-strong gradients for different types of microstructure imaging studies. The Connectom scanner's ultra-strong gradient hardware can reach a maximum gradient amplitude of $300 \mathrm{mT} / \mathrm{m}$ (McNab et al., 2013). The maximum achievable slew-rate is $200 \mathrm{mT} / \mathrm{m} / \mathrm{ms}$, which is comparable with the maximum slew rate of other commercially available gradient systems that provide lower maximum gradient strengths. However, while these specifications promise vastly improved gradient performance in terms of achievable b-value and q-value per unit TE, it is currently not possible to exploit them to the full hardware limits due to physiology-related constraints.

\section{Physiological limits: Peripheral Nerve Stimulation (PNS) and Cardiac Stimulation:}

The first relevant safety constraint concerns the avoidance of peripheral nerve stimulation (PNS). The timevarying magnetic fields produced by fast-switching gradient systems can induce stimulation in peripheral nerves. This is experienced as a tingling or twitching sensation close to the threshold for stimulation, but can become uncomfortable or painful as the rate of change of field is increased significantly above threshold. The threshold for stimulation depends on the spatio-temporal characteristics of the electric fields induced in the conducting tissues of the body and their relationship to the anatomical and physiological characteristics of peripheral nerves. Although this complexity makes it difficult to predict the 
site and threshold of stimulation at the individual subject level (Davids, Guérin, Malzacher, Schad, \& Wald, 2017) the likelihood of stimulation depends upon: (i) the rate of change of gradient amplitude with time; (ii) the peak gradient amplitude; and (iii) the position of the subject within the gradient coils (Chronik \& Rutt, 2001). To characterise the average stimulation characteristics of a gradient system, MR scanner manufacturers generally test the PNS limits in experiments on human subjects using controlled gradient waveforms. Using this experimental data, the threshold for stimulation for other gradient waveforms can then be well characterized using the SAFE model (Hebrank \& Gebhart, 2000) and thus PNS can be controlled. Rapidly time-varying magnetic field gradients can also potentially cause cardiac stimulation. Based on studies using direct electrical stimulation of the heart, this would be expected to take the form of ectopic beats and induced cardiac arrhythmia at lower levels of stimulation and fibrillation at higher stimulation levels. No such cardiac stimulation due to MRI gradient switching has ever been reported in human subjects, though ectopic beats have been generated in animal experiments using small coils to produce extremely high rates of change of magnetic field (2700 T/s over a ramp duration of $530 \mu \mathrm{s})$ (Bourland, Nyenhuis, \& Schaefer, 1999; Schaefer, Bourland, \& Nyenhuis, 2000). As a result of differences in the electrophysiology of cardiac muscle and peripheral nerves, the probability for cardiac stimulation arising from gradient waveforms on clinical scanners is extremely small (of the order of $10^{-9}$ ), but the higher hardware capabilities of the Connectom scanner means that the potential to cause cardiac stimulation must be considered. In previous work, (Setsompop et al., 2013) it was also found that magnetophosphenes were perceived by some subjects when the eyes were significantly displaced from the scanner's iso-centre and strong time-varying gradients with long rise times (> $130 \mathrm{mT} / \mathrm{m}$ with $5-7 \mathrm{~ms}$ risetime) were applied. These flashes of light in the peripheral visual field are thought to result from direct stimulation of the retina, and will need to be given some consideration in studies where the head is not situated close to iso-centre.

The PNS limitations on the Connectom gradients when the subject has their head at iso-centre are shown in Figure 11. It shows stimulation limit plots for $G x$.

FIGURE 11 NEAR HERE

Figure 11. Comparison of performance limitations imposed by the system and human physiology. The peripheral nerve and cardiac stimulation thresholds for the Connectom AS302 gradient as a function of gradient strength and rise time are plotted. Up to around $180 \mathrm{mT} / \mathrm{m}$, the PNS limitations dictate the gradient performance. However, above $180 \mathrm{mT} / \mathrm{m}$, the theoretical possibility of cardiac stimulation dominates the gradient performance. These limitations are built into the scanner through the SAFE model (Hebrank \& Gebhart, 2000) and hardware gradient safety watchdog. Safe operation of the scanner is always ensured by these mechanisms.

For peak gradient strengths less than $\sim 80 \mathrm{mT} / \mathrm{m}$, on a single axis, (the $X$-axis) it is possible to utilise the maximum, $200 \mathrm{mT} / \mathrm{m} / \mathrm{ms}$ slew rate allowed by the hardware without causing PNS. For larger gradient 
strengths, and particularly when using combinations of gradients on 2 or 3 axes, the slew rate must be limited to avoid PNS, with a limiting slew rate of around $150 \mathrm{mT} / \mathrm{m} / \mathrm{ms}$ for the maximum accessible gradient strength of $300 \mathrm{mT} / \mathrm{m}$. For the single gradient axis in Figure 11, above $150 \mathrm{mT} / \mathrm{m}$ the gradient slew rate must be further reduced to conform to the IEC limit for cardiac stimulation, which is based on the predicted probability of cardiac stimulation. This means that a rise time of $\sim 3.6 \mathrm{~ms}$ is needed to reach the full gradient amplitude of $300 \mathrm{mT} / \mathrm{m}$, corresponding to a slew rate of around $80 \mathrm{mT} / \mathrm{m} / \mathrm{ms}$. While this restriction may not adversely affect classical Stejskal-Tanner diffusion experiments, in which the duration of the pulses are relatively long compared to the rise times, it is clear that this places us far from the 'narrow pulse' approximation in q-space imaging, and the achievable spectral bandwidth in temporal diffusion spectroscopy experiments (e.g.(Gore et al., 2010)) will be severely affected when using the highest gradient amplitudes. It is important to note however that although the limits set on gradient waveforms to avoid PNS are based on experimental measurements on human subjects, the cardiac stimulation limit is based on inference and extrapolation from animal experiments, (Bourland et al., 1999; Reilly, 1995; Reilly, 1989; 1991) that have led to the maximum rate of change of magnetic field at the heart being limited to values less than $20 /\left(1-e^{-\frac{t_{s, e f f}}{3}}\right) \mathrm{T} / \mathrm{s}$, where $t_{s, e f f}$ is the stimulus duration in ms. (Medical electrical equipment Part 2-33: Particular requirements for the safety of magnetic resonance equipment for medical diagnosis IEC 60601-2-33:2002).

This can be translated to limits on the gradient strength and slew rate by estimating the position of the heart within the gradient coil-set and knowledge of the spatial variation of the gradient fields. As the cardiac stimulation limit becomes the main constraint on gradient performance it would be timely to revisit the prior literature and to plan new simulations (Davids et al., 2017) and experiments to test the assumptions on which the limits have been based (though acknowledging that any such experiments are likely to be challenging and complex). These investigations are well worth considering, since were the operating limit set by the PNS, rather than cardiac stimulation, limit, the time needed to ramp to a field gradient of $300 \mathrm{mT} / \mathrm{m}$ in the Connectom system could be reduced to around $2 \mathrm{~ms}$.

Modification of the designs of whole-body gradient coil systems of the kind employed in the Connectom system specifically to reduce the likelihood of nerve stimulation in particular body regions may also be considered in the future (Davids et al., 2017; Hidalgo-Tobon, Bencsik, \& Bowtell, 2011). Use of head-only gradient coils can provide access to higher gradient performance than whole-body sized systems, like the Connectom gradients, at the threshold for stimulation, since the reduced coil size general limits the maximum $\mathrm{dB} / \mathrm{dt}$ values produced in the body. For example, a recently described asymmetric head gradient coil system was shown to be able to exploit the full hardware capability in slewing to a maximum gradient of $80 \mathrm{mT} / \mathrm{m}$ at a rate of $700 \mathrm{mT} / \mathrm{m} / \mathrm{ms}$ for the y (anterior-posterior) gradient direction without causing PNS (in 22/24 subjects scanned) (Lee et al., 2016). However, head-only gradient coils that can generate 
gradient strengths as high as $300 \mathrm{mT} / \mathrm{m}$ have, not yet been demonstrated and it is worth noting that the concomitant field effects (described below) are generally much more severe in asymmetric gradient sets. Moreover, head-only gradient sets limit the available space for radiofrequency coils, which becomes problematic for systems larger numbers of receiver coils (e.g., more than 32 ) and for multi-channel transmit systems.

\section{Duty cycle/heat dissipation:}

The Connectom gradients have been designed to operate with the following duty-cycle limits (assuming Trapezoidal gradient pulses)- at $300 \mathrm{mT} / \mathrm{m}$ : $6 \%$ on all axes or $17 \%$ on one axis, at $210 \mathrm{mT} / \mathrm{m}$ : $12 \%$ on all axes or $35 \%$ on one axis, at $90 \mathrm{mT} / \mathrm{m}: 50 \%$ on all axes or $100 \%$ on one axis.

Although the diffusion gradient pulses typically have a short duration, when used at maximum gradient strength, the $6 \%$ limit on duty cycle can increase the required TR substantially, especially if the EPI train is short or non-EPI readout techniques are used. Power amplifiers generally operate with maximum and average power specifications. They are designed for average power at $100 \%$ duty cycle, but can also be used at peak power with limited duty cycle. The same holds true for gradient coils, for which the limiting factor is power dissipation in the electrical conductors. The most efficient design of ultra-strong gradient kit uses all available space (i.e., wire cross section) in the magnet bore for gradient field-generating conductors and cooling layers. In the Connectom system (McNab et al., 2013) the optimum under these technical boundary conditions requires the use of four gradient amplifiers and results in a nominal (or average) gradient amplitude of $\sim 100 \mathrm{mT} / \mathrm{m}$. A further increase under these conditions (i.e., limited space in the magnet bore) would require new cooling technologies for the gradient coil and an increase of the average power of the amplifier. Thinking to the future, one possibility might be to incorporate a superconducting gradient coil either within the magnet cryo vessel or within a separate cryo vessel, keeping in mind the critical field strength for superconductivity which depends on the static $B_{0}$ field and the time varying magnetic field of the gradient field conductors. Although this is a challenge given that the currently available wire materials show significant heat production for alternating currents in the $1-5 \mathrm{KHz}$ range even at cryogenic temperatures, latest research seem to indicate that this is technically achievable (see: Yuan and Shen, 2007 and patent number GB2433600)

\section{Gradient non-linearity:}

A current trade-off in achieving ultra-high gradient amplitudes has been in the region over which the gradient system behaves linearly. A strict boundary condition for linearity would lead to suboptimal gradient efficiency and lower the PNS limit. Thus, the system was designed to give linearity over the typical volume of a head, with homogeneity of approximately $6 \%$ over a $20 \mathrm{~cm}$ sphere and $17 \%$ over a $40 \mathrm{~cm}$ 
sphere. However, due to the nature of the gradient design (a symmetric, whole body system), such a system can be applied to other parts of the human body, but the reduced linearity over the extended volume require improved strategies for handling gradient non-linearity. The impact of non-linearity in terms of image distortion is well documented (Jovicich et al., 2006) and scanner vendors typically correct these distortions using well-established unwarping algorithms. Since the non-linearities in typical wholebody scanners in a volume of the size of human head are very small, the effects of such non-linearities are very minor. The gradient non-linearities can have a significant impact on diffusion studies in ways other than simple image distortion, if positioning is not well controlled. Depending on the non-linearity, the bmatrices at each voxel location in the brain will be spatially dependent and differ from the nominal values (Bammer et al., 2003; Borkowski, Kłodowski, Figiel, \& Krzyżak, 2017; Malyarenko, Ross, \& Chenevert, 2014; Mohammadi et al., 2012). Besides, gradient non-linearity distorts the true image in concert with $B_{0}$ inhomogeneity, making it harder to disentangle the two effects in a sequential manner at the postprocessing stage. This complication has led to the development of diverse processing pipelines (Fan et al., 2016; Glasser et al., 2013; Setsompop et al., 2013; Sotiropoulos et al., 2013). Moreover, given that the gradient non-linearities need not be rotationally symmetric, head movement can complicate the b-matrix temporal evolution at different voxel locations significantly, requiring one to keep a temporal history of bmatrix evolution at each location to remove unwanted biases in measured diffusion parameters. The effect of each of these issues on the final microstructure parameters obtained should be carefully assessed and where necessary, dedicated processing pipelines that address gradient non-linearity should be implemented to make the best use of these novel hardware platforms.

\section{Concomitant Fields}

The use of high gradient strengths increases the concomitant field effects (Bernstein et al. 1998; Baron, Lebel, Wilman, \& Beaulieu, 2012; Meier et al. 2008; Norris \& Hutchison, 1990) that can confound diffusion measurements. Concomitant fields arise because the vector magnetic field generated by a gradient coil has to satisfy Maxwell's equations. Hence, to ensure that the curl and divergence of the field are both zero in current free regions of space, the desired spatial variation of the z-component of the magnetic field must always be accompanied by a spatially varying $x$-and/or $y$-component of the field. Since these concomitant fields are orthogonal to (and much smaller in magnitude than) $B_{0}$, their effect on the Larmor frequency can usually be neglected, but in fact there is always a net change to the Larmor frequency $\omega_{c}=\gamma B_{c}$ due to these concomitant fields which is given by

$$
B_{C}=\frac{\left(G_{x}^{2}+G_{y}^{2}\right) z^{2}}{2 B_{0}}+\frac{G_{z}^{2}\left(x^{2}+y^{2}\right)}{8 B_{0}}-\frac{G_{x} G_{z} x z}{2 B_{0}}-\frac{G_{y} G_{z} y z}{2 B_{0}},
$$

when gradients $G_{x}, G_{y}$ and $G_{z}$ are applied simultaneously. Equation [1] shows that concomitant fields scale quadratically with the applied gradient strength and so become more than 56 times larger when the gradient strength is increased from $40 \mathrm{mT} / \mathrm{m}$ (gradients on a conventional clinical scanner) to $300 \mathrm{mT} / \mathrm{m}$ 
whilst keeping $B_{0}$ fixed. Note that this problem would be even more pronounced for asymmetric gradient designs (e.g., head-only gradient systems), the extra complication arising from the presence of odd-order terms to the concomitant field correction. Techniques to circumvent such problems have been proposed, including manipulating the pre-emphasis (Tao et al. MRM 2017), real-time adjustment of the centre $\left(B_{0}\right)$ frequency (Weavers et al. MRM 2018). These concomitant field effects are well known to cause image distortion, which particularly affects echo planar imaging since the concomitant fields due to the switched read-out gradient produce a progressively increasing position-dependent phase accumulation down the echo train (Y. P. Du, Joe Zhou, \& Bernstein, 2002; Norris \& Hutchison, 1990). Fortunately, with knowledge of the applied gradients this distortion can be corrected (Du et al., 2002). When the Connectom gradient system is applied in diffusion imaging, the peak gradient strength during the EPI readout is similar to that used on a conventional scanner, so the problem of image distortion due to concomitant fields is not particularly exacerbated, but the effects of the concomitant fields due to the diffusion encoding gradient pulses can be challenging. In particular, the quadratic dependence of the effective field, $B_{c}$, on the gradient strength means that phase accumulation due to concomitant fields is identical for positive and negative gradient lobes of the same amplitude and duration. There is consequently no net phase accumulation when unipolar gradients are applied in conjunction with a $180^{\circ} \mathrm{RF}$ pulse in a Stejskal-Tanner sequence (Stejskal \& Tanner, 1965), but the concomitant fields produce a spatial phase variation over the imaging region when balanced bipolar gradient pulses are applied. This is problematic for diffusion encoding sequences employing bipolar gradients (Setsompop et al., 2013) such as the twice-refocused spin echo sequence (Reese et al., 2003) that is commonly used to reduce eddy current effects With such sequences, throughslice variation of the accrued phase can produce a spatially varying image attenuation which modulates the effect of diffusion, while the variation of phase within the image plane can shift the time of acquisition of the centre of k-space by different amounts for different regions causing image blurring and signal loss (Baron et al., 2012). The terms in Eq. [2] varying as $x z$ and $y z$ can also produce a linear phase shift across the whole image, which may shift the centre of $k$-space outside of the acquisition window, thus causing complete signal loss (Baron et al., 2012). For example if a bipolar x-gradient of $300 \mathrm{mT} / \mathrm{m}$ strength is applied with lobes of $10 \mathrm{~ms}$ duration, the phase variation across a $2 \mathrm{~mm}$-thick axial slice displaced by $2.5 \mathrm{~cm}$ from isocentre is around $8 \pi$, while the linear phase variation produced in the same axial slice when bipolar gradient encoding of similar strength and duration is applied with the gradient oriented at $45^{\circ}$ to $\mathrm{x}$ and $\mathrm{z}$ would be around $320 \pi \mathrm{m}^{-1}$ which would be sufficient to shift the centre of $k$-space outside the acquisition window in $3 \mathrm{~mm}$ resolution EPI data. Both these values increase linearly with the displacement of the axial slice from isocentre. While these effects mean that there are strong incentives to stick with ST sequences for diffusion encoding when using ultra-strong gradients, approaches for ameliorating the effects of concomitant fields in sequences using bipolar gradients have been developed (Baron et al., 2012; Meier, Zwanger, Feiweier, \& Porter, 2008; Baron et al., 2012). These approaches, which involve applying compensating pre-phasing gradient pulses to eliminate the spatially varying phase produced by the 
concomitant fields during diffusion encoding, currently allow localised reduction of confounding effects, but their efficacy is yet to be evaluated with ultra-high gradient strengths. Full exploitation of the power of Connectom-like gradients in experiments using bipolar gradients will require the development of new approaches that can cancel the phase variation due to concomitant fields over larger image volumes.

\section{CONCLUSION}

Even though diffusion encoding with strong gradients raises challenges, it is without question that with ongoing developments to better understand such issues, a microstructural 'super-scanner' provides considerable improvements to 'conventional' diffusion measurements and facilitates new measurements that are impractical at lower gradient amplitudes. New insights arising from this extended measurement space, together with practical experience on the challenges related to strong gradients, will provide a sound foundation to develop the next generation of microstructural super-scanners.

\section{ACRONYMS USED IN THIS WORK:}

$A D C=$ apparent diffusion coefficient

AMICO = Accelerated Microstructure Imaging via Convex Optimization

BOLD = blood-oxygenation level-dependent

CHARMED = composite hindered and restricted model of diffusion

CPMG = Carr-Purcell-Meiboom-Gill

DDE $=$ double diffusion encoding

DIVIDE = diffusional variance decomposition

DKI = diffusion kurtosis imaging

$\mathrm{dMRI}=$ diffusion $\mathrm{MRI}$

$\mathrm{DTI}=$ diffusion tensor imaging

DW-MRS = Diffusion-weighted Magnetic Resonance Spectroscopy

DW-SSFP = diffusion weighted steady-state free precession;

DW-STE $=$ diffusion weighted stimulated echo

EPSRC $=$ Engineering and Physical Sciences Research Council

$F A=$ fractional anisotropy

FEXI = Filter Exchange Imaging

gSlider-SMS = Generalised Slice Dither Enhanced Resolution with Simultaneous Multislice

HARDI = high angular resolution diffusion imaging

IMPULSED = imaging microstructural parameters using limited spectrally edited diffusion

IQT = image quality transfer

IR-PGSE = inversion-recovery prepared pulsed-gradient spin echo 
$M D=$ mean diffusivity

$\mathrm{MDE}=$ multiple diffusion encoding

$\mathrm{NAA}=\mathrm{N}$-acetyl-aspartate

NODDI = neurite orientation dispersion and density imaging

OGSE = oscillating gradient spin echo

POMACE $=$ Pulsed and oscillating gradient MRI for assessment of cell size and extracellular space

PGSE $=$ pulsed gradient spin echo

PGSTE $=$ pulsed-gradient stimulated echo

PNS = peripheral nerve stimulation

PRESS $=$ point resolved spectroscopy

$\mathrm{QTE}=\mathrm{Q}$-space trajectory encoding

SAFE = Stimulation approximation by filtering and evaluation

$\mathrm{SDE}=$ single diffusion imaging

SNR = Signal to noise ratio

$\mathrm{TE}=$ echo time

$\mathrm{tCr}=$ total Creatine

tCho $=$ total Choline

VERDICT $=$ Vascular, Extracellular, and Restricted Diffusion for Cytometry in Tumours

\section{ACKNOWLEDGEMENTS:}

The UK National Facility for In Vivo MR Imaging of Human Tissue Microstructure was generously funded by the Engineering and Physical Sciences Research Council (EPSRC) (grant EP/M029778/1), and The Wolfson Foundation, to whom the authors give their thanks.

DKJ is supported by a Wellcome Trust Investigator Award (096646/Z/11/Z) and a Wellcome Trust Strategic Award (104943/Z/14/Z). CMWT is supported by a Rubicon grant (680-50-1527) from the Netherlands Organisation for Scientific Research (NWO) and Wellcome Trust grant (096646/Z/11/Z). MP is supported by EPSRC (EP/G007748, EP/I027084/01, EP/L022680/1, EP/M020533/1, N018702). EPSRC grants EP/G007748 EP/L022680/1 EP/M00855X/1 EP/M020533/1 EP/N018702/1 EP/R014019/1 and MRC grant MR/M009106/1 support DCA's work on this topic.

The authors are indebted to Dr. Ralph Kimmlingen, Siemens Healthcare GmbH, Erlangen, Germany for his helpful comments and contributions to Section 11 of this article, and to Dr Kawin Setsompop, Athinoula A. Martinos Center for Biomedical Imaging, for supplying the diffusion-weighted data used to create Figure 6. 


\section{REFERENCES}

Aboitiz, F., Scheibel, A. B., Fisher, R. S., \& Zaidel, E. (1992). Fiber composition of the human corpus callosum. Brain Research, 598(1-2), 143-153.

Aboitiz F, Montiel J. (2003). One hundred million years of interhemispheric communication: the history of the corpus callosum. Braz J Med Biol Res. 36(4):409-20.

Aggarwal M, Zhang JY, Pletnikova O, Crain B, Troncoso J, Mori S. Feasibility of creating a high-resolution 3D diffusion tensor imaging based atlas of the human brainstem: A case study at 11.7 T. Neurolmage. Jul 1 2013;74:117-127.

Aggarwal M, Nauen DW, Troncoso JC, Mori S. Probing region-specific microstructure of human cortical areas using high angular and spatial resolution diffusion MRI. Neurolmage. Jan 15 2015;105:198-207.

Ahlgren, A., Knutsson, L., Wirestam, R., Nilsson, M., Ståhlberg, F., Topgaard, D., \& Lasič, S. (2016). Quantification of microcirculatory parameters by joint analysis of flow-compensated and non-flowcompensated intravoxel incoherent motion (IVIM) data. NMR in Biomedicine, 29(5), 640-649. http://doi.org/10.1002/nbm.3505

Alexander DC. 2008. A general framework for experiment design in diffusion MRI and its application in measuring direct tissue-microstructure features. Magn Reson Med. 2008 Aug;60(2):439-48. doi: $10.1002 / \mathrm{mrm} .21646$.

Alexander, D. C., Dyrby, T. B., Nilsson, M., \& Zhang, H. (2017a). Imaging brain microstructure with diffusion MRI: practicality and applications. NMR in Biomedicine. http://doi.org/10.1002/nbm.3841

Alexander, D. C., Hubbard, P. L., Hall, M. G., Moore, E. A., Ptito, M., Parker, G. J. M., \& Dyrby, T. B. (2010). Orientationally invariant indices of axon diameter and density from diffusion MRI. Neurolmage, 52(4), 1374-1389. http://doi.org/10.1016/j.neuroimage.2010.05.043

Alexander, D. C., Zikic, D., Ghosh, A., Tanno, R., Wottschel, V., Zhang, J., et al. (2017b). Image quality transfer and applications in diffusion MRI. Neurolmage, 152, 283-298. http://doi.org/10.1016/j.neuroimage.2017.02.089

Alexander, D. C., Zikic, D., Zhang, J., Zhang, H., \& Criminisi, A. (2014). Image Quality Transfer via Random Forest Regression: Applications in Diffusion MRI. In Medical Image Computing and Computer-Assisted Intervention - MICCAI 2014 (Vol. 8675, pp. 225-232). Cham: Springer, Cham. http://doi.org/10.1007/978-3-319-10443-0_29

Andrews, D. S., Avino, T. A., Gudbrandsen, M., Daly, E., Marquand, A., Murphy, C. M., et al. (2017). In Vivo Evidence of Reduced Integrity of the Gray-White Matter Boundary in Autism Spectrum Disorder. Cerebral Cortex (New York, N.Y. : 1991), 27(2), 877-887. http://doi.org/10.1093/cercor/bhw404

Andrews, T. J., Osborne, M. T., \& Does, M. D. (2006). Diffusion of myelin water. Magnetic Resonance in Medicine, 56(2), 381-385. http://doi.org/10.1002/mrm.20945

Aso, T., Urayama, S.-I., Poupon, C., Sawamoto, N., Fukuyama, H., \& Bihan, D. L. (2009). An intrinsic diffusion response function for analyzing diffusion functional MRI time series. Neurolmage, 47(4), 1487-1495. http://doi.org/10.1016/j.neuroimage.2009.05.027

Assaf, Y., \& Basser, P. J. (2005). Composite hindered and restricted model of diffusion (CHARMED) MR imaging of the human brain. Neurolmage, 27(1), 48-58. http://doi.org/10.1016/j.neuroimage.2005.03.042

Assaf, Y., \& Cohen, Y. (1998). In vivo and in vitro bi-exponential diffusion of $\mathrm{N}$-acetyl aspartate (NAA) in rat brain: a potential structural probe? NMR in Biomedicine, 11(2), 67-74.

Assaf, Y., \& Cohen, Y. (1999). Structural information in neuronal tissue as revealed by q-space diffusion NMR spectroscopy of metabolites in bovine optic nerve. NMR in Biomedicine, 12(6), 335-344.

Assaf, Y., Blumenfeld-Katzir, T., Yovel, Y., \& Basser, P. J. (2008). Axcaliber: A method for measuring axon 
diameter distribution from diffusion MRI. Magnetic Resonance in Medicine, 59(6), 1347-1354. http://doi.org/10.1002/mrm.21577

Assaf Y, Ben-Bashat D, Chapman J, Peled S, Biton IE, Kafri M, Segev Y, Hendler T, Korczyn AD, Graif M, Cohen Y. 2002. High b-value q-space analyzed diffusion-weighted MRI: application to multiple sclerosis. Magn Reson Med. 2002 Jan;47(1):115-26.

Assaf Y, Cohen Y. 2000. Assignment of the water slow-diffusing component in the central nervous system using q-space diffusion MRS: implications for fiber tract imaging. Magn Reson Med. 2000 Feb;43(2):191-9.

Augustinack JC, Helmer K, Huber KE, Kakunoori S, Zollei L, Fischl B. Direct visualization of the perforant pathway in the human brain with ex vivo diffusion tensor imaging. Front Hum Neurosci 2010; 4:42.

Augustinack JC, Magnain C, Reuter M, van der Kouwe AJW, Boas D, Fischl B. MRI parcellation of ex vivo medial temporal lobe. Neurolmage 2014 93: 252-259.

Autio, J. A., \& Roberts, R. E. (2014). Interpreting functional diffusion tensor imaging. Frontiers in Neuroscience, 8, 68. http://doi.org/10.3389/fnins.2014.00068

Bahrami, K., Shi, F., Rekik, I., Gao, Y., \& Shen, D. (2017). 7T-guided super-resolution of 3T MRI. Medical Physics, 44(5), 1661-1677. http://doi.org/10.1002/mp.12132

Bahrami, K., Shi, F., Zong, X., Shin, H. W., An, H., \& Shen, D. (2016b). Reconstruction of 7T-Like Images From 3T MRI. IEEE Transactions on Medical Imaging, 35(9), 2085-2097.

http://doi.org/10.1109/TMI.2016.2549918

Bahrami, K., Shi, F., Rekik, I., Shen, D. (2016) Convolutional neural network for re- construction of 7T-like images from 3T MRI using appearance and anatomical features. In: MICCAI DLDLM workshop, Springer (2016) 39-47

Bai, R., Cloninger, A., Czaja, W., \& Basser, P. J. (2015). Efficient 2D MRI relaxometry using compressed sensing. Journal of Magnetic Resonance, 255, 88-99. http://doi.org/10.1016/j.jmr.2015.04.002

Bammer, R., Markl, M., Barnett, A., Acar, B., Alley, M. T., Pelc, N. J., et al. (2003). Analysis and generalized correction of the effect of spatial gradient field distortions in diffusion-weighted imaging. Magnetic Resonance in Medicine, 50(3), 560-569. http://doi.org/10.1002/mrm.10545

Barazany, D., Basser, P. J., \& Assaf, Y. (2009). In vivo measurement of axon diameter distribution in the corpus callosum of rat brain. Brain, 132(5), 1210-1220. http://doi.org/10.1093/brain/awp042

Barazany D, Jones DK, Assaf Y. 2011. AxCaliber 3D. In “Proc. ISMRM 19th Annual Meeting, Montreal, Canada".

Baron, C. A., Lebel, R. M., Wilman, A. H., \& Beaulieu, C. (2012). The effect of concomitant gradient fields on diffusion tensor imaging. Magnetic Resonance in Medicine, 68(4), 1190-1201. http://doi.org/10.1002/mrm.24120

Basser, P. J., \& Jones, D. K. (2002). Diffusion-tensor MRI: theory, experimental design and data analysis - a technical review. NMR in Biomedicine, 15(7-8), 456-467. http://doi.org/10.1002/nbm.783

Basser, P. J., \& Pierpaoli, C. (1998). A simplified method to measure the diffusion tensor from seven MR images. Magnetic Resonance in Medicine, 39(6), 928-934. http://doi.org/10.1002/mrm.1910390610

Basser, P. J., Mattiello, J., \& LeBihan, D. (1994). MR diffusion tensor spectroscopy and imaging. Biophysical Journal, 66(1), 259-267. http://doi.org/10.1016/\$0006-3495(94)80775-1

Beaulieu, C. (2002). The basis of anisotropic water diffusion in the nervous system - a technical review. NMR in Biomedicine, 15(7-8), 435-455. http://doi.org/10.1002/nbm.782

Beaulieu, C., \& Allen, P. S. (1994). Determinants of anisotropic water diffusion in nerves. Magnetic Resonance in Medicine, 31(4), 394-400. http://doi.org/10.1002/mrm.1910310408

Behrens, T. E. J., Woolrich, M. W., Jenkinson, M., Johansen-Berg, H., Nunes, R. G., Clare, S., et al. (2003). Characterization and propagation of uncertainty in diffusion-weighted MR imaging. Magnetic Resonance in Medicine, 50(5), 1077-1088. http://doi.org/10.1002/mrm.10609

Benjamini, D., \& Basser, P. J. (2016). Use of marginal distributions constrained optimization (MADCO) for accelerated 2D MRI relaxometry and diffusometry. Journal of Magnetic Resonance (San Diego, Calif. : 1997), 271, 40-45. http://doi.org/10.1016/j.jmr.2016.08.004

Benjamini, D., \& Basser, P. J. (2017). Magnetic resonance microdynamic imaging reveals distinct tissue microenvironments. Neurolmage, 163, 183-196. http://doi.org/10.1016/j.neuroimage.2017.09.033

Benjamini, D., Komlosh, M. E., Holtzclaw, L. A., Nevo, U., \& Basser, P. J. (2016). White matter microstructure 
from nonparametric axon diameter distribution mapping. Neurolmage, 135, 333-344.

http://doi.org/10.1016/j.neuroimage.2016.04.052

Berns, G. S., \& Ashwell, K. W. S. (2017). Reconstruction of the Cortical Maps of the Tasmanian Tiger and Comparison to the Tasmanian Devil. PloS One, 12(1), e0168993.

http://doi.org/10.1371/journal.pone.0168993

Berns GS, Cook PF, Foxley S, Jbabdi S, Miller KL, Marino L (2015). Diffusion tensor imaging of dolphin brains reveals direct auditory pathway to temporal lobe. Proc Royal Society B , 282: 20151203.

Bernstein MA, Zhou XJ, Polzin JA, King KF, Ganin A, Pelc NJ, Glover GH. 1998. Concomitant gradient terms in phase contrast MR: analysis and correction. Magn Reson Med. Feb;39(2):300-8.

Bertero, M., Brianzi, P., \& Pike, E. R. (1999). On the recovery and resolution of exponential relaxational rates from experimental data: Laplace transform inversions in weighted spaces. Inverse Problems, 1(1), 1-15. http://doi.org/10.1088/0266-5611/1/1/003

Bilgic, B., Setsompop, K., Cohen-Adad, J., Yendiki, A., Wald, L. L., \& Adalsteinsson, E. (2012). Accelerated diffusion spectrum imaging with compressed sensing using adaptive dictionaries. Magnetic Resonance in Medicine, 68(6), 1747-1754. http://doi.org/10.1002/mrm.24505

Bodini, B., \& Ciccarelli, O. (2014). Diffusion MRI in Neurological Disorders. In Diffusion MRI (pp. 241-255). Elsevier. http://doi.org/10.1016/B978-0-12-396460-1.00011-1

Borkowski, K., Kłodowski, K., Figiel, H., \& Krzyżak, A. T. (2017). A theoretical validation of the B-matrix spatial distribution approach to diffusion tensor imaging. Magnetic Resonance Imaging, 36, 1-6. http://doi.org/10.1016/j.mri.2016.10.002

Bourland, J. D., Nyenhuis, J. A., \& Schaefer, D. J. (1999). Physiologic effects of intense MR imaging gradient fields. Neuroimaging Clinics of North America, 9(2), 363-377.

Bourne, R. M. (2015). The trouble with apparent diffusion coefficient papers. Journal of Medical Radiation Sciences, 62(2), 89-91. http://doi.org/10.1002/jmrs.110

Bourne, R. M., Panagiotaki, E., Bongers, A., Sved, P., Watson, G., \& Alexander, D. C. (2014). Information theoretic ranking of four models of diffusion attenuation in fresh and fixed prostate tissue ex vivo. Magnetic Resonance in Medicine, 72(5), 1418-1426. http://doi.org/10.1002/mrm.25032

Branzoli, F., Techawiboonwong, A., Kan, H., Webb, A., \& Ronen, I. (2013). Functional diffusion-weighted magnetic resonance spectroscopy of the human primary visual cortex at $7 \mathrm{~T}$. Magnetic Resonance in Medicine, 69(2), 303-309. http://doi.org/10.1002/mrm.24542

Budde MD, Frank JA. Examining brain microstructure using structure tensor analysis of histological sections. Neurolmage 2012; 63:1-10.

Burcaw, L. M., Fieremans, E., \& Novikov, D. S. (2015). Mesoscopic structure of neuronal tracts from timedependent diffusion. Neurolmage, 114, 18-37. http://doi.org/10.1016/j.neuroimage.2015.03.061

Callaghan, P. T., Godefroy, S., \& Ryland, B. N. (2003). Use of the second dimension in PGSE NMR studies of porous media. Magnetic Resonance Imaging, 21(3-4), 243-248.

Cao, P., \& Wu, E. X. (2017). In vivo diffusion MRS investigation of non-water molecules in biological tissues. NMR in Biomedicine, 30(3), e3481. http://doi.org/10.1002/nbm.3481

Catani, M., Dell'Acqua, F., Vergani, F., Malik, F., Hodge, H., Roy, P., et al. (2012). Short frontal lobe connections of the human brain. Cortex; a Journal Devoted to the Study of the Nervous System and Behavior, 48(2), 273-291. http://doi.org/10.1016/j.cortex.2011.12.001

Catani, M., Robertsson, N., Beyh, A., Huynh, V., de Santiago Requejo, F., Howells, H., Barrett, R.L.C., Aiello, M., Cavaliere, C., Dyrby, T.B., Krug, K., Ptito, M., D'Arceuil, H., Forkel, S.J., Dell'Acqua, F. Short parietal lobe connections of the human and monkey brain (2017) Cortex, 97, pp. 339-357

Celik, H., Bouhrara, M., Reiter, D. A., Fishbein, K. W., \& Spencer, R. G. (2013). Stabilization of the inverse Laplace transform of multiexponential decay through introduction of a second dimension. Journal of Magnetic Resonance (San Diego, Calif. : 1997), 236, 134-139.

http://doi.org/10.1016/i.jmr.2013.07.008

Chang LC, Jones DK, Pierpaoli C. 2005. RESTORE: robust estimation of tensors by outlier rejection. Magn Reson Med. 2005 May;53(5):1088-95.

Chang LC, Walker L, Pierpaoli C. 2012. Informed RESTORE: A method for robust estimation of diffusion tensor from low redundancy datasets in the presence of physiological noise artifacts. Magn Reson Med. 2012 Nov;68(5):1654-63. doi: 10.1002/mrm.24173. Epub 2012 Jan 27.

Chronik, B. A., \& Rutt, B. K. (2001). Simple linear formulation for magnetostimulation specific to MRI 
gradient coils. Magnetic Resonance in Medicine, 45(5), 916-919.

Cloninger, A., Czaja, W., Bai, R., \& Basser, P. J. (2014). Solving 2D Fredholm Integral from Incomplete Measurements Using Compressive Sensing. SIAM Journal on Imaging Sciences, 7(3), 1775-1798. http://doi.org/10.1137/130932168

Cohen, Y., \& Assaf, Y. (2002). High b-value q-space analyzed diffusion-weighted MRS and MRI in neuronal tissues - a technical review. NMR in Biomedicine, 15(7-8), 516-542. http://doi.org/10.1002/nbm.778

Collier Q, Veraart J, Jeurissen B, den Dekker AJ, Sijbers J. 2015. Iterative reweighted linear least squares for accurate, fast, and robust estimation of diffusion magnetic resonance parameters. Magn Reson Med. 2015 Jun;73(6):2174-84. doi: 10.1002/mrm.25351. Epub 2014 Jul 1.

Cowan, M.L., Bruner, B.D., Huse, N., Dwyer, J.R., Chugh, B., Nibbering, E.T., Elsaesser, T., Miller, R.J., 2005. Ultrafast memory loss and energy redistribution in the hydrogen bond network of liquid $\mathrm{H} 2 \mathrm{O}$. Nature 434, 199-202.

D'Anna, L., Mesulam, M.M., Thiebaut De Schotten, M., Dell'Acqua, F., Murphy, D., Wieneke, C., Martersteck, A., Cobia, D., Rogalski, E., Catani, M. Frontotemporal networks and behavioral symptoms in primary progressive aphasia (2016) Neurology, 86 (15), pp. 1393-1399.

D'Arceuil, H. E., Westmoreland, S., \& de Crespigny, A. J. (2007). An approach to high resolution diffusion tensor imaging in fixed primate brain. Neurolmage, 35(2), 553-565.

http://doi.org/10.1016/j.neuroimage.2006.12.028

Daducci, A., Canales-Rodríguez, E. J., Zhang, H., Dyrby, T. B., Alexander, D. C., \& Thiran, J.-P. (2015a). Accelerated Microstructure Imaging via Convex Optimization (AMICO) from diffusion MRI data. Neurolmage, 105, 32-44. http://doi.org/10.1016/j.neuroimage.2014.10.026

Daducci, A., Dal Palù, A., Lemkaddem, A., \& Thiran, J.-P. (2015b). COMMIT: Convex optimization modeling for microstructure informed tractography. IEEE Transactions on Medical Imaging, 34(1), 246-257. http://doi.org/10.1109/TMI.2014.2352414

D'Arceuil H, de Crespigny A. 2007b. The effects of brain tissue decomposition on diffusion tensor imaging and tractography. Neurolmage. May 15 2007;36(1):64-68.

Darquié, A., Poline, J. B., Poupon, C., Saint-Jalmes, H., \& Le Bihan, D. (2001). Transient decrease in water diffusion observed in human occipital cortex during visual stimulation. Proceedings of the National Academy of Sciences, 98(16), 9391-9395. http://doi.org/10.1073/pnas.151125698

Davids, M., Guérin, B., Malzacher, M., Schad, L. R., \& Wald, L. L. (2017). Predicting Magnetostimulation Thresholds in the Peripheral Nervous System using Realistic Body Models. Scientific Reports, 7(1), 5316. http://doi.org/10.1038/s41598-017-05493-9

de Graaf, R. A., Braun, K. P., \& Nicolay, K. (2001). Single-shot diffusion trace (1)H NMR spectroscopy. Magnetic Resonance in Medicine, 45(5), 741-748.

De Santis, S., Assaf, Y., Jeurissen, B., Jones, D. K., \& Roebroeck, A. (2016a). T1 relaxometry of crossing fibres in the human brain. Neurolmage, 141, 133-142. http://doi.org/10.1016/j.neuroimage.2016.07.037

De Santis, S., Barazany, D., Jones, D. K., \& Assaf, Y. (2016b). Resolving relaxometry and diffusion properties within the same voxel in the presence of crossing fibres by combining inversion recovery and diffusionweighted acquisitions. Magnetic Resonance in Medicine, 75(1), 372-380.

http://doi.org/10.1002/mrm.25644

De Santis, S., Granberg, T., Ouellette, R., Treaba, C. A., Qiuyun Fan, Herranz, E., et al. (2017). Early axonal damage in normal appearing white matter in multiple sclerosis: Novel insights from multi-shell diffusion MRI. Conference Proceedings : ... Annual International Conference of the IEEE Engineering in Medicine and Biology Society. IEEE Engineering in Medicine and Biology Society. Annual Conference, 2017, 3024-3027. http://doi.org/10.1109/EMBC.2017.8037494

De Santis, S., Jones, D. K., \& Roebroeck, A. (2016c). Including diffusion time dependence in the extra-axonal space improves in vivo estimates of axonal diameter and density in human white matter. Neurolmage, 130, 91-103. http://doi.org/10.1016/j.neuroimage.2016.01.047

Dell'Acqua, F., Bodi, I., Slater, D., Catani, M., \& Modo, M. (2013a). MR diffusion histology and microtractography reveal mesoscale features of the human cerebellum. Cerebellum (London, England), 12(6), 923-931. http://doi.org/10.1007/s12311-013-0503-x

Dell'Acqua, F., Simmons, A., Williams, S. C. R., \& Catani, M. (2013b). Can spherical deconvolution provide more information than fiber orientations? Hindrance modulated orientational anisotropy, a true-tract specific index to characterize white matter diffusion. Human Brain Mapping, 34(10), 2464-2483. 
http://doi.org/10.1002/hbm.22080

DeLuca, G. C., Ebers, G. C., \& Esiri, M. M. (2004). Axonal loss in multiple sclerosis: a pathological survey of the corticospinal and sensory tracts. Brain, 127(Pt 5), 1009-1018.

http://doi.org/10.1093/brain/awh118

Deoni, S. C. L., Rutt, B. K., Arun, T., Pierpaoli, C., \& Jones, D. K. (2008). Gleaning multicomponent T1 and T2 information from steady-state imaging data. Magnetic Resonance in Medicine, 60(6), 1372-1387. http://doi.org/10.1002/mrm.21704

Dijkhuizen, R. M., de Graaf, R. A., Tulleken, K. A., \& Nicolay, K. (1999). Changes in the diffusion of water and intracellular metabolites after excitotoxic injury and global ischemia in neonatal rat brain. Journal of Cerebral Blood Flow and Metabolism : Official Journal of the International Society of Cerebral Blood Flow and Metabolism, 19(3), 341-349. http://doi.org/10.1097/00004647-199903000-00012

Does, M. D., Beaulieu, C., Allen, P. S., \& Snyder, R. E. (1998). Multi-component T1 relaxation and magnetisation transfer in peripheral nerve. Magnetic Resonance Imaging, 16(9), 1033-1041.

Doneva, M., Börnert, P., Eggers, H., Stehning, C., Sénégas, J., \& Mertins, A. (2010). Compressed sensing reconstruction for magnetic resonance parameter mapping. Magnetic Resonance in Medicine, 64(4), 1114-1120. http://doi.org/10.1002/mrm.22483

Drobnjak, I., \& Alexander, D. C. (2011). Optimising time-varying gradient orientation for microstructure sensitivity in diffusion-weighted MR. Journal of Magnetic Resonance (San Diego, Calif. : 1997), 212(2), 344-354. http://doi.org/10.1016/j.jmr.2011.07.017

Drobnjak, I., Siow, B., \& Alexander, D. C. (2010). Optimizing gradient waveforms for microstructure sensitivity in diffusion-weighted MR. Journal of Magnetic Resonance (San Diego, Calif. : 1997), 206(1), 41-51. http://doi.org/10.1016/j.jmr.2010.05.017

Drobnjak, I., Zhang, H., Hall, M. G., \& Alexander, D. C. (2011). The matrix formalism for generalised gradients with time-varying orientation in diffusion NMR. Journal of Magnetic Resonance (San Diego, Calif. : 1997), 210(1), 151-157. http://doi.org/10.1016/j.jmr.2011.02.022

Drobnjak, I., Zhang, H., lanuş, A., Kaden, E., \& Alexander, D. C. (2016). PGSE, OGSE, and sensitivity to axon diameter in diffusion MRI: Insight from a simulation study. Magnetic Resonance in Medicine, 75(2), 688-700. http://doi.org/10.1002/mrm.25631

Du, F., Cooper, A. J., Thida, T., Shinn, A. K., Cohen, B. M., \& Ongür, D. (2013). Myelin and axon abnormalities in schizophrenia measured with magnetic resonance imaging techniques. Biological Psychiatry, 74(6), 451-457. http://doi.org/10.1016/j.biopsych.2013.03.003

Du, Y. P., Joe Zhou, X., \& Bernstein, M. A. (2002). Correction of concomitant magnetic field-induced image artifacts in nonaxial echo-planar imaging. Magnetic Resonance in Medicine, 48(3), 509-515. http://doi.org/10.1002/mrm.10249

Duval, T., Le Vy, S., Stikov, N., Campbell, J., Mezer, A., Witzel, T., et al. (2017). g-Ratio weighted imaging of the human spinal cord in vivo. Neurolmage, 145(Pt A), 11-23. http://doi.org/10.1016/j.neuroimage.2016.09.018

Duyn, J. (2013). MR susceptibility imaging. Journal of Magnetic Resonance (San Diego, Calif. : 1997), 229, 198-207. http://doi.org/10.1016/j.jmr.2012.11.013

Duyn, J. H. (2017). Studying brain microstructure with magnetic susceptibility contrast at high-field. Neurolmage. http://doi.org/10.1016/j.neuroimage.2017.02.046

Duyn, J. H., \& Schenck, J. (2017). Contributions to magnetic susceptibility of brain tissue. NMR in Biomedicine, 30(4), e3546. http://doi.org/10.1002/nbm.3546

Dyrby, T. B., Baaré, W. F. C., Alexander, D. C., Jelsing, J., Garde, E., \& Søgaard, L. V. (2011). An ex vivo imaging pipeline for producing high-quality and high-resolution diffusion-weighted imaging datasets. Human Brain Mapping, 32(4), 544-563. http://doi.org/10.1002/hbm.21043

Dyrby, T. B., Lundell, H., Burke, M. W., Reislev, N. L., Paulson, O. B., Ptito, M., \& Siebner, H. R. (2014). Interpolation of diffusion weighted imaging datasets. Neurolmage, 103, 202-213. http://doi.org/10.1016/j.neuroimage.2014.09.005

Dyrby, T. B., S gaard, L. V., Hall, M. G., Ptito, M., \& Alexander, D. C. (2012). Contrast and stability of the axon diameter index from microstructure imaging with diffusion MRI. Magnetic Resonance in Medicine, 70(3), 711-721. http://doi.org/10.1002/mrm.24501

Ecker, C., Andrews, D., Dell'Acqua, F., Daly, E., Murphy, C., Catani, M., et al. (2016). Relationship Between Cortical Gyrification, White Matter Connectivity, and Autism Spectrum Disorder. Cerebral Cortex (New 
York, N.Y. : 1991), 26(7), 3297-3309. http://doi.org/10.1093/cercor/bhw098

Edén, M. (2003). Computer simulations in solid-state NMR. III. Powder averaging. Concepts in Magnetic Resonance Part A, 18A(1), 24-55. http://doi.org/10.1002/cmr.a.10065

Ellegood, J., Hanstock, C. C., \& Beaulieu, C. (2006). Diffusion tensor spectroscopy (DTS) of human brain. Magnetic Resonance in Medicine, 55(1), 1-8. http://doi.org/10.1002/mrm.20777

Ellegood, J., Hanstock, C. C., \& Beaulieu, C. (2011). Considerations for measuring the fractional anisotropy of metabolites with diffusion tensor spectroscopy. NMR in Biomedicine, 24(3), 270-280.

http://doi.org/10.1002/nbm.1586

Ellegood, J., McKay, R. T., Hanstock, C. C., \& Beaulieu, C. (2007). Anisotropic diffusion of metabolites in peripheral nerve using diffusion weighted magnetic resonance spectroscopy at ultra-high field. Journal of Magnetic Resonance, 184(1), 20-28. http://doi.org/10.1016/i.jmr.2006.09.008

Englund E, Sjobeck M, Brockstedt S, Latt J, Larsson EM. Diffusion tensor MRI post mortem demonstrated cerebral white matter pathology. J Neurol. 2004; 251: 350-352.

Ercan, E., Magro-Checa, C., Valabregue, R., Branzoli, F., Wood, E. T., Steup-Beekman, G. M., et al. (2016). Glial and axonal changes in systemic lupus erythematosus measured with diffusion of intracellular metabolites. Brain, 139(Pt 5), 1447-1457. http://doi.org/10.1093/brain/aww031

Eriksson, S., Lasič, S., \& Topgaard, D. (2013). Isotropic diffusion weighting in PGSE NMR by magic-angle spinning of the q-vector. Journal of Magnetic Resonance, 226, 13-18.

http://doi.org/10.1016/j.jmr.2012.10.015

Fan, Q., Witzel, T., Nummenmaa, A., Van Dijk, K. R. A., Van Horn, J. D., Drews, M. K., et al. (2016). MGH-USC Human Connectome Project datasets with ultra-high b-value diffusion MRI. Neurolmage, 124(Pt B), 1108-1114. http://doi.org/10.1016/i.neuroimage.2015.08.075

Fan Q, Nummenmaa A, Polimeni JR, Witzel T, Huang SY, Wedeen VJ, Rosen BR, Wald LL. 2017 HIgh b-value and high Resolution Integrated Diffusion (HIBRID) imaging.Neuroimage. 2017 Apr 15;150:162-176. doi: 10.1016/j.neuroimage.2017.02.002. Epub 2017 Feb 7.

Fan Q, Huang SY, Nummenmaa A, Witzel T, and Wald LL T2 relaxation rates of the fast and slow biexponential diffusion components in the in vivo corpus callosum

In International Society for Magnetic Resonance in Medicine, 25th Scientific Meeting, Honololu, Hawai'i. p. 1757838.

Ferizi, U., Schneider, T., Panagiotaki, E., Nedjati-Gilani, G., Zhang, H., Wheeler Kingshott, C. A. M., \& Alexander, D. C. (2014). A ranking of diffusion MRI compartment models with in vivo human brain data. Magnetic Resonance in Medicine, 72(6), 1785-1792. http://doi.org/10.1002/mrm.25080

Fick, R. H. J., Wassermann, D., Caruyer, E., \& Deriche, R. (2016). MAPL: Tissue microstructure estimation using Laplacian-regularized MAP-MRI and its application to HCP data. Neurolmage, 134, 365-385. http://doi.org/10.1016/j.neuroimage.2016.03.046

Fieremans, E., Jensen, J. H., \& Helpern, J. A. (2011). White matter characterization with diffusional kurtosis imaging. Neurolmage, 58(1), 177-188. http://doi.org/10.1016/j.neuroimage.2011.06.006

Fillard, P., Pennec, X., Arsigny, V., \& Ayache, N. (2007). Clinical DT-MRI estimation, smoothing, and fiber tracking with log-Euclidean metrics. IEEE Transactions on Medical Imaging, 26(11), 1472-1482. http://doi.org/10.1109/TMI.2007.899173

Fischer, H. W., Rinck, P. A., Van Haverbeke, Y., \& Muller, R. N. (1990). Nuclear relaxation of human brain gray and white matter: analysis of field dependence and implications for MRI. Magnetic Resonance in Medicine, 16(2), 317-334.

Fischer, H. W., Van Haverbeke, Y., Schmitz-Feuerhake, I., \& Muller, R. N. (1989). The uncommon longitudinal relaxation dispersion of human brain white matter. Magnetic Resonance in Medicine, 9(3), 441-446. http://doi.org/10.1002/mrm.1910090319

Foxley, S., Jbabdi, S., Clare, S., Lam, W., Ansorge, O., Douaud, G., \& Miller, K. (2014). Improving diffusionweighted imaging of post-mortem human brains: SSFP at 7 T. Neurolmage, 102 Pt 2, 579-589. http://doi.org/10.1016/i.neuroimage.2014.08.014

Foxley, S., et al. Validating tractography of high resolution post-mortem human brain at 7T with polarized light imaging. in ISMRM (Singapore, 2016).

Fritz, F., et al. High resolution diffusion MRI and tractography of post mortem human brains using kTdSTEAM at 9.4T in Org Human Brain Mapping 1567 (Vancouver, 2017)

Frost, R., Jezzard, P., Douaud, G., Clare, S., Porter, D. A., \& Miller, K. L. (2014). Scan time reduction for 
readout-segmented EPI using simultaneous multislice acceleration: Diffusion-weighted imaging at 3 and 7 Tesla. Magnetic Resonance in Medicine, 74(1), 136-149. http://doi.org/10.1002/mrm.25391

Ghosh, A., \& Deriche, R. (2016). A survey of current trends in diffusion MRI for structural brain connectivity. Journal of Neural Engineering, 13(1), 011001. http://doi.org/10.1088/1741-2560/13/1/011001

Girard, G., Daducci, A., Petit, L., Thiran, J.-P., Whittingstall, K., Deriche, R., et al. (2017). AxTract: Toward microstructure informed tractography. Human Brain Mapping, 598(11), 143-5500. http://doi.org/10.1002/hbm.23741

Glasser, M. F., Sotiropoulos, S. N., Wilson, J. A., Coalson, T. S., Fischl, B., Andersson, J. L., et al. (2013). The minimal preprocessing pipelines for the Human Connectome Project. Neurolmage, 80, 105-124. http://doi.org/10.1016/j.neuroimage.2013.04.127

Goldman, A. L., Pezawas, L., Mattay, V. S., Fischl, B., Verchinski, B. A., Chen, Q., et al. (2009). Widespread Reductions of Cortical Thickness in Schizophrenia and Spectrum Disorders and Evidence of Heritability. Archives of General Psychiatry, 66(5), 467-21. http://doi.org/10.1001/archgenpsychiatry.2009.24

Golkov, V., Dosovitskiy, A., Sperl, J. I., Menzel, M. I., Czisch, M., Samann, P., et al. (2016). q-Space Deep Learning: Twelve-Fold Shorter and Model-Free Diffusion MRI Scans. IEEE Transactions on Medical Imaging, 35(5), 1344-1351. http://doi.org/10.1109/TMI.2016.2551324

Gore, J. C., Xu, J., Colvin, D. C., Yankeelov, T. E., Parsons, E. C., \& Does, M. D. (2010). Characterization of tissue structure at varying length scales using temporal diffusion spectroscopy. NMR in Biomedicine, 23(7), 745-756. http://doi.org/10.1002/nbm.1531

Harada, M., Uno, M., Hong, F., Hisaoka, S., Nishitani, H., \& Matsuda, T. (2002). Diffusion-weighted in vivo localized proton MR spectroscopy of human cerebral ischemia and tumor. NMR in Biomedicine, 15(1), 69-74.

Hebrank FX, Gebhardt M, Siemens Medical Systems, Erlangen, Germany. 2000. SAFE-Model - A New Method for Predicting Peripheral Nerve Stimulations in MRI,In "Proc. ISMRM 8th Annual Meeting, Denver" p. 2007.

Heidemann, R. M., Anwander, A., Feiweier, T., Knösche, T. R., \& Turner, R. (2012). k-space and q-space: Combining ultra-high spatial and angular resolution in diffusion imaging using ZOOPPA at 7T. Neurolmage, 60(2), 967-978. http://doi.org/10.1016/j.neuroimage.2011.12.081

Henkelman, R. M., Stanisz, G. J., \& Graham, S. J. (2001). Magnetization transfer in MRI: a review. NMR in Biomedicine, 14(2), 57-64.

Hidalgo-Tobon, S. S., Bencsik, M., \& Bowtell, R. (2011). Reducing peripheral nerve stimulation due to gradient switching using an additional uniform field coil. Magnetic Resonance in Medicine, 66(5), 14981509. http://doi.org/10.1002/mrm.22926

Horowitz, A., Barazany, D., Tavor, I., Bernstein, M., Yovel, G., \& Assaf, Y. (2014). In vivo correlation between axon diameter and conduction velocity in the human brain. Brain Structure \& Function, 220(3), 17771788. http://doi.org/10.1007/s00429-014-0871-0

Horowitz, A., Barazany, D., Tavor, I., Yovel, G., \& Assaf, Y. (2015). Response to the comments on the paper by Horowitz et al. (2014). Brain Structure \& Function, 220(3), 1791-1792. http://doi.org/10.1007/s00429-015-1031-x

Huang, S. Y., Nummenmaa, A., Witzel, T., Duval, T., Cohen-Adad, J., Wald, L. L., \& McNab, J. A. (2015). The impact of gradient strength on in vivo diffusion MRI estimates of axon diameter. Neurolmage, 106, 464-472. http://doi.org/10.1016/j.neuroimage.2014.12.008

Huang, S. Y., Tobyne, S. M., Nummenmaa, A., Witzel, T., Wald, L. L., McNab, J. A., \& Klawiter, E. C. (2016). Characterization of Axonal Disease in Patients with Multiple Sclerosis Using High-Gradient-Diffusion MR Imaging. Radiology, 280(1), 244-251. http://doi.org/10.1148/radiol.2016151582

Hui, E. S., \& Jensen, J. H. (2015). Double-pulsed diffusional kurtosis imaging for the in vivo assessment of human brain microstructure. Neurolmage, 120, 371-381.

http://doi.org/10.1016/j.neuroimage.2015.07.013

Hursh JB (1939) Conduction velocity and diameter of nerve fibers. Am J Physiol 127:131-139.

Hutsler, J. J., \& Zhang, H. (2010). Increased dendritic spine densities on cortical projection neurons in autism spectrum disorders. Brain Research, 1309, 83-94.

http://doi.org/10.1016/j.brainres.2009.09.120

Hüppi, P. (2010). Diffusion Tensor Imaging in Brain Development. In Diffusion MRI (pp. 500-517). Oxford University Press. http://doi.org/10.1093/med/9780195369779.003.0030 
lima, M., \& Le Bihan, D. (2016). Clinical Intravoxel Incoherent Motion and Diffusion MR Imaging: Past, Present, and Future. Radiology, 278(1), 13-32. http://doi.org/10.1148/radiol.2015150244

Innocenti, G. M., Caminiti, R., \& Aboitiz, F. (2015). Comments on the paper by Horowitz et al. (2014). Brain Structure \& Function, 220(3), 1789-1790. http://doi.org/10.1007/s00429-014-0974-7

Istratov, A. A., \& Vyvenko, O. F. (1999). Exponential analysis in physical phenomena. Review of Scientific Instruments, 70(2), 1233-1257. http://doi.org/10.1063/1.1149581

Reilly JP. (1995). Nerve Stimulation of Cows and Other Farm Animals by Time-varying Magnetic Fields. Transactions of the ASAE, 38(5), 1487-1494. http://doi.org/10.13031/2013.27974

Jbabdi, S., Woolrich, M. W., Andersson, J. L. R., \& Behrens, T. E. J. (2007). A Bayesian framework for global tractography. Neurolmage, 37(1), 116-129. http://doi.org/10.1016/j.neuroimage.2007.04.039

Jbabdi S, Lehman JF, Haber SN, Behrens TE. Human and monkey ventral prefrontal fibers use the same organizational principles to reach their targets: tracing versus tractography. $J$ Neurosci. Feb 13 2013;33(7):3190-3201.

Jelescu, I. O., Veraart, J., Fieremans, E., \& Novikov, D. S. (2016). Degeneracy in model parameter estimation for multi-compartmental diffusion in neuronal tissue. NMR in Biomedicine, 29(1), 33-47. http://doi.org/10.1002/nbm.3450

Jensen, J. H. (2015). Stejskal's formula for multiple-pulsed diffusion MRI. Magnetic Resonance Imaging, 33(9), 1182-1186. http://doi.org/10.1016/j.mri.2015.07.001

Jespersen, S. N., Lundell, H., Sønderby, C. K., \& Dyrby, T. B. (2013). Orientationally invariant metrics of apparent compartment eccentricity from double pulsed field gradient diffusion experiments. NMR in Biomedicine, 26(12), 1647-1662. http://doi.org/10.1002/nbm.2999

Jeurissen B, Tournier JD, Dhollander T, Connelly A, Sijbers J. 2014. Multi-tissue constrained spherical deconvolution for improved analysis of multi-shell diffusion MRI data. Neuroimage. 2014 Dec;103:411-26. doi: 10.1016/j.neuroimage.2014.07.061. Epub 2014 Aug 7.

Jiang X, Li H, Xie J, McKinley ET, Zhao P, Gore JC, Xu J. 2017. In vivo imaging of cancer cell size and cellularity using temporal diffusion spectroscopy. Magn Reson Med. 2017 Jul;78(1):156-164. doi: 10.1002/mrm.26356. Epub 2016 Aug 6

Johansen-Berg, H. (2017). Imaging and stimulating adaptive brain plasticity. European Neuropsychopharmacology, 27, S1. http://doi.org/10.1016/S0924-977X(17)30067-6

Jones, D. K., Knösche, T. R., \& Turner, R. (2013). White matter integrity, fiber count, and other fallacies: the do"s and don"ts of diffusion MRI. Neurolmage, 73, 239-254.

http://doi.org/10.1016/j.neuroimage.2012.06.081

Jovicich, J., Czanner, S., Greve, D., Haley, E., van der Kouwe, A., Gollub, R., et al. (2006). Reliability in multisite structural MRI studies: effects of gradient non-linearity correction on phantom and human data. Neurolmage, 30(2), 436-443. http://doi.org/10.1016/i.neuroimage.2005.09.046

Jungwirth, P., 2011. Physical chemistry: Water's wafer-thin surface. Nature 474, 168-169.

Kaden, E., Kruggel, F., \& Alexander, D. C. (2016). Quantitative mapping of the per-axon diffusion coefficients in brain white matter. Magnetic Resonance in Medicine, 75(4), 1752-1763. http://doi.org/10.1002/mrm.25734

Kaden E, Kruggel F, Alexander DC. 2016a Quantitative mapping of the per-axon diffusion coefficients in brain white matter. Magn Reson Med. 75(4):1752-63. doi: 10.1002/mrm.25734. Epub 2015 May 13.

Kaden E, Kelm ND, Carson RP, Does MD, Alexander DC. 2016b. Multi-compartment microscopic diffusion imaging. Neuroimage. 139:346-359.

Kaden E, Rudrapatna SU, Barskaya IY,Does MD, Jones DK, Alexander DC. 2018. Microscopic susceptibility anisotropy imaging: A clinically viable gradient-echo MRI technique. In Proceedings of ISMRM Annual Scientific Meeting, Paris, France. 2018.

Kakkad, S., Zhang, J., Akhbardeh, A., Jacob, D., Krishnamachary, B., Solaiyappan, M., et al. (2016). Collagen fibers mediate MRI-detected water diffusion and anisotropy in breast cancers. Neoplasia (New York, N.Y.), 18(10), 585-593. http://doi.org/10.1016/j.neo.2016.08.004

Kakkar, L. S., Bennett, O. F., Siow, B., Richardson, S., lanuş, A., Quick, T., et al. (2017). Low frequency oscillating gradient spin-echo sequences improve sensitivity to axon diameter: An experimental study in viable nerve tissue. Neurolmage. http://doi.org/10.1016/j.neuroimage.2017.07.060

Kamagata, K., Zalesky, A., Hatano, T., Ueda, R., Di Biase, M. A., Okuzumi, A., et al. (2017). Gray Matter Abnormalities in Idiopathic Parkinson's Disease: Evaluation by Diffusional Kurtosis Imaging and Neurite 
Orientation Dispersion and Density Imaging. Human Brain Mapping, 45, 770.

http://doi.org/10.1002/hbm.23628

Karlicek, R. F., Jr., \& Lowe, I. J. (1980). A modified pulsed gradient technique for measuring diffusion in the presence of large background gradients. Journal of Magnetic Resonance (1969), 37(1), 75-91. http://doi.org/10.1016/0022-2364(80)90095-5

Kim, D., Doyle, E. K., Wisnowski, J. L., Kim, J. H., \& Haldar, J. P. (2017). Diffusion-relaxation correlation spectroscopic imaging: A multidimensional approach for probing microstructure. Magnetic Resonance in Medicine, 78(6), 2236-2249. http://doi.org/10.1002/mrm.26629

King MD, Houseman J, Roussel SA, van Bruggen N, Williams SR, Gadian DG. 1994. Q-Space imaging of the brain. Magn Reson Med. 1994 Dec;32(6):707-13.

Kleinnijenhuis M, Zerbi V, Kusters B, Slump CH, Barth M, van Cappellen van Walsum AM. Layer-specific diffusion weighted imaging in human primary visual cortex in vitro. Cortex 2012.

Kleinnijenhuis M, van Mourik T, Norris DG, Ruiter DJ, van Cappellen van Walsum AM, Barth M. Diffusion tensor characteristics of gyrencephaly using high resolution diffusion MRI in vivo at 7T. Neurolmage 2015.

Koay, C. G., Carew, J. D., Alexander, A. L., Basser, P. J., \& Meyerand, M. E. (2006). Investigation of anomalous estimates of tensor-derived quantities in diffusion tensor imaging. Magnetic Resonance in Medicine, 55(4), 930-936. http://doi.org/10.1002/mrm.20832

Koh, D.-M., Collins, D. J., \& Orton, M. R. (2011). Intravoxel incoherent motion in body diffusion-weighted MRI: reality and challenges. AJR. American Journal of Roentgenology, 196(6), 1351-1361. http://doi.org/10.2214/AJR.10.5515

Kolasinski J, Takahashi E, Stevens AA, Benner T, Fischl B, Zollei L, Grant, PE. Radial and tangential neuronal migration pathways in the human fetal brain: Anatomically distinct patterns of diffusion MRI coherence. Neurolmage 2013. 79: 412-422.

Kroenke, C. D., Ackerman, J. J. H., \& Yablonskiy, D. A. (2004). On the nature of the NAA diffusion attenuated MR signal in the central nervous system. Magnetic Resonance in Medicine, 52(5), 1052-1059. http://doi.org/10.1002/mrm.20260

Lai, S., Glover, G. H., \& Haacke, E. M. (2000). Spatial Selectivity of BOLD Contrast: Effects In and Around Draining Veins. In Functional MRI (pp. 221-231). Berlin, Heidelberg: Springer Berlin Heidelberg. http://doi.org/10.1007/978-3-642-58716-0_20

Lampinen, B., Szczepankiewicz, F., Mårtensson, J., van Westen, D., Sundgren, P. C., \& Nilsson, M. (2017). Neurite density imaging versus imaging of microscopic anisotropy in diffusion MRI: A model comparison using spherical tensor encoding. Neurolmage, 147, 517-531. http://doi.org/10.1016/j.neuroimage.2016.11.053

Lampinen, B., Szczepankiewicz, F., van Westen, D., Englund, E., Sundgren, P. C., Lätt. J., Ståhlberg. F., Nilsson M. 2017b. Optimal Experimental Design for Filter Exchange Imaging: Apparent Exchange Rate Measurements in the Healthy Brain and in Intracranial Tumors. Magnetic Resonance in Medicine, 77, 1104-1114.

Landman, B., Bazin, P.-L., \& Prince, J. (2007). Diffusion Tensor Estimation by Maximizing Rician Likelihood. Proceedings. IEEE International Conference on Computer Vision, 1-8.

Larkman, D. J., Hajnal, J. V., Herlihy, A. H., Coutts, G. A., Young, I. R., \& Ehnholm, G. (2001). Use of multicoil arrays for separation of signal from multiple slices simultaneously excited. Journal of Magnetic Resonance Imaging : JMRI, 13(2), 313-317.

Larkman, D.J., Nunes, R.G., 2007. Parallel magnetic resonance imaging. Phys Med Biol 52, R15-55.

Larsson EM, Englund E, Sjobeck M, Latt J, Brockstedt S. MRI with Diffusion Tensor Imaging Post-Mortem at 3.0 T in a Patient with Frontotemporal Dementia. Dementia and Geriatric Cognitive Disorders. 2004; 17:316-319

Lasič, S., Szczepankiewicz, F., Eriksson, S., Nilsson, M., \& Topgaard, D. (2014). Microanisotropy imaging: quantification of microscopic diffusion anisotropy and orientational order parameter by diffusion MRI with magic-angle spinning of the q-vector. Frontiers in Physics, 2. http://doi.org/10.3389/fphy.2014.00011

Latour, L. L., Hasegawa, Y., Formato, J. E., Fisher, M., \& Sotak, C. H. (1994). Spreading waves of decreased diffusion coefficient after cortical stimulation in the rat brain. Magnetic Resonance in Medicine, 32(2), 189-198. 
Lätt J, Nilsson M, Wirestam R, Johansson E, Larsson EM, Stahlberg F, Brockstedt S.

2008. In vivo visualization of displacement-distribution-derived parameters in q-space imaging.

Magn Reson Imaging. 2008 Jan;26(1):77-87. Epub 2007 Jun 20

Lawrenz, M., \& Finsterbusch, J. (2013). Double-wave-vector diffusion-weighted imaging reveals microscopic diffusion anisotropy in the living human brain. Magnetic Resonance in Medicine, 69(4), 1072-1082. http://doi.org/10.1002/mrm.24347

Lawrenz, M., Brassen, S., \& Finsterbusch, J. (2016). Microscopic diffusion anisotropy in the human brain: Age-related changes. Neurolmage, 141, 313-325. http://doi.org/10.1016/j.neuroimage.2016.07.031

Le Bihan, D., Breton, E., Lallemand, D., Grenier, P., Cabanis, E., \& Laval-Jeantet, M. (1986). MR imaging of intravoxel incoherent motions: application to diffusion and perfusion in neurologic disorders. Radiology, 161(2), 401-407. http://doi.org/10.1148/radiology.161.2.3763909

Le Bihan D1, Poupon C, Amadon A, Lethimonnier F. 2006. Artifacts and pitfalls in diffusion MRI. J Magn Reson Imaging. Sep;24(3):478-88.

Le Bihan, D., Joly, O., Aso, T., Uhrig, L., Poupon, C., Tani, N., et al. (2012). Brain tissue water comes in two pools: Evidence from diffusion and R2' measurements with USPIOs in non human primates. Neurolmage, 62(1), 9-16. http://doi.org/10.1016/j.neuroimage.2012.05.011

Le Bihan, D., Urayama, S.-I., Aso, T., Hanakawa, T., \& Fukuyama, H. (2006). Direct and fast detection of neuronal activation in the human brain with diffusion MRI. Proceedings of the National Academy of Sciences, 103(21), 8263-8268. http://doi.org/10.1073/pnas.0600644103

Lee, S.-K., Mathieu, J.-B., Graziani, D., Piel, J., Budesheim, E., Fiveland, E., et al. (2016). Peripheral nerve stimulation characteristics of an asymmetric head-only gradient coil compatible with a high-channelcount receiver array. Magnetic Resonance in Medicine, 76(6), 1939-1950. http://doi.org/10.1002/mrm.26044

Lemberskiy, G., Rosenkrantz, A. B., Veraart, J., Taneja, S. S., Novikov, D. S., \& Fieremans, E. (2017). TimeDependent Diffusion in Prostate Cancer. Investigative Radiology, 52(7), 405-411. http://doi.org/10.1097/RLI.0000000000000356

Leuze CWU, Anwander A, Bazin PL, Dhital B, Stüber C, Reimann, K Geyer S, Turner R. Layer-Specific Intracortical Connectivity Revealed with Diffusion MRI . Cerebral Cortex 2012.

Ligneul, C., Palombo, M., \& Valette, J. (2017). Metabolite diffusion up to very high b in the mouse brain in vivo: Revisiting the potential correlation between relaxation and diffusion properties. Magnetic Resonance in Medicine, 77(4), 1390-1398. http://doi.org/10.1002/mrm.26217

Ligneul C. et al. Assessing metabolic and structural alterations of brain cells in the APP/PS1/tauP301L mouse model of Alzheimer's disease using MRS and diffusion-weighted MRS in vivo. Proc. Intl. Soc. Mag. Reson. Med. 24, 2016, \#0112;

Ligneul C. et al. Probing Alterations of Cellular Metabolism and Structure in CNTF-Induced Mouse Model of Astrocytic Activation Using in Vivo MRS and Diffusion-Weighted MRS, Proc. IntI. Soc. Mag. Reson. Med. 25, 2017, \#0410

Lim, K. O., \& Helpern, J. A. (2002). Neuropsychiatric applications of DTI - a review. NMR in Biomedicine, 15(7-8), 587-593. http://doi.org/10.1002/nbm.789

Liu, J., Gasbarra, D., \& Railavo, J. (2016). Fast estimation of diffusion tensors under Rician noise by the EM algorithm. Journal of Neuroscience Methods, 257, 147-158. http://doi.org/10.1016/j.jneumeth.2015.09.029

Logothetis, N. K., \& Wandell, B. A. (2004). Interpreting the BOLD Signal. Annual Review of Physiology, 66(1), 735-769. http://doi.org/10.1146/annurev.physiol.66.082602.092845

Louis, E. D., Lee, M., Babij, R., Ma, K., Cortés, E., Vonsattel, J.-P. G., \& Faust, P. L. (2014). Reduced Purkinje cell dendritic arborization and loss of dendritic spines in essential tremor. Brain, 137(Pt 12), 31423148. http://doi.org/10.1093/brain/awu314

Lundell $H, \underline{\text { Sønderby CK}, ~ D y r b y ~ T B . ~ D i f f u s i o n ~ w e i g h t e d ~ i m a g i n g ~ w i t h ~ c i r c u l a r l y ~ p o l a r i z e d ~ o s c i l l a t i n g ~}$ gradients. Magn Reson Med. 2015 Mar;73(3):1171-6. doi: 10.1002/mrm.25211. Epub 2014 Mar 17.

Lundell, H., Alexander, D. C., \& Dyrby, T. B. (2014). High angular resolution diffusion imaging with stimulated echoes: compensation and correction in experiment design and analysis. NMR in Biomedicine, 27(8), 918-925. http://doi.org/10.1002/nbm.3137

Lustig, M., Donoho, D. L., Santos, J. M., \& Pauly, J. M. (2008). Compressed Sensing MRI. IEEE Signal Processing Magazine, 25(2), 72-82. http://doi.org/10.1109/MSP.2007.914728 
Lustig, M., Donoho, D., \& Pauly, J. M. (2007). Sparse MRI: The application of compressed sensing for rapid MR imaging. Magnetic Resonance in Medicine, 58(6), 1182-1195. http://doi.org/10.1002/mrm.21391

Ma, D., Gulani, V., Seiberlich, N., Liu, K., Sunshine, J. L., Duerk, J. L., \& Griswold, M. A. (2013). Magnetic resonance fingerprinting. Nature, 495(7440), 187-192. http://doi.org/10.1038/nature11971

Ma, D., Wright, K., Seiberlich, N., Gulani, V., \& Griswold, M. A. (2014). Simultaneous T1, T2, diffusion and proton density quantification with MR fingerprinting. Proceedings of the 22nd ....

MacKay, A. L., \& Laule, C. (2007). Myelin Water Imaging (4 ed., Vol. 103). Chichester, UK: John Wiley \& Sons, Ltd. http://doi.org/10.1002/9780470034590.emrstm1270

MacKay, A., Whittall, K., Adler, J., Li, D., Paty, D., \& Graeb, D. (1994). In vivo visualization of myelin water in brain by magnetic resonance. Magnetic Resonance in Medicine, 31(6), 673-677.

Malyarenko, D. I., Ross, B. D., \& Chenevert, T. L. (2014). Analysis and correction of gradient nonlinearity bias in apparent diffusion coefficient measurements. Magnetic Resonance in Medicine, 71(3), 13121323. http://doi.org/10.1002/mrm.24773

Mangin, J. F., Fillard, P., Cointepas, Y., Le Bihan, D., Frouin, V., \& Poupon, C. (2013). Toward global tractography. Neurolmage, 80, 290-296. http://doi.org/10.1016/j.neuroimage.2013.04.009

Marks, B. L., Katz, L. M., Styner, M., \& Smith, J. K. (2011). Aerobic fitness and obesity: relationship to cerebral white matter integrity in the brain of active and sedentary older adults. British Journal of Sports Medicine, 45(15), 1208-1215. http://doi.org/10.1136/bjsm.2009.068114

Marzi, S., Piludu, F., \& Vidiri, A. (2013). Assessment of diffusion parameters by intravoxel incoherent motion MRI in head and neck squamous cell carcinoma. NMR in Biomedicine, 26(12), 1806-1814. http://doi.org/10.1002/nbm.3020

Mattiello, J., Basser, P. J., \& Le Bihan, D. (1997). The b matrix in diffusion tensor echo-planar imaging. Magnetic Resonance in Medicine, 37(2), 292-300.

Mattiello, J., Basser, P. J., \& LeBihan, D. (1994). Analytical Expressions for the b Matrix in NMR Diffusion Imaging and Spectroscopy. Journal of Magnetic Resonance, Series A, 108(2), 131-141. http://doi.org/10.1006/jmra.1994.1103

McNab, J. A., \& Miller, K. L. (2010). Steady-state diffusion-weighted imaging: theory, acquisition and analysis. NMR in Biomedicine, 23(7), 781-793. http://doi.org/10.1002/nbm.1509

McNab, J. A., Edlow, B. L., Witzel, T., Huang, S. Y., Bhat, H., Heberlein, K., et al. (2013). The Human Connectome Project and beyond: initial applications of 300 mT/m gradients. Neurolmage, 80, 234245. http://doi.org/10.1016/i.neuroimage.2013.05.074

Maier-Hein KH, Neher PF, Houde JC, Côté MA, Garyfallidis E, Zhong J, Chamberland M, Yeh FC, Lin YC, Ji Q, Reddick WE, Glass JO, Chen DQ, Feng Y, Gao C, Wu Y, Ma J, Renjie H, Li Q, Westin CF, DeslauriersGauthier S, González JOO, Paquette M, St-Jean S, Girard G, Rheault F, Sidhu J, Tax CMW, Guo F, Mesri HY, Dávid S, Froeling M, Heemskerk AM, Leemans A, Boré A, Pinsard B, Bedetti C, Desrosiers $M$, Brambati S, Doyon J, Sarica A, Vasta R, Cerasa A, Quattrone A, Yeatman J, Khan AR, Hodges W, Alexander S, Romascano D, Barakovic M, Auría A, Esteban O, Lemkaddem A, Thiran JP, Cetingul HE, Odry BL, Mailhe B, Nadar MS, Pizzagalli F, Prasad G, Villalon-Reina JE, Galvis J, Thompson PM, Requejo FS, Laguna PL, Lacerda LM, Barrett R, Dell'Acqua F, Catani M, Petit L, Caruyer E, Daducci A, Dyrby TB, Holland-Letz T, Hilgetag CC, Stieltjes B, Descoteaux M.2017. The challenge of mapping the human connectome based on diffusion tractography. Nat Commun. 2017 8(1):1349. doi: 10.1038/s41467-01701285-x.

Mars RB, Neubert FX, Verhagen L, Sallet J, Miller KL, Dunbar RIM, Barton RA (2014). Primate comparative neuroscience using magnetic resonance imaging: Promises and challenges. Frontiers in Neuroscience, 8: 298.

McNab JA, Voets NL, Jenkinson N, Squier W, Miller KL, Goodwin GM, Aziz T (2009). Reduced limbic connections may contraindicate subgenual cingulate deep brain stimulation for intractable depression. Journal of Neurosurgery, 111: 790-784.

Meier, C., Zwanger, M., Feiweier, T., \& Porter, D. (2008). Concomitant field terms for asymmetric gradient coils: Consequences for diffusion, flow, and echo-planar imaging. Magnetic Resonance in Medicine, 60(1), 128-134. http://doi.org/10.1002/mrm.21615

Merboldt, K. D., Hänicke, W., \& Frahm, J. (1991). Diffusion imaging using stimulated echoes. Magnetic Resonance in Medicine, 19(2), 233-239.

Miller, K. L., Bulte, D. P., Devlin, H., Robson, M. D., Wise, R. G., Woolrich, M. W., et al. (2007). Evidence for a 
vascular contribution to diffusion FMRI at high $b$ value. Proceedings of the National Academy of Sciences of the United States of America, 104(52), 20967-20972.

http://doi.org/10.1073/pnas.0707257105

Miller, K. L., McNab, J. A., Jbabdi, S., \& Douaud, G. (2012). Diffusion tractography of post-mortem human brains: optimization and comparison of spin echo and steady-state free precession techniques. Neurolmage, 59(3), 2284-2297. http://doi.org/10.1016/j.neuroimage.2011.09.054

Miller, K. L., Stagg, C. J., Douaud, G., Jbabdi, S., Smith, S. M., Behrens, T. E. J., et al. (2011). Diffusion imaging of whole, post-mortem human brains on a clinical MRI scanner. Neurolmage, 57(1), 167-181. http://doi.org/10.1016/j.neuroimage.2011.03.070

Minati, L., Grisoli, M., \& Bruzzone, M. G. (2007). MR spectroscopy, functional MRI, and diffusion-tensor imaging in the aging brain: a conceptual review. Journal of Geriatric Psychiatry and Neurology, 20(1), 3-21. http://doi.org/10.1177/0891988706297089

Mirzaalian H, de Pierrefeu A, Savadjiev P, Pasternak O, Bouix S, Kubicki M, Westin CF, Shenton ME, Rathi Y. 2015. Harmonizing Diffusion MRI Data Across Multiple Sites and Scanners.Med Image Comput Comput Assist Interv. 2015 Oct;9349:12-19. Epub 2015 Nov 18.

Mitra, P. (1995). Multiple wave-vector extensions of the NMR pulsed-field-gradient spin-echo diffusion measurement. Physical Review. B, Condensed Matter, 51(21), 15074-15078.

Mlynárik, V., Gruber, S., \& Moser, E. (2001). Proton T (1) and T (2) relaxation times of human brain metabolites at 3 Tesla. NMR in Biomedicine, 14(5), 325-331.

Modo M, Hitchens TK, Liu JR, Richardson RM. Detection of Aberrant Hippocampal Mossy Fiber Connections: Ex Vivo Mesoscale Diffusion MRI and Microtractography With Histological Validation in a Patient With Uncontrolled Temporal Lobe Epilepsy. Hum Brain Mapp. Feb 2016;37(2):780-795.

Moeller, S., Yacoub, E., Olman, C. A., Auerbach, E., Strupp, J., Harel, N., \& Ugurbil, K. (2010). Multiband multislice GE-EPI at 7 tesla, with 16 -fold acceleration using partial parallel imaging with application to high spatial and temporal whole-brain fMRI. Magnetic Resonance in Medicine, 63(5), 1144-1153. http://doi.org/10.1002/mrm.22361

Mohammadi, S., Nagy, Z., Möller, H. E., Symms, M. R., Carmichael, D. W., Josephs, O., \& Weiskopf, N. (2012). The effect of local perturbation fields on human DTI: characterisation, measurement and correction. Neurolmage, 60(1), 562-570. http://doi.org/10.1016/j.neuroimage.2011.12.009

Mollink, J., Kleinnijenhuis, M., Cappellen van Walsum, A.-M. V., Sotiropoulos, S. N., Cottaar, M., Mirfin, C., et al. (2017). Evaluating fibre orientation dispersion in white matter: Comparison of diffusion MRI, histology and polarized light imaging. Neurolmage, 157, 561-574. http://doi.org/10.1016/j.neuroimage.2017.06.001

Mollink J, van Baarsen K, Dederen PJWC, Foxley S, Miller KL, Slump C, Grotenhuis JA, Kleinnijenhuis M, van Cappellen van Walsum AM (2016). Dentatorubrothalamic tract localization with post mortem MR diffusion tractography compared to histological 3D reconstruction. Brain Structure and Function , 221: 3487-3501.

Moonen, C. T., van Zijl, P. C., Le Bihan, D., \& DesPres, D. (1990). In vivo NMR diffusion spectroscopy: 31P application to phosphorus metabolites in muscle. Magnetic Resonance in Medicine, 13(3), 467-477.

Mori, S., \& van Zijl, P. C. M. (1995). Diffusion Weighting by the Trace of the Diffusion Tensor within a Single Scan. Magnetic Resonance in Medicine, 33(1), 41-52. http://doi.org/10.1002/mrm.1910330107

Murphy, K., Bodurka, J., Bandettini, P.A., 2007. How long to scan? The relationship between fMRI temporal signal to noise ratio and necessary scan duration. Neuroimage 34, 565-574.

Nair, G., Tanahashi, Y., Low, H. P., Billings-Gagliardi, S., Schwartz, W. J., \& Duong, T. Q. (2005). Myelination and long diffusion times alter diffusion-tensor-imaging contrast in myelin-deficient shiverer mice. Neurolmage, 28(1), 165-174. http://doi.org/10.1016/j.neuroimage.2005.05.049

Najac, C., Branzoli, F., Ronen, I., \& Valette, J. (2016). Brain intracellular metabolites are freely diffusing along cell fibers in grey and white matter, as measured by diffusion-weighted MR spectroscopy in the human brain at 7 T. Brain Structure \& Function, 221(3), 1245-1254. http://doi.org/10.1007/s00429014-0968-5

Nedjati-Gilani GL, Schneider T, Hall MG, Cawley N, Hill I, Ciccarelli O, Drobnjak I, Wheeler-Kingshott CAMG, Alexander DC. (2017) Machine learning based compartment models with permeability for white matter microstructure imaging. Neuroimage. Apr 15;150:119-135. doi: 10.1016/j.neuroimage.2017.02.013. Epub 2017 Feb 8. 
Nicolay, K., Braun, K. P., Graaf, R. A., Dijkhuizen, R. M., \& Kruiskamp, M. J. (2001). Diffusion NMR spectroscopy. NMR in Biomedicine, 14(2), 94-111.

Nilsson, M., Lasič, S., Drobnjak, I., Topgaard, D., \& Westin, C. F. (2017). Resolution limit of cylinder diameter estimation by diffusion MRI: The impact of gradient waveform and orientation dispersion. NMR in Biomedicine, 30(7), e3711. http://doi.org/10.1002/nbm.3711

Nilsson, M., Lätt, J., Ståhlberg, F., van Westen, D., \& Hagslätt, H. (2012). The importance of axonal undulation in diffusion MR measurements: a Monte Carlo simulation study. NMR in Biomedicine, 25(5), 795-805. http://doi.org/10.1002/nbm.1795

Ning, L., Setsompop, K., Michailovich, O., Makris, N., Westin, C. F., \& Rathi, Y. (2015). A Compressed-Sensing Approach for Super-Resolution Reconstruction of Diffusion MRI. In Medical Image Computing and Computer-Assisted Intervention -- MICCAI 2015 (Vol. 9123, pp. 57-68). Cham: Springer International Publishing. http://doi.org/10.1007/978-3-319-19992-4_5

Norris, D. G., \& Hutchison, J. M. S. (1990). Concomitant magnetic field gradients and their effects on imaging at low magnetic field strengths. Magnetic Resonance Imaging, 8(1), 33-37. http://doi.org/10.1016/0730-725X(90)90209-K

Novikov DS, Veraart J, Jelescu IO, Fieremans E. 2018 Rotationally-invariant mapping of scalar and orientational metrics of neuronal microstructure with diffusion MRI. Neuroimage. 2018 Mar 12. pii: S1053-8119(18)30191-5. doi: 10.1016/j.neuroimage.2018.03.006. [Epub ahead of print]

Novikov DS, Kiselev VG, Jespersen SN. 2018. On modeling. Magn Reson Med. 2018 Jun;79(6):3172-3193. doi: 10.1002/mrm.27101. Epub 2018 Mar 1.

Novikov, Dmitry S., , Jespersen SN, Valerii G. Kiselev VG, Fieremans E. Quantifying brain microstructure with diffusion MRI: Theory and parameter estimation arXiv preprint arXiv:1612.02059 (2016)

Novikov DS, Veraart J, Jelescu IO, Fieremans E. 2017. Mapping orientational and microstructural metrics of neuronal integrity with in vivo diffusion MRI arXiv:1609.09144

O'Connor, J. P. B., Aboagye, E. O., Adams, J. E., Aerts, H. J. W. L., Barrington, S. F., Beer, A. J., et al. (2017). Imaging biomarker roadmap for cancer studies. (Vol. 14, pp. 169-186). Presented at the Nature reviews. Clinical oncology, Nature Publishing Group. http://doi.org/10.1038/nrclinonc.2016.162

O'Connor, K. M., Barest, G., Moritani, T., Sakai, O., \& Mian, A. (2013). "Dazed and diffused": making sense of diffusion abnormalities in neurologic pathologies. The British Journal of Radiology, 86(1032), 20130599. http://doi.org/10.1259/bjr.20130599

Olivares R, Montiel J, Aboitiz F. (2001) Species differences and similarities in the fine structure of the mammalian corpus callosum. Brain Behav Evol.57(2):98-105.

Olman, C. A., Inati, S., \& Heeger, D. J. (2007). The effect of large veins on spatial localization with GE BOLD at $3 \mathrm{~T}$ : Displacement, not blurring. Neurolmage, 34(3), 1126-1135. http://doi.org/10.1016/j.neuroimage.2006.08.045

Ong, H. H., \& Wehrli, F. W. (2010). Quantifying axon diameter and intra-cellular volume fraction in excised mouse spinal cord with q-space imaging. Neurolmage, 51(4), 1360-1366. http://doi.org/10.1016/j.neuroimage.2010.03.063

Ong, H. H., Wright, A. C., Wehrli, S. L., Souza, A., Schwartz, E. D., Hwang, S. N., \& Wehrli, F. W. (2008). Indirect measurement of regional axon diameter in excised mouse spinal cord with q-space imaging: simulation and experimental studies. Neurolmage, 40(4), 1619-1632. http://doi.org/10.1016/j.neuroimage.2008.01.017

Özarslan E, Koay CG, Shepherd TM, Komlosh ME, İrfanoğlu MO, Pierpaoli C, Basser PJ. 2013

Mean apparent propagator (MAP) MRI: a novel diffusion imaging method for mapping tissue microstructure. Neuroimage. 2013 Sep;78:16-32. doi: 10.1016/j.neuroimage.2013.04.016. Epub 2013 Apr 13.

Palombo, M., Ligneul, C., \& Valette, J. (2016a). Modeling diffusion of intracellular metabolites in the mouse brain up to very high diffusion-weighting: Diffusion in long fibers (almost) accounts for nonmonoexponential attenuation. Magnetic Resonance in Medicine, 77(1), 343-350. http://doi.org/10.1002/mrm.26548

Palombo, M., Ligneul, C., Hernandez-Garzon, E., \& Valette, J. (2017a). Can we detect the effect of spines and leaflets on the diffusion of brain intracellular metabolites? Neurolmage. http://doi.org/10.1016/j.neuroimage.2017.05.003

Palombo, M., Ligneul, C., Najac, C., Le Douce, J., Flament, J., Escartin, C., et al. (2016b). New paradigm to 
assess brain cell morphology by diffusion-weighted MR spectroscopy in vivo. Proceedings of the National Academy of Sciences of the United States of America, 113(24), 6671-6676. http://doi.org/10.1073/pnas.1504327113

Palombo, M., Shemesh, N., Ronen, I., \& Valette, J. (2017b). Insights into brain microstructure from in vivo DW-MRS. Neurolmage. http://doi.org/10.1016/j.neuroimage.2017.11.028

Pampel, A., Jochimsen, T. H., \& Möller, H. E. (2010). BOLD background gradient contributions in diffusionweighted fMRI--comparison of spin-echo and twice-refocused spin-echo sequences. NMR in Biomedicine, 23(6), 610-618. http://doi.org/10.1002/nbm.1502

Panagiotaki, Eleftheria, Chan, R. W., Dikaios, N., Ahmed, H. U., O'Callaghan, J., Freeman, A., et al. (2015). Microstructural characterization of normal and malignant human prostate tissue with vascular, extracellular, and restricted diffusion for cytometry in tumours magnetic resonance imaging. Investigative Radiology, 50(4), 218-227. http://doi.org/10.1097/RLI.0000000000000115

Panagiotaki, Eleftheria, Schneider, T., Siow, B., Hall, M. G., Lythgoe, M. F., \& Alexander, D. C. (2012). Compartment models of the diffusion MR signal in brain white matter: a taxonomy and comparison. Neurolmage, 59(3), 2241-2254. http://doi.org/10.1016/j.neuroimage.2011.09.081

Panagiotaki, Eletheria, Walker-Samuel, S., Siow, B., Johnson, S. P., Rajkumar, V., Pedley, R. B., et al. (2014). Noninvasive quantification of solid tumor microstructure using VERDICT MRI. Cancer Research, 74(7), 1902-1912. http://doi.org/10.1158/0008-5472.CAN-13-2511

Parker, Greg D.; Marshall, David; Rosin, Paul L.; Drage, Nicholas; Richmond, Stephen; Jones, Derek K. 2013. RESDORE: Robust Estimation in Spherical Deconvolution by Outlier Rejection In International Society for Magnetic Resonance in Medicine, 21st Scientific Meeting, Salt Lake City, USA. p. 3148.

Parker GD, Jones DK. (2017). MuSHER: Multi-shell Spherical Harmonic Error Rejection In International Society for Magnetic Resonance in Medicine, 25th Scientific Meeting, Honololu, Hawai'i. p. 3535.

Peled, S., Cory, D. G., Raymond, S. A., Kirschner, D. A., \& Jolesz, F. A. (1999). Water diffusion, T(2), and compartmentation in frog sciatic nerve. Magnetic Resonance in Medicine, 42(5), 911-918.

Pfefferbaum A, Sullivan EV, Adalsteinsson E, Garrick T, Harper C. Postmortem MR imaging of formalin-fixed human brain. Neurolmage. Apr 2004;21(4):1585-1595.

Pierpaoli C. Artifacts in Diffusion MRI, in 'Diffusion MRI: Theory, Methods and Application' (Jones, D.K., Oxford University Press, 2010).

Pajevic S, Pierpaoli C.1999. Color schemes to represent the orientation of anisotropic tissues from diffusion tensor data: application to white matter fiber tract mapping in the human brain. Magn Reson Med. 1999 Sep;42(3):526-40. Erratum in: Magn Reson Med 2000 Jun;43(6):921.

Posse, S., Cuenod, C. A., \& Le Bihan, D. (1993). Human brain: proton diffusion MR spectroscopy. Radiology, 188(3), 719-725. http://doi.org/10.1148/radiology.188.3.8351339

Raffelt, D., Tournier, J. D., Rose, S., Ridgway, G. R., Henderson, R., Crozier, S., et al. (2012). Apparent Fibre Density: A novel measure for the analysis of diffusion-weighted magnetic resonance images. Neurolmage, 59(4), 3976-3994. http://doi.org/10.1016/j.neuroimage.2011.10.045

Rane S, Nair G, Duong TQ. DTI at long diffusion time improves fiber tracking. NMR Biomed. 2010, 23: 459465.

Reese, T. G., Heid, O., Weisskoff, R. M., \& Wedeen, V. J. (2003). Reduction of eddy-current-induced distortion in diffusion MRI using a twice-refocused spin echo. Magnetic Resonance in Medicine, 49(1), 177-182. http://doi.org/10.1002/mrm.10308

Reilly, J. P. (1989). Peripheral nerve stimulation by induced electric currents: Exposure to time-varying magnetic fields. Medical \& Biological Engineering \& Computing, 27(2), 101-110. http://doi.org/10.1007/BF02446217

Reilly, J. P. (1991). Magnetic field excitation of peripheral nerves and the heart: a comparison of thresholds. Medical \& Biological Engineering \& Computing, 29(6), 571-579. http://doi.org/10.1007/BF02446087

Reilly JP. (1995). Nerve Stimulation of Cows and Other Farm Animals by Time-varying Magnetic Fields. Transactions of the ASAE, 38(5), 1487-1494. http://doi.org/10.13031/2013.27974

Reveley C, Seth AK, Pierpaoli C, Silva AC, Yu D, Saunders RC, Leopold DA, Ye FQ. 2015. Superficial white matter fiber systems impede detection of long-range cortical connections in diffusion MR tractography. Proc Natl Acad Sci U S A. 2015 May 26;112(21):E2820-8. doi: 10.1073/pnas.1418198112. Epub 2015 May 11.

Reisert, M., Kellner, E., Dhital, B., Hennig, J., \& Kiselev, V. G. (2017). Disentangling micro from 
mesostructure by diffusion MRI: A Bayesian approach. Neurolmage, 147, 964-975.

http://doi.org/10.1016/j.neuroimage.2016.09.058

Reynaud, O. (2017). Time-Dependent Diffusion MRI in Cancer: Tissue Modeling and Applications. Frontiers in Physics, 5, 581. http://doi.org/10.3389/fphy.2017.00058

Richardson, A. G., McIntyre, C. C., \& Grill, W. M. (2000). Modelling the effects of electric fields on nerve fibres: influence of the myelin sheath. Medical \& Biological Engineering \& Computing, 38(4), 438-446.

Ritchie, J.M. (1995). Physiology of axons. In The Axon (pp. 68-96). Oxford University Press. http://doi.org/10.1093/acprof:oso/9780195082937.003.0004

Ritchie, S. J., Tucker-Drob, E. M., Cox, S. R., Dickie, D. A., Del C Valdés Hernández, M., Corley, J., et al. (2017). Risk and protective factors for structural brain ageing in the eighth decade of life. Brain Structure \& Function, 222(8), 3477-3490. http://doi.org/10.1007/s00429-017-1414-2

Ronen, I., \& Valette, J. (2007). Diffusion-Weighted Magnetic Resonance Spectroscopy (Vol. 131, pp. 733750). Chichester, UK: John Wiley \& Sons, Ltd. http://doi.org/10.1002/9780470034590.emrstm1471

Schaefer, D. J., Bourland, J. D., \& Nyenhuis, J. A. (2000). Review of patient safety in time-varying gradient fields. Journal of Magnetic Resonance Imaging : JMRI, 12(1), 20-29.

Scherrer, B., Schwartzman, A., Taquet, M., Sahin, M., Prabhu, S. P., \& Warfield, S. K. (2016). Characterizing brain tissue by assessment of the distribution of anisotropic microstructural environments in diffusioncompartment imaging (DIAMOND). Magnetic Resonance in Medicine, 76(3), 963-977. http://doi.org/10.1002/mrm.25912

Schneider, T., Brownlee, W., Zhang, H., Ciccarelli, O., Miller, D. H., \& Wheeler-Kingshott, C. (2014). Application of multi-shell NODDI in Multiple Sclerosis. In: (Proceedings) Joint Annual Meeting ISMRMESMRMB. (Pp. 0019-). (2014).

Schilling KG, Janve V, Gao Y, Stepniewska I, Landman BA, Anderson AW. Comparison of 3D orientation distribution functions measured with confocal microscopy and diffusion MRI. Neurolmage 2016; 129: 185-197

Schilling KG, Janve V, Gao Y, Stepniewska I, Landman BA, Anderson AW. Histological validation of diffusion MRI fiber orientation distributions and dispersion. Neurolmage 2018; 165: 200-221.

Sepehrband, F., Alexander, D. C., Kurniawan, N. D., Reutens, D. C., \& Yang, Z. (2016). Towards higher sensitivity and stability of axon diameter estimation with diffusion-weighted MRI. NMR in Biomedicine, 29(3), 293-308. http://doi.org/10.1002/nbm.3462

Setsompop, K., Fan, Q., Stockmann, J., Bilgic, B., Huang, S., Cauley, S. F., et al. (2017). High-resolution in vivo diffusion imaging of the human brain with generalized slice dithered enhanced resolution:

Simultaneous multislice (gSlider-SMS). Magnetic Resonance in Medicine, 57, 167. http://doi.org/10.1002/mrm.26653

Setsompop, K., Ning L., Rathi Y. (2016). A combined Compressed Sensing Super-Resolution Diffusion and gSlider-SMS acquisition/reconstruction for rapid sub-millimeter whole-brain diffusion imaging. Frontiers in Physics, 4. http://doi.org/10.3389/conf.FPHY.2016.01.00009

Setsompop, K., Kimmlingen, R., Eberlein, E., Witzel, T., Cohen-Adad, J., McNab, J. A., et al. (2013). Pushing the limits of in vivo diffusion MRI for the Human Connectome Project. Neurolmage, 80, 220-233. http://doi.org/10.1016/j.neuroimage.2013.05.078

Shepherd, T. M., Thelwall, P. E., Stanisz, G. J., \& Blackband, S. J. (2009). Aldehyde fixative solutions alter the water relaxation and diffusion properties of nervous tissue. Magnetic Resonance in Medicine, 62(1), 26-34. http://doi.org/10.1002/mrm.21977

Shepherd TM, Flint JJ, Thelwall PE, et al. 2009b. Postmortem interval alters the water relaxation and diffusion properties of rat nervous tissue--implications for MRI studies of human autopsy samples. Neurolmage. Feb 1 2009;44(3):820-826.

Sherbondy, A. J., Dougherty, R. F., Ben-Shachar, M., Napel, S., \& Wandell, B. A. (2008). ConTrack: finding the most likely pathways between brain regions using diffusion tractography. Journal of Vision, 8(9), 15.116. http://doi.org/10.1167/8.9.15

Sherbondy, A. J., Rowe, M. C., \& Alexander, D. C. (2010). MicroTrack: an algorithm for concurrent projectome and microstructure estimation. Medical Image Computing and Computer-Assisted Intervention : MICCAI ... International Conference on Medical Image Computing and Computer-Assisted Intervention, 13(Pt 1), 183-190.

Shemesh N, Cohen Y. The effect of experimental parameters on the signal decay in double-PGSE 
experiments: negative diffractions and enhancement of structural information. J Magn Reson. 2008 Dec;195(2):153-61. doi: 10.1016/j.jmr.2008.09.006. Epub 2008 Sep 17.

Shmueli, K., de Zwart, J. A., van Gelderen, P., Li, T.-Q., Dodd, S. J., \& Duyn, J. H. (2009). Magnetic susceptibility mapping of brain tissue in vivo using MRI phase data. Magnetic Resonance in Medicine, 62(6), 1510-1522. http://doi.org/10.1002/mrm.22135

Siow, B., Drobnjak, I., Chatterjee, A., Lythgoe, M. F., \& Alexander, D. C. (2012). Estimation of pore size in a microstructure phantom using the optimised gradient waveform diffusion weighted NMR sequence. Journal of Magnetic Resonance (San Diego, Calif. : 1997), 214(1), 51-60. http://doi.org/10.1016/j.jmr.2011.10.004

Sjölund J, Szczepankiewicz F, Nilsson M, Topgaard D, Westin CF, Knutsson H. 2015. Constrained optimization of gradient waveforms for generalized diffusion encoding. J Magn Reson. 2015 Dec;261:157-68. doi: 10.1016/j.jmr.2015.10.012. Epub 2015 Oct 31.

Slattery, C. F., Zhang, J., Paterson, R. W., Foulkes, A. J. M., Carton, A., Macpherson, K., et al. (2017). ApoE influences regional white-matter axonal density loss in Alzheimer's disease. Neurobiology of Aging, 57, 8-17. http://doi.org/10.1016/j.neurobiolaging.2017.04.021

Sled, J. G., Levesque, I., Santos, A. C., Francis, S. J., Narayanan, S., Brass, S. D., et al. (2004). Regional variations in normal brain shown by quantitative magnetization transfer imaging. Magnetic Resonance in Medicine, 51(2), 299-303. http://doi.org/10.1002/mrm.10701

Sotiropoulos, S. N., Jbabdi, S., Xu, J., Andersson, J. L., Moeller, S., Auerbach, E. J., et al. (2013). Advances in diffusion MRI acquisition and processing in the Human Connectome Project. Neurolmage, 80, 125-143. http://doi.org/10.1016/j.neuroimage.2013.05.057

Stanisz, G. J., Szafer, A., Wright, G. A., \& Henkelman, R. M. (1997). An analytical model of restricted diffusion in bovine optic nerve. Magnetic Resonance in Medicine, 37(1), 103-111.

Stejskal, E. O., \& Tanner, J. E. (1965). Spin Diffusion Measurements: Spin Echoes in the Presence of a Time-Dependent Field Gradient. The Journal of Chemical Physics, 42(1), 288-292. http://doi.org/10.1063/1.1695690

Sullivan, E. V., \& Pfefferbaum, A. (2010). Diffusion Tensor Imaging in Aging and Age-Related Neurodegenerative Disorders. In Diffusion MRI (pp. 624-644). Oxford University Press. http://doi.org/10.1093/med/9780195369779.003.0038

Sun SW, Neil JJ, Liang HF, et al. Formalin fixation alters water diffusion coefficient magnitude but not anisotropy in infarcted brain. Magnetic resonance in medicine. Jun 2005;53(6):1447-1451.

Szczepankiewicz, F., Lasič, S., van Westen, D., Sundgren, P. C., Englund, E., Westin, C. F., et al. (2015). Quantification of microscopic diffusion anisotropy disentangles effects of orientation dispersion from microstructure: Applications in healthy volunteers and in brain tumors. Neurolmage, 104, 241-252. http://doi.org/10.1016/j.neuroimage.2014.09.057

Szczepankiewicz, F., van Westen, D., Englund, E., Westin, C. F., Ståhlberg, F., Lätt, J., et al. (2016). The link between diffusion MRI and tumor heterogeneity: Mapping cell eccentricity and density by diffusional variance decomposition (DIVIDE). Neurolmage, 142, 522-532.

http://doi.org/10.1016/j.neuroimage.2016.07.038

Tam HH, Collins DJ, Brown G, Chau I, Cunningham D, Leach MO, Koh DM. 2013. The role of pre-treatment diffusion-weighted MRI in predicting long-term outcome of colorectal liver metastasis.

Br J Radiol. 2013 Oct;86(1030):20130281. doi: 10.1259/bjr.20130281. Epub 2013 Aug 30.

Tanno, R., Worrall, D. E., Ghosh, A., Kaden, E., Sotiropoulos, S. N., Criminisi, A., \& Alexander, D. C. (2017). Bayesian Image Quality Transfer with CNNs: Exploring Uncertainty in dMRI Super-Resolution. In Medical Image Computing and Computer-Assisted Intervention -- MICCAI 2015 (Vol. 10433, pp. 611619). Cham: Springer International Publishing. http://doi.org/10.1007/978-3-319-66182-7 70

Tanno, Ryutaro \& Ghosh, Aurobrata \& Grussu, Francesco \& Kaden, Enrico \& Criminisi, Antonio \& Alexander, Daniel. (2016). Bayesian Image Quality Transfer. 265-273. 10.1007/978-3-319-46723-8_31.

Tao S, Weavers PT, Trzasko JD, Shu Y, Huston J 3rd, Lee SK, Frigo LM, Bernstein MA. 2017. Gradient preemphasis to counteract first-order concomitant fields on asymmetric MRI gradient systems. Magn Reson Med. 2017 Jun;77(6):2250-2262. doi: 10.1002/mrm.26315. Epub 2016 Jul 4.

Tasaki, I., \& Iwasa, K. (1982a). Further studies of rapid mechanical changes in squid giant axon associated with action potential production. The Japanese Journal of Physiology, 32(4), 505-518.

Tasaki, I., \& Iwasa, K. (1982b). Rapid pressure changes and surface displacements in the squid giant axon 
associated with production of action potentials. The Japanese Journal of Physiology, 32(1), 69-81.

Tax, C. M. W., Jeurissen, B., Vos, S. B., Viergever, M. A., \& Leemans, A. (2014). Recursive calibration of the fiber response function for spherical deconvolution of diffusion MRI data. Neurolmage, 86, 67-80. http://doi.org/10.1016/j.neuroimage.2013.07.067

Tax C.M.W., Vos S.B., Leemans A. (2016) Checking and Correcting DTI Data. In: Van Hecke W., Emsell L., Sunaert S. (eds) Diffusion Tensor Imaging. Springer, New York, NY

Tax CM, Otte WM, Viergever MA, Dijkhuizen RM, Leemans A. 2015. REKINDLE: robust extraction of kurtosis INDices with linear estimation. Magn Reson Med. 2015 Feb;73(2):794-808. doi: 10.1002/mrm.25165. Epub 2014 Mar 31.

Tax CM, Rudrapatna RS, Witzel T, Jones DK. 2017. Disentangling in two dimensions in the living human brain: Feasbility of relaxometry-diffusometry using ultra-strong gradients, International Society Magnetic Resonance in Medicine, 2017, p. 838

Tax CMW , de Almeida Martins JP , Szczepankiewicz F, Westin C-F , Chamberland M , Topgaard D, and Jones DK. From physical chemistry to human brain biology: unconstrained inversion of 5-dimensional diffusion-T2 correlation data. International Society Magnetic Resonance in Medicine, 2018, p. 1101

Topgaard D, Söderman O. 20102. Self-Diffusion in Two- and Three-Dimensional Powders of Anisotropic Domains: An NMR Study of the Diffusion of Water in Cellulose and Starch J. Phys. Chem. B, 2002, 106 (46), pp 11887-11892

Tournier JD, Calamante F, Gadian DG, Connelly A. 2004. Direct estimation of the fiber orientation density function from diffusion-weighted MRI data using spherical deconvolution.

Neuroimage. 2004 Nov;23(3):1176-85.

Tournier JD, Calamante F, Connelly A. 2007. Robust determination of the fibre orientation distribution in diffusion MRI: non-negativity constrained super-resolved spherical deconvolution. Neuroimage. 2007 May 1;35(4):1459-72. Epub 2007 Feb 21.

Turner, R. (1998). Diffusion Imaging with Echo-Planar Imaging. In Echo-Planar Imaging (pp. 311-324). Berlin, Heidelberg: Springer Berlin Heidelberg. http://doi.org/10.1007/978-3-642-80443-4_9

Turner, R. (2001). BOLD localization: the implications of vascular architecture. Neurolmage, 13(6), 1011. http://doi.org/10.1016/S1053-8119(01)92347-X

Turner, R. (2002). How much cortex can a vein drain? Downstream dilution of activation-related cerebral blood oxygenation changes. Neurolmage, 16(4), 1062-1067.

Turner, R., Le Bihan, D., Maier, J., Vavrek, R., Hedges, L. K., \& Pekar, J. (1990). Echo-planar imaging of intravoxel incoherent motion. Radiology, 177(2), 407-414.

http://doi.org/10.1148/radiology.177.2.2217777

Upadhyay, J., Hallock, K., Ducros, M., Kim, D.-S., \& Ronen, I. (2008). Diffusion tensor spectroscopy and imaging of the arcuate fasciculus. Neurolmage, 39(1), 1-9.

http://doi.org/10.1016/j.neuroimage.2007.08.046

Valette, J., Ligneul, C., Marchadour, C., Najac, C., \& Palombo, M. (2018). Brain metabolite diffusion from ultra-short to ultra-long time scales: what do we learn, where should we go? Frontiers in neuroscience, 12, 2. https://doi.org/10.3389/fnins.2018.00002.

van der Toorn, A., Dijkhuizen, R. M., Tulleken, C. A., \& Nicolay, K. (1996). Diffusion of metabolites in normal and ischemic rat brain measured by localized 1H MRS. Magnetic Resonance in Medicine, 36(6), 914922.

van Dusschoten, D., Moonen, C. T., de Jager, P. A., \& Van As, H. (1996). Unraveling diffusion constants in biological tissue by combining Carr-Purcell-Meiboom-Gill imaging and pulsed field gradient NMR. Magnetic Resonance in Medicine, 36(6), 907-913.

Van Steenkiste, G., Jeurissen, B., Veraart, J., Dekker, den, A. J., Parizel, P. M., Poot, D. H. J., \& Sijbers, J. (2016). Super-resolution reconstruction of diffusion parameters from diffusion-weighted images with different slice orientations. Magnetic Resonance in Medicine, 75(1), 181-195. http://doi.org/10.1002/mrm.25597

Venkataramanan, L., Yi-Qiao Song, \& Hurlimann, M. D. (2002). Solving Fredholm integrals of the first kind with tensor product structure in 2 and 2.5 dimensions. IEEE Transactions on Signal Processing, 50(5), 1017-1026. http://doi.org/10.1109/78.995059

Veraart, J., Novikov, D. S., \& Fieremans, E. (2017). TE dependent Diffusion Imaging (TEdDI) distinguishes between compartmental $\mathrm{T} 2$ relaxation times. Neurolmage. 
http://doi.org/10.1016/j.neuroimage.2017.09.030

Veraart, J., Rajan, J., Peeters, R. R., Leemans, A., Sunaert, S., \& Sijbers, J. (2012). Comprehensive framework for accurate diffusion MRI parameter estimation. Magnetic Resonance in Medicine, 70(4), 972-984. http://doi.org/10.1002/mrm.24529

Veraart, J., Sijbers, J., Sunaert, S., Leemans, A., \& Jeurissen, B. (2013). Weighted linear least squares estimation of diffusion MRI parameters: strengths, limitations, and pitfalls. Neurolmage, 81, 335-346. http://doi.org/10.1016/j.neuroimage.2013.05.028

Vigneau-Roy, N., Bernier, M., Descoteaux, M., \& Whittingstall, K. (2013). Regional variations in vascular density correlate with resting-state and task-evoked blood oxygen level-dependent signal amplitude. Human Brain Mapping, 35(5), 1906-1920. http://doi.org/10.1002/hbm.22301

Weavers PT, Tao S, Trzasko JD, Frigo LM, Shu Y, Frick MA, Lee, Foo TK, Bernstein MA. 2018. B0 concomitant field compensation for MRI systems employing asymmetric transverse gradient coils. Magn Reson Med. 2018 Mar;79(3):1538-1544. doi: 10.1002/mrm.26790. Epub 2017 Jun 21.

Westin, C. F., Knutsson, H., Pasternak, O., Szczepankiewicz, F., Özarslan, E., van Westen, D., et al. (2016). Qspace trajectory imaging for multidimensional diffusion MRI of the human brain. Neurolmage, 135, 345-362. http://doi.org/10.1016/i.neuroimage.2016.02.039

Wetscherek A, Stieltjes B, Laun FB. 2015. Flow-compensated intravoxel incoherent motion diffusion imaging. Magn Reson Med. 2015 Aug;74(2):410-9. doi: 10.1002/mrm.25410. Epub 2014 Aug 12.

White, T., \& Lim, K. O. (2010). Diffusion MRI in Psychiatric Disorders. In Diffusion MRI (pp. 608-623). Oxford University Press. http://doi.org/10.1093/med/9780195369779.003.0037

Williams, R. J., Reutens, D. C., \& Hocking, J. (2016). Influence of BOLD Contributions to Diffusion fMRI Activation of the Visual Cortex. Frontiers in Neuroscience, 10(277), 279. http://doi.org/10.3389/fnins.2016.00279

Wilm, B. J., Barmet, C., Gross, S., Kasper, L., Vannesjo, S. J., Haeberlin, M., et al. (2017). Single-shot spiral imaging enabled by an expanded encoding model: Demonstration in diffusion MRI. Magnetic Resonance in Medicine, 77(1), 83-91. http://doi.org/10.1002/mrm.26493

Wilm, B. J., Nagy, Z., Barmet, C., Vannesjo, S. J., Kasper, L., Haeberlin, M., et al. (2015). Diffusion MRI with concurrent magnetic field monitoring. Magnetic Resonance in Medicine, 74(4), 925-933. http://doi.org/10.1002/mrm.25827

Wolff, S. D., \& Balaban, R. S. (1989). Magnetization transfer contrast (MTC) and tissue water proton relaxation in vivo. Magnetic Resonance in Medicine, 10(1), 135-144.

Wong, E. C., Cox, R. W., \& Song, A. W. (1995). Optimized isotropic diffusion weighting. Magnetic Resonance in Medicine, 34(2), 139-143. http://doi.org/10.1002/mrm.1910340202

Wood, E. T., Ercan, A. E., Branzoli, F., Webb, A., Sati, P., Reich, D. S., \& Ronen, I. (2015). Reproducibility and optimization of in vivohuman diffusion-weighted MRS of the corpus callosum at 3T and 7T. NMR in Biomedicine, 28(8), 976-987. http://doi.org/10.1002/nbm.3340

Wood, E. T., Ercan, E., Sati, P., Cortese, I. C. M., Ronen, I., \& Reich, D. S. (2017). Longitudinal MR spectroscopy of neurodegeneration in multiple sclerosis with diffusion of the intra-axonal constituent $\mathrm{N}$-acetylaspartate. Neurolmage. Clinical, 15, 780-788. http://doi.org/10.1016/j.nicl.2017.06.028

Wood, E. T., Ronen, I., Techawiboonwong, A., Jones, C. K., Barker, P. B., Calabresi, P., et al. (2012). Investigating axonal damage in multiple sclerosis by diffusion tensor spectroscopy. The Journal of Neuroscience : the Official Journal of the Society for Neuroscience, 32(19), 6665-6669. http://doi.org/10.1523/JNEUROSCI.0044-12.2012

Xu, J., Does, M. D., \& Gore, J. C. (2009). Sensitivity of MR diffusion measurements to variations in intracellular structure: effects of nuclear size. Magnetic Resonance in Medicine, 61(4), 828-833. http://doi.org/10.1002/mrm.21793

Xu, J., Li, H., Harkins, K. D., Jiang, X., Xie, J., Kang, H., et al. (2014). Mapping mean axon diameter and axonal volume fraction by MRI using temporal diffusion spectroscopy. Neurolmage, 103, 10-19. http://doi.org/10.1016/j.neuroimage.2014.09.006

Xu, J., Li, H., Li, K., Harkins, K. D., Jiang, X., Xie, J., et al. (2016). Fast and simplified mapping of mean axon diameter using temporal diffusion spectroscopy. NMR in Biomedicine, 29(4), 400-410.

Yablonskiy, D. A., \& Sukstanskii, A. L. (2010). Theoretical models of the diffusion weighted MR signal. NMR in Biomedicine, 23(7), 661-681. http://doi.org/10.1002/nbm.1520

Yuan J, Shen GX. 2007. Gradient coil design using Bi-2223 high temperature superconducting tape for 
magnetic resonance imaging. Med Eng Phys. 2007 May;29(4):442-8. Epub 2006 Jul 27.

Zhang, Hui, Dyrby, T. B., \& Alexander, D. C. (2011a). Axon diameter mapping in crossing fibers with diffusion MRI. Medical Image Computing and Computer-Assisted Intervention : MICCAI ... International

Conference on Medical Image Computing and Computer-Assisted Intervention, 14(Pt 2), 82-89.

Zhang, H, Hubbard, P. L., Parker, G. J. M., \& Alexander, D. C. (2011b). Axon diameter mapping in the presence of orientation dispersion with diffusion MRI. Neurolmage, 56(3), 1301-1315.

http://doi.org/10.1016/j.neuroimage.2011.01.084

Zhang, H, Schneider, T., Wheeler-Kingshott, C. A., \& Alexander, D. C. (2012). NODDI: Practical in vivo neurite orientation dispersion and density imaging of the human brain. Neurolmage, 61(4), 1000-1016. http://doi.org/10.1016/j.neuroimage.2012.03.072

Zheng, D. D., Liu, Z. H., Fang, J., Wang, X. Y., \& Zhang, J. (2012). The effect of age and cerebral ischemia on diffusion-weighted proton MR spectroscopy of the human brain. American Journal of Neuroradiology, 33(3), 563-568. http://doi.org/10.3174/ajnr.A2793

Zielinski, B. A., Prigge, M. B. D., Nielsen, J. A., Froehlich, A. L., Abildskov, T. J., Anderson, J. S., et al. (2014). Longitudinal changes in cortical thickness in autism and typical development. Brain : a Journal of Neurology, 137(6), 1799-1812. http://doi.org/10.1093/brain/awu083 


\section{FIGURE02}

Click here to download 9. Figure: Figure02.pdf

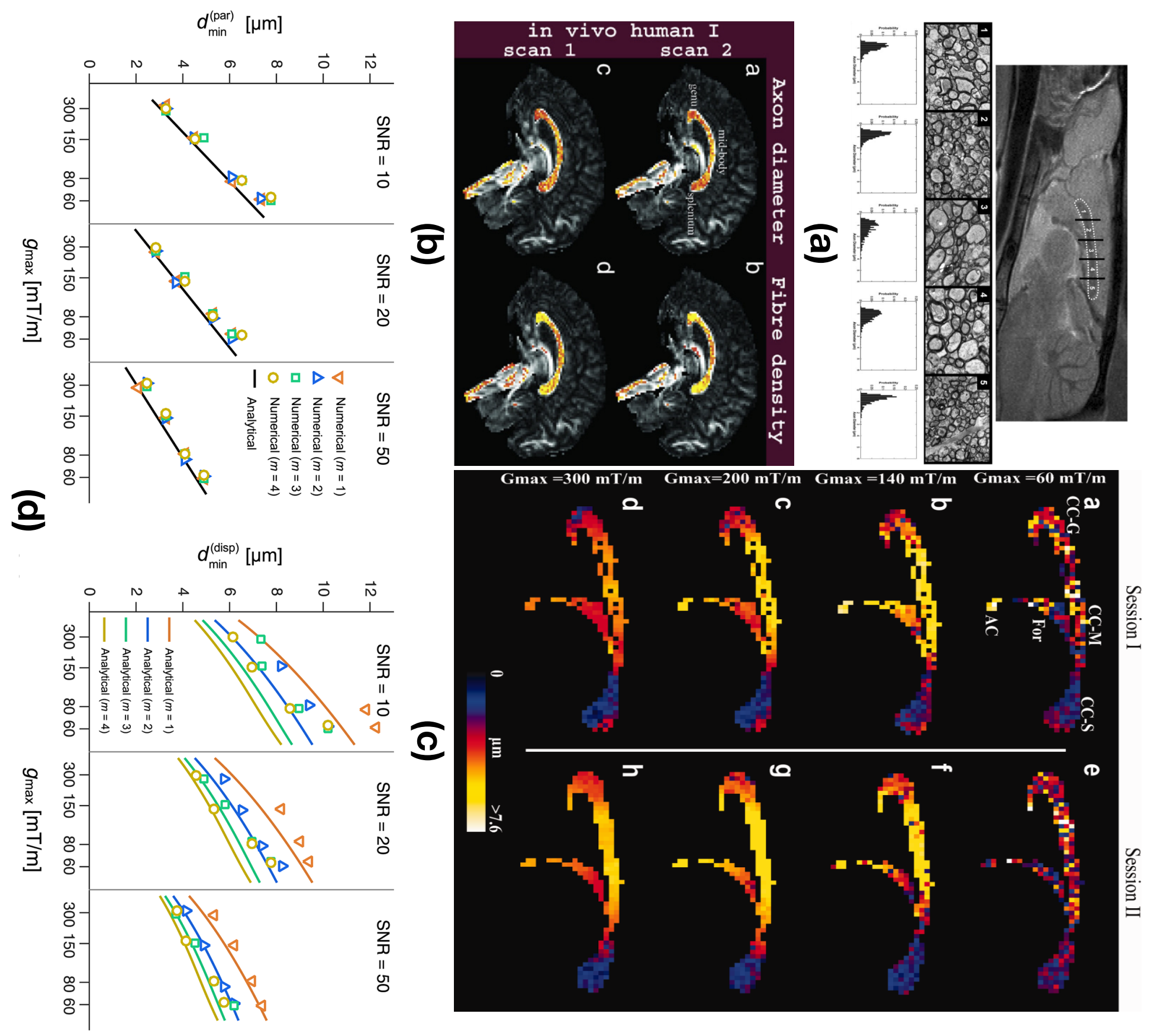



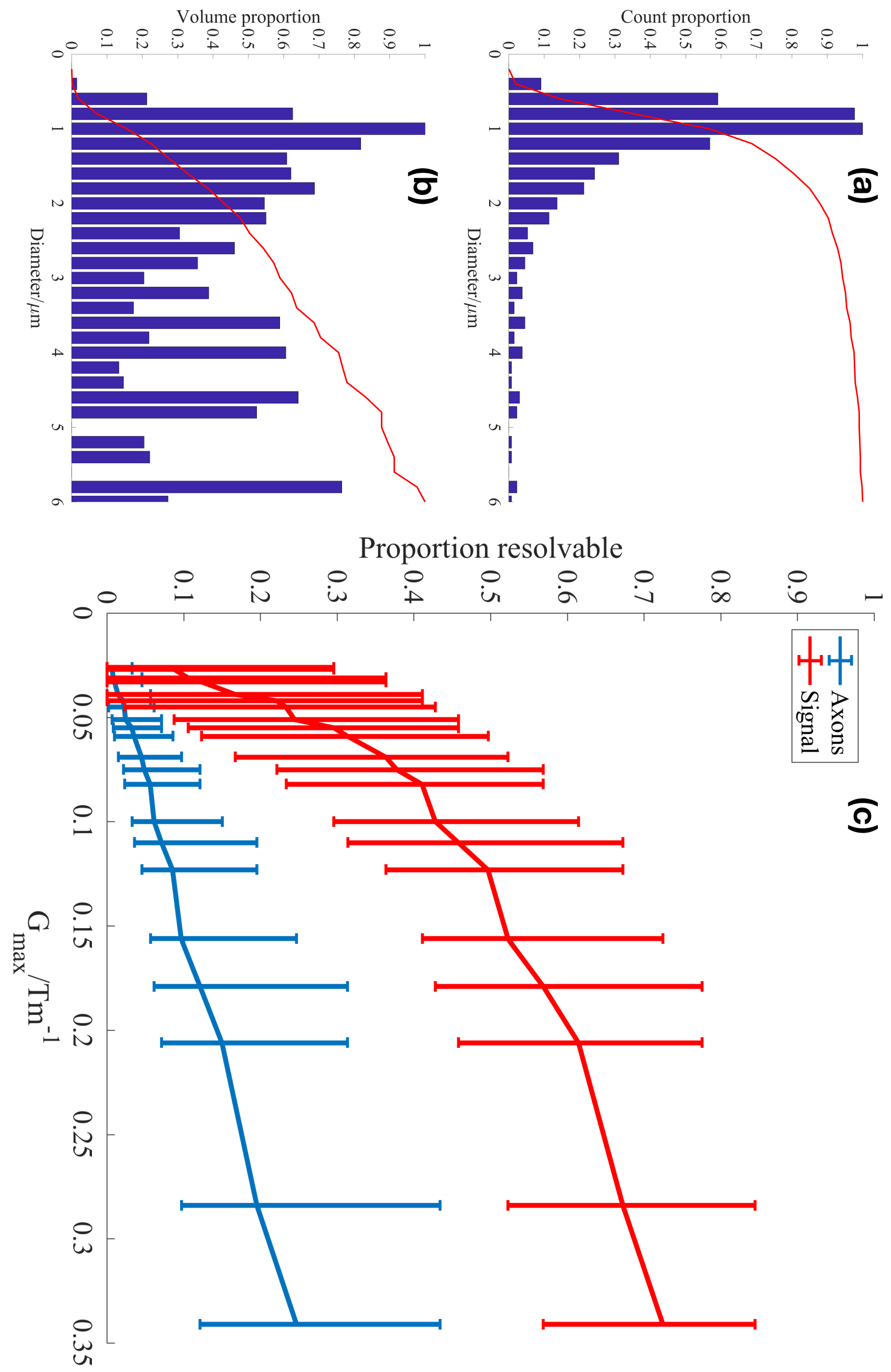


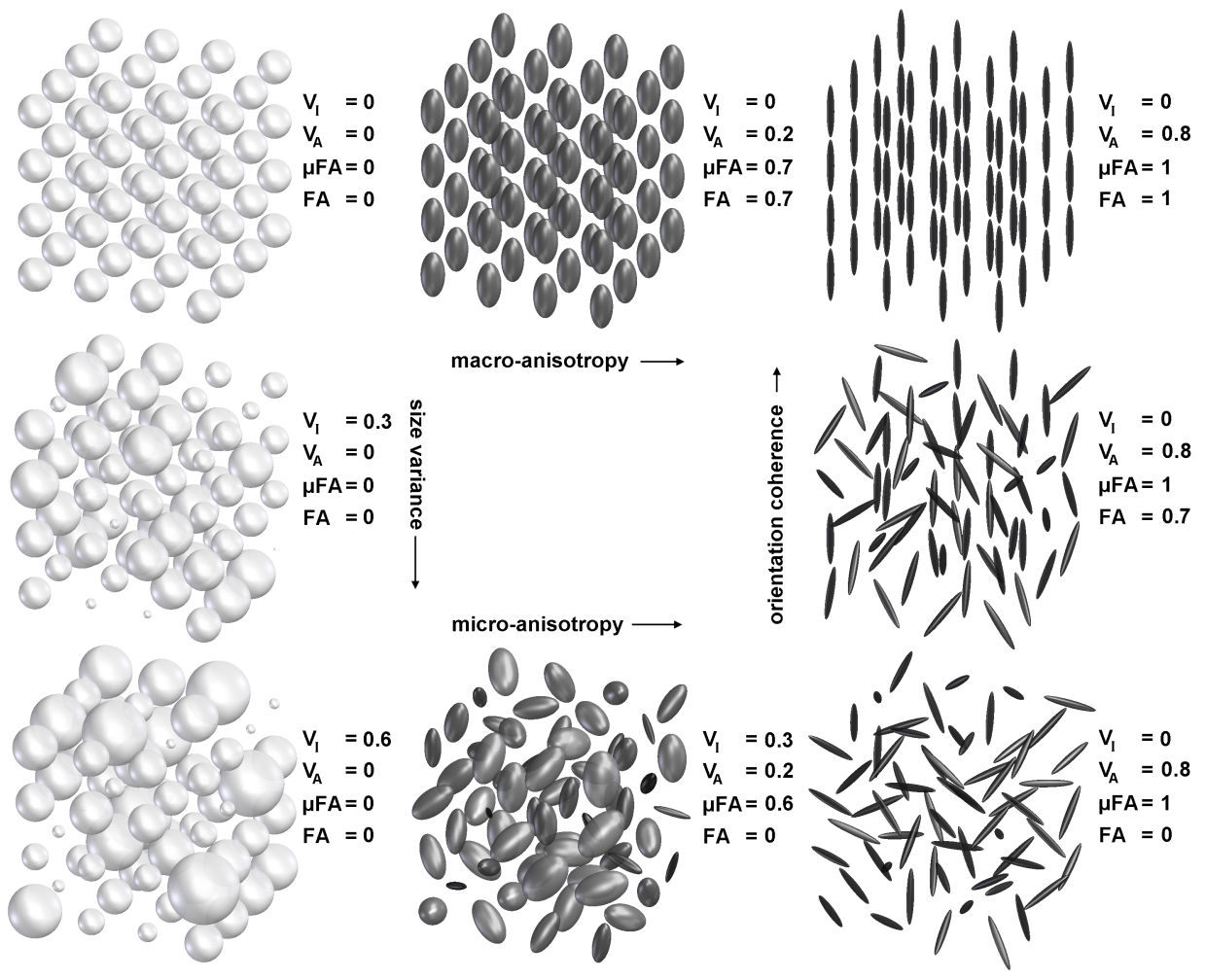


Click here to download 9. Figure: Figure04b.pdf

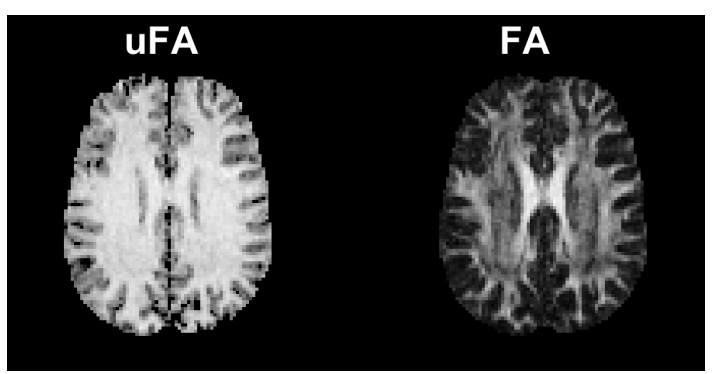


FIGURE06

Click here to download 9. Figure: Figure06.pdf

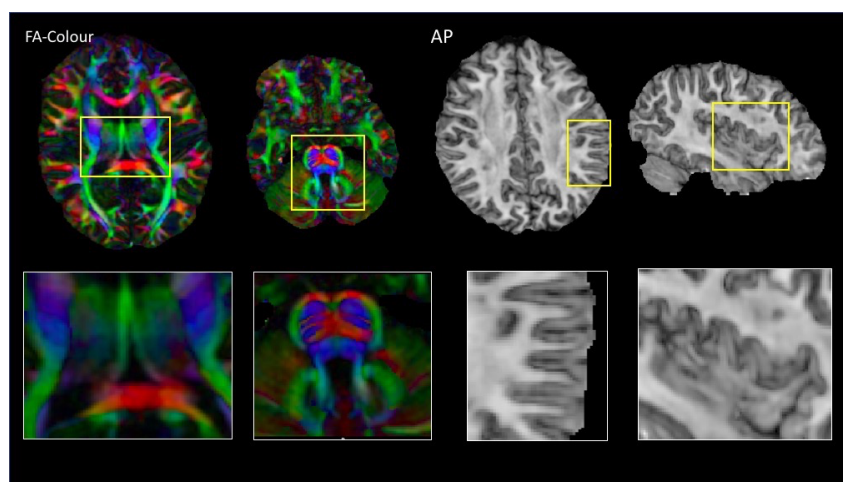



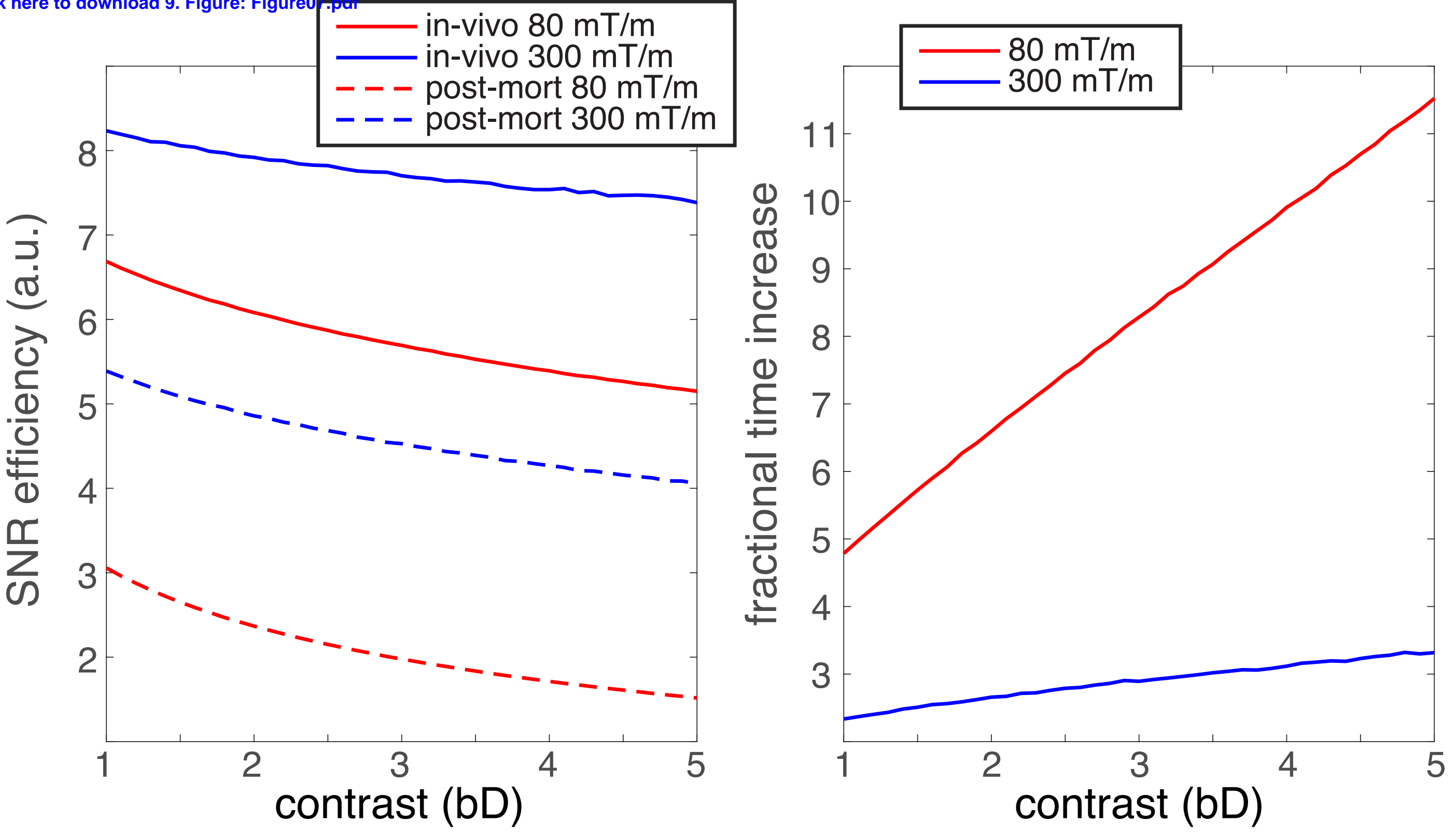

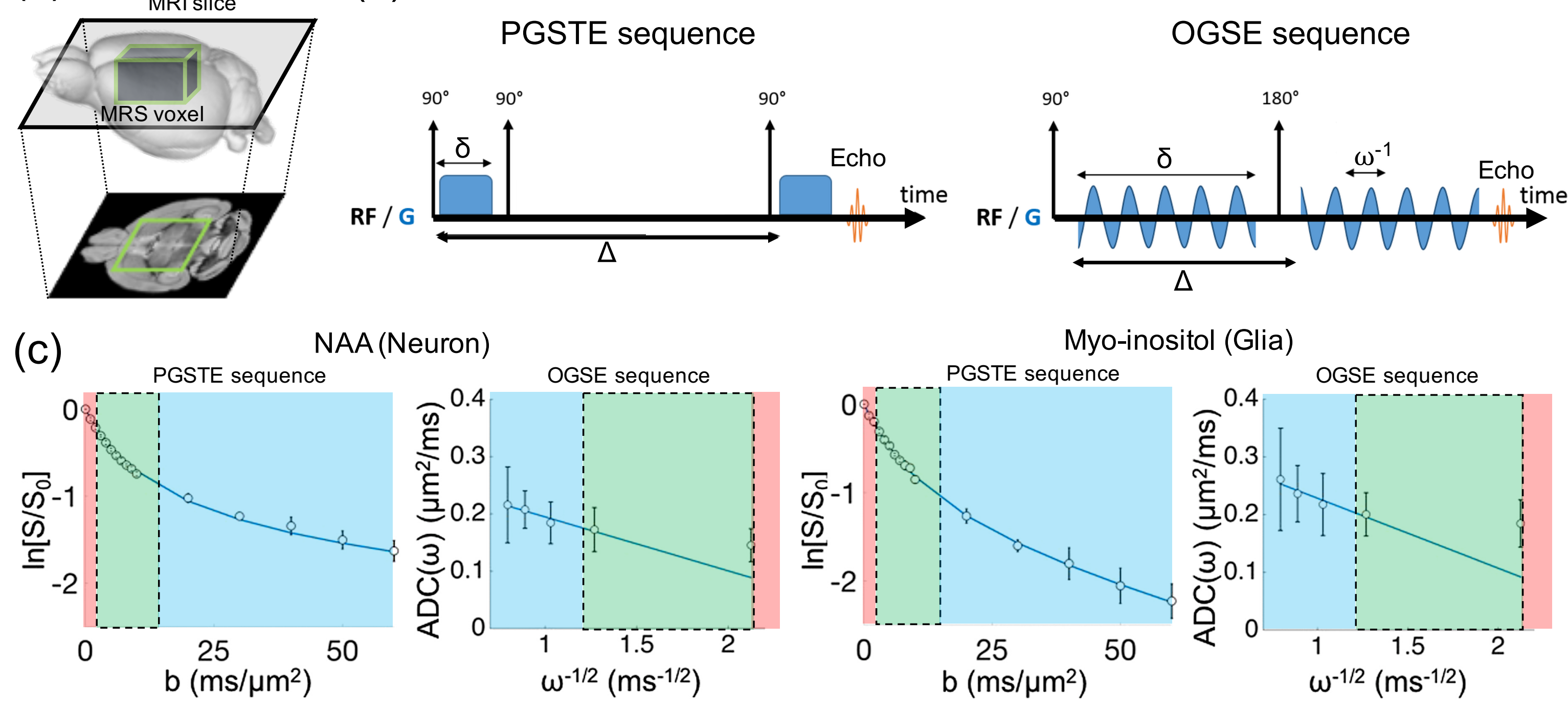

Myo-inositol (Glia)

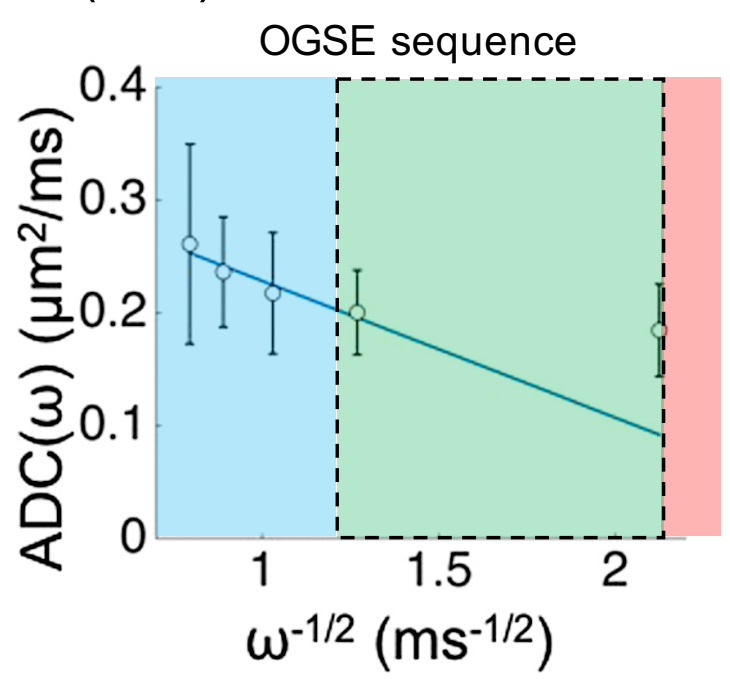

Clinical scanner $\left(G_{\max }=80 \mathrm{mT} / \mathrm{m}\right) \quad$ Preclinical scanner $\left(G_{\max }=800 \mathrm{mT} / \mathrm{m}\right)$ 
Click here to download 9. Figure: Figure10.pdf
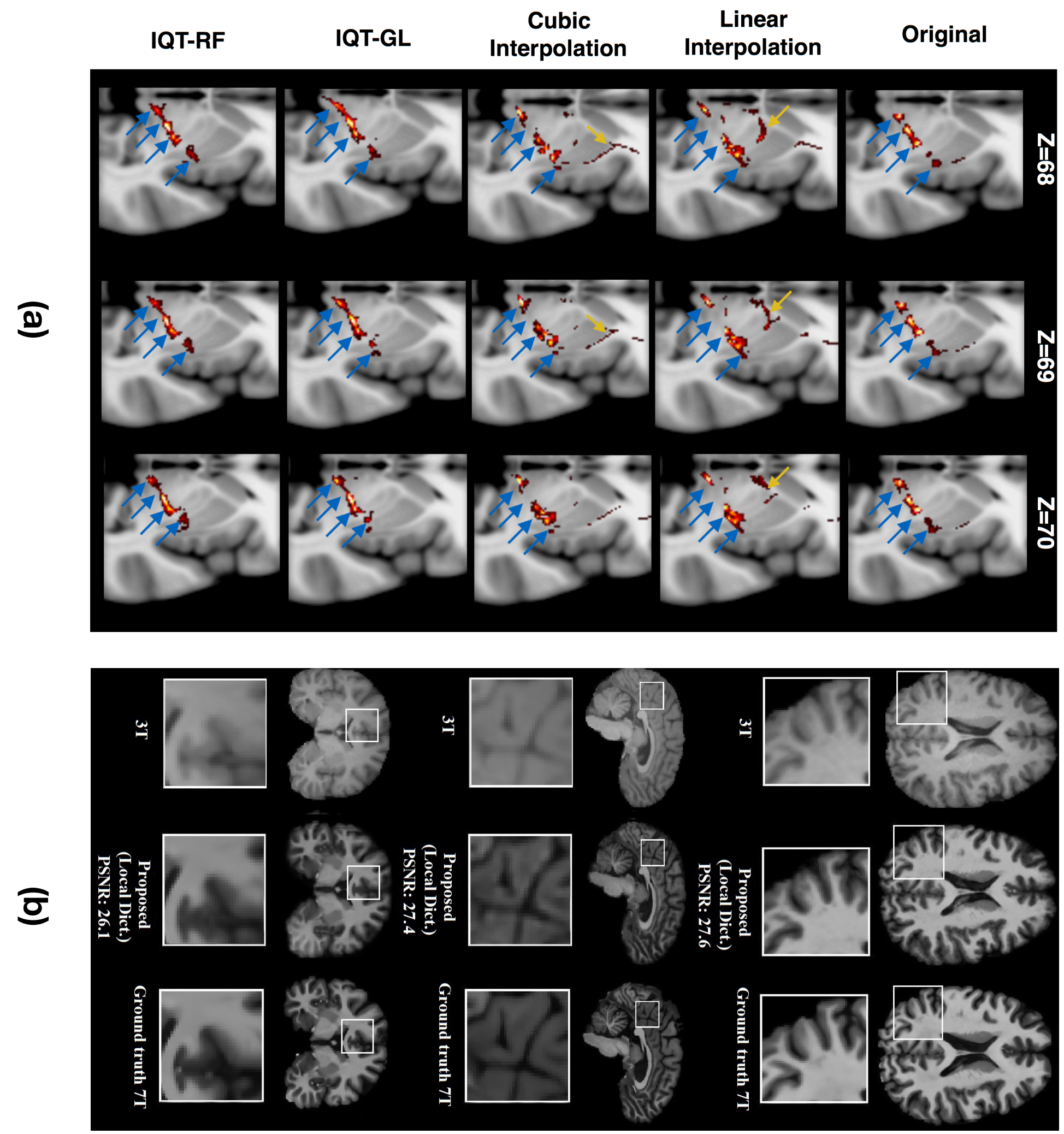


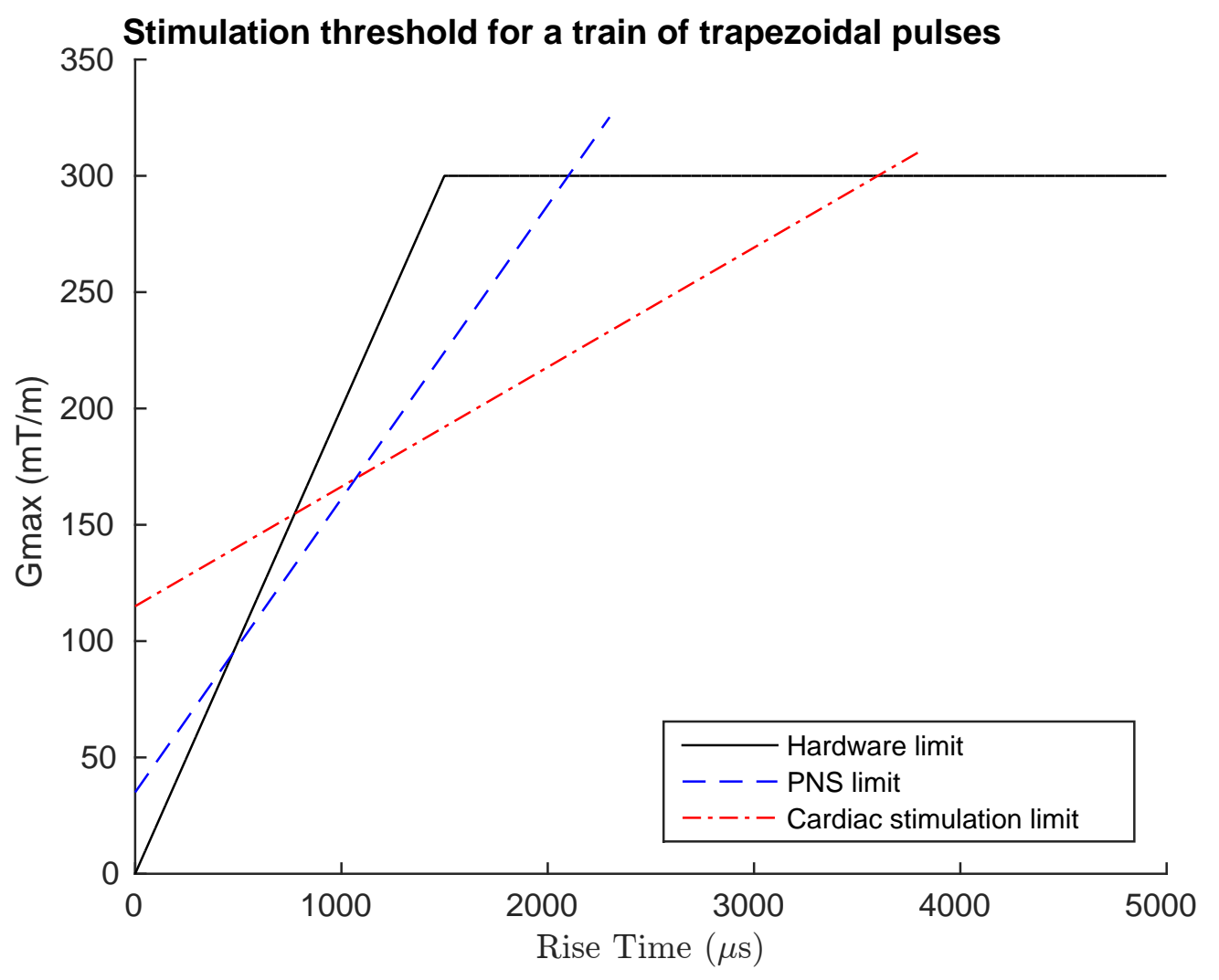

